Issued by Sandia National Laboratories, operated for the United States Department of Energy by Sandia Corporation.

NOTICE: This report was prepared as an account of work sponsored by agency of the United States Government. Neither the United States Governinent nor any agency thereof, nor any of their employecss, nor any of the contractors, subcontractors, or their employees, makes any ivarranty, express or implied, or assumes ary ierjal liability or responsibility for the accuracy, completeness, or isefininess of any information, apparatus, product, or process disclose $0^{\prime}$, or represenis that its use would not infringe privately owned ights. Reference herein to any specific commercial product, process, or service by trade name, trademark, manufacturer, or otherwise, does not necessarily constitute or imply its endorsement, recommendation, or favoring by the United States Government, any agency thereof or any of their contractors or subconractors. The views and opinions expressed herein do not necessarily state or reflect those of the United States Government, any agency thereoi or any of their contractors or subcontractors.

This report has been reproduced from the best available copy.

Available to DOE and DOE contractors from:

Office of Scientific and Technical Information

P.O. Box 62

Oak Ridge TN 37831

Prices available from (615) 576-8401, FTS 626-8401.

Available to the public from:

National Technical Information Service

U.S. Department of Commerce

5285 Port Royal Rd.

Springfield, VA 22161. 
SAND91-8003

DE92 003587

Unlimited Release

Printed August 1991

\title{
SPIN (Version 3.83)*: A FORTRAN PROGRAM FOR MODELING ONE-DIMENSIONAL ROTATING-DISK/STAGNATION-FLOW CHEMICAL VAPOR DEPOSITION REACTORS
}

\author{
Michael E. Coltrin \\ Surface Processing Sciences Division \\ Sandia National Laboratories \\ Albuquerque, NM 87185 \\ Robert J. Kee, Gregory H. Evans, Ellen Meeks, \\ Fran M. Rupley, and Joseph F. Grcar \\ Computational Mechanics Division \\ Sandia National Laboratories \\ Livermore, CA 94551
}

\begin{abstract}
This report documents the SPIN Fortran computer program that computes species, temperature and velocity profiles, and deposition rates in a steady-state one-dimensional rotating disk or stagnation-point flow chemical vapor deposition (CVD) reactor. The program accounts for finite-rate gas-phase and surface chemical kinetics and multicomponent molecular transport. The governing differential equations form a twopoint boundary value problem. After discretization by a finite difference procedure, the resulting nonlinear algebraic equations are solved by a modified Newton algorithm. The Newton algorithm is implemented in a software package called TWOPNT. SPIN also runs in conjunction with the CHEMKIN, SURFACE CHEMKIN, and TRANSPORT software packages that facilitate definition of the gas-phase and surface chemical reaction mechanisms and the transport properties. Discussion of an example problem illustrates many of the program's capabilities.
\end{abstract}

* This document describes the features in version 3.83. We expect that this software package will continue to evolve, and thus later versions may render portions of this document out of date. 


\section{CONTENTS}

NOMENCLATURE

$\frac{\text { Page }}{5}$

I. INTRODUCTION

II. ROTATING-DISK/STAGNATION-POINT EQUATIONS

7

III. NUMERICAL SOLUTION METHOD

Finite Difference Approximations $\quad 13$

$\begin{array}{ll}\text { Starting Estimates } & 14\end{array}$

Continuation Start-up Procedure \& User-Specified Temperature Profile $\quad 15$

Modified Damped Newton's Method $\quad 16$

$\begin{array}{ll}\text { Jacobian Matrix } & 17\end{array}$

Adaptation $\quad 17$

$\begin{array}{lr}\text { Time Stepping } & 18\end{array}$

$\begin{array}{lr}\text { Sensitivity Analysis } & 19\end{array}$

IV. PROGRAM STRUCTURE $\quad 20$

User-Supplied Program $\quad 22$

V. PROGRAM INPUT AND OUTPUT $\quad 22$

Keyword Descriptions $\quad 24$

Restart and Save Files $\quad 34$

$\begin{array}{ll}\text { VI. SAMPLE PROBLEM } & 40\end{array}$

Input to CHEMKIN Interpreter for the Example $\quad 42$

Output from CHEMKIN Interpreter for the Example $\quad 44$

Input to SURFACE CHEMKIN Input for the Example $\quad 46$

Output from SURFACE CHEMKIN Input for the Example $\quad 47$

Input to SPIN for the Example $\quad 48$

$\begin{array}{ll}\text { Output from SPIN for the Example } & 49\end{array}$

$\begin{array}{ll}\text { REFERENCES } & 59\end{array}$ 


\section{NOMENCLATURE}

$a_{i} \quad$ Scaled radial velocity (V) at the inlet

$a_{k} \quad$ Activity of $k$ th bulk-phase species

$A_{i} \quad$ Pre-exponential factor in the rate constant of the $i$ th reaction

$c_{p} \quad$ Specific heat at constant pressure for the gas mixture

$c_{p_{k}} \quad$ Specific heat at constant pressure of the $k$ th species

$C_{p_{k}}^{o} \quad$ Standard state specific heat at constant pressure of the $k$ th species

$D_{k m} \quad$ Mixture-averaged diffusion coefficient

$D_{k}^{T} \quad$ Thermal diffusion coefficient

$\mathcal{D}_{j k} \quad$ Binary diffusion coefficient between species $j$ and $k$

$E_{A} \quad$ Activation Energy

$G \quad$ Bulk growth rate

$h_{k} \quad$ Specific enthalpy of the $k$ th species

$h_{m} \quad$ Heat transfer coefficient

$i \quad$ Reaction index

$I \quad$ Total number of reactions

$J \quad$ Total number of grid nodes

$j_{k} \quad$ Flux of gas-phase species $k$ into the surface

$k \quad$ Species index

$K \quad$ Total number of species

$K_{b} \quad$ Total number of bulk species

$K_{b}^{f} \quad$ Index of the first bulk-phase species in the first bulk phase

$K_{b}^{l} \quad$ Index of the last bulk-phase species in the last bulk phase

$K_{g} \quad$ Total number of gas-phase species

$K_{g}^{f} \quad$ Index of the first gas-phase species

$K_{g}^{l} \quad$ Index of the last gas-phase species

$K_{s} \quad$ Total number of surface species

$K_{s}^{f} \quad$ Index of the first surface-phase species in the first surface phase

$K_{s}^{l} \quad$ Index of the last surface-phase species in the last surface phase

$K_{\text {phase }}$ Vector containing the number of species in each phase

$K_{c_{i}} \quad$ Equilibrium constant in concentration units for the $i$ th reaction

$K_{p_{i}} \quad$ Equilibrium constant in pressure units for the $i$ th reaction

$M \quad$ Number of coefficients in polynomial fits to $C_{p}^{o} / R$

$\bar{M} \quad$ Mean molecular weight of a mixture

$M_{k} \quad$ Molecular weight of $k$ th species

$N_{b} \quad$ Total number of bulk phases

$N_{b}^{f} \quad$ Index of the first bulk phase

$N_{b}^{l} \quad$ Index of the last bulk phase

$N_{s} \quad$ Total number of surface phases

$N_{s}^{f} \quad$ Index of the first surface phase

\section{CGS Units}

$1 / \sec$

none

depends on reaction

ergs/(g K)

ergs $/(\mathrm{g} \mathrm{K})$

ergs/(mole $\mathrm{K})$

$\mathrm{cm}^{2} / \mathrm{sec}$

$\mathrm{g} /(\mathrm{cm}-\mathrm{sec})$

$\mathrm{cm}^{2} / \mathrm{sec}$

ergs/mole

$\mathrm{cm} / \mathrm{sec}$

ergs/g

$\mathrm{gm} /\left(\sec ^{3} \mathrm{~K}\right)$

mole $/\left(\mathrm{cm}^{2}-\mathrm{sec}\right)$

depends on reaction depends on reaction

$\mathrm{g} /$ mole

$\mathrm{g} / \mathrm{mole}$ 


\begin{tabular}{|c|c|c|}
\hline$N_{s}^{l}$ & Index of the last surface phase & \\
\hline$o_{i}$ & Scaled circumferential velocity $(\mathrm{W})$ at the inlet & $1 / \mathrm{sec}$ \\
\hline$P$ & Pressure & dynes $/ \mathrm{cm}^{2}$ \\
\hline$\dot{P}$ & Energy source term for heating of surface & $\mathrm{ergs} /\left(\mathrm{cm}^{2}-\mathrm{sec}\right)$ \\
\hline$p_{m}$ & Spatially-varying component of pressure appearing in radial momentum equation & dynes $/ \mathrm{cm}^{2}$ \\
\hline$Q$ & Net Heat transfer from reactor to ambient & ergs/sec \\
\hline$\dot{q}$ & Total energy deposited into the gas from a heat source & ergs $/\left(\mathrm{cm}^{2}\right.$-sec $)$ \\
\hline$q_{i}$ & Rate of progress of the $i$ th gas-phase reaction & moles $/\left(\mathrm{cm}^{3} \mathrm{sec}\right)$ \\
\hline$R$ & Universal gas constant & ergs/(mole K) \\
\hline$r$ & Radial coordinate & $\mathrm{cm}$ \\
\hline$\dot{s}_{k}$ & Production rate of the $k$ th species due to surface reactions & moles $/\left(\mathrm{cm}^{2} \mathrm{sec}\right)$ \\
\hline$S_{q}$ & Spatially distributed thermal energy source & $\mathrm{erg} /\left(\mathrm{cm}^{3} \mathrm{sec}\right)$ \\
\hline$t$ & Time & $\sec$ \\
\hline$T$ & Temperature & $\mathrm{K}$ \\
\hline$T_{o}$ & Ambient temperature & $\mathrm{K}$ \\
\hline$T_{\text {surf }}$ & Surface temperature & $\mathrm{K}$ \\
\hline$T_{w}$ & Temperature to which the surface radiates & $\mathrm{K}$ \\
\hline$u$ & Axial velocity & $\mathrm{cm} / \mathrm{sec}$ \\
\hline$V$ & Scaled radial velocity & $1 / \sec$ \\
\hline$V$ & Volume & $\mathrm{cm}^{3}$ \\
\hline$V_{c}$ & Correction velocity & $\mathrm{cm} / \mathrm{sec}$ \\
\hline$V_{k}$ & Diffusion velocity of $k$ th species & $\mathrm{cm} / \mathrm{sec}$ \\
\hline$W$ & Scaled circumferential velocity & $1 / \sec$ \\
\hline$W_{k}$ & Molecular weight of $k$ th species & $\mathrm{g} / \mathrm{mole}$ \\
\hline$w_{s}$ & Half-width of the distribution of energy source term & $\mathrm{cm}$ \\
\hline$X_{k}$ & Mole fraction of the $k$ th species & none \\
\hline$x$ & Axial coordinate & none \\
\hline$\left[X_{k}\right]$ & Molar concentration of $k$ th species & moles $/ \mathrm{cm}^{3}$ \\
\hline$x_{s}$ & Position of the center of the energy source term & $\mathrm{cm}$ \\
\hline$Y_{k}$ & Mass fraction of the $k$ th species & none \\
\hline$Z_{k}$ & Site fractions of the $k$ th species on a site & none \\
\hline \multicolumn{3}{|c|}{ GREEK } \\
\hline$\lambda$ & Thermal conductivity & $\mathrm{erg} /(\mathrm{cm} \mathrm{K} \mathrm{sec})$ \\
\hline$\rho$ & Mass density & $\mathrm{g} / \mathrm{cm}^{3}$ \\
\hline$\nu_{k i}$ & Stoichiometric coefficients of the $k$ th reaction, $\nu_{k i}=\nu_{k i}^{\prime \prime}-\nu_{k i}^{\prime}$ & \\
\hline$\nu_{k i}^{\prime}$ & Stoichiometric coefficients of the $k$ th reactant species in the $i$ th reaction & \\
\hline$\nu_{k i}^{\prime \prime}$ & Stoichiometric coefficients of the $k$ th product species in the $i$ th reaction & \\
\hline $\begin{array}{l}\dot{\omega}_{k} \\
\sigma_{k}\end{array}$ & $\begin{array}{l}\text { Chemical production rate of the } k \text { th species due to gas-phase reactions } \\
\text { Number of surface sites a surface species occupies }\end{array}$ & moles $/\left(\mathrm{cm}^{3} \mathrm{sec}\right.$ \\
\hline$\tau$ & Nominal residence time & $\sec$ \\
\hline
\end{tabular}




\section{SPIN (Version 3.83): A FORTRAN PROGRAM FOR MODELING ONE-DIMENSIONAL ROTATING-DISK/STAGNATION-FLOW CHEMICAL VAPOR DEPOSITION REACTORS}

\section{INTRODUCTION}

In a rotating-disk reactor a heated substrate spins (at typical speeds of $1000 \mathrm{rpm}$ or more) in an enclosure through which the reactants flow. The rotating disk geometry has the important property that in certain operating regimes ${ }^{1}$ the species and temperature gradients normal to the disk are equal everywhere on the disk. Thus, such a configuration has great potential for highly uniform chemical vapor deposition (CVD), ${ }^{2-5}$ and indeed commercial rotating-disk CVD reactors are now available.

In certain operating regimes, the equations describing the complex three-dimensional spiral fluid motion can be solved by a separation-of-variables transformation ${ }^{5,6}$ that reduces the equations to a system of ordinary differential equations. Sírictly speaking, the transformation is only valid for an unconfined infinite-radius disk anil buoyancy-free flow. Furthermore, only some boundary conditions are consistent with the transformation (e.g , temperature, gas-phase composition, and approach velocity all specified to be independent of radius at sc me distance above the disk). Fortunately, however, the transformed equations still provide a very good practical approximation to the flow in a finite-radius reactor over a large fraction of the disk (up to 90\% of the disk radius) when the reactor operating parameters are properly chosen, i.e., high rotation rates. ${ }^{1}$

In the limit of zero rotation rate, the rotating disk flow reduces to a stagnation-point flow, for which a similar separation-of-variables transformation is also available. Such flow configurations ("pedestal reactors") also find use in CVD reactors.

In this report we describe a model formulation and mathematical analysis of rotating-disk and stagnationpoint CVD reactors. Then we apply the analysis to a computer code called SPIN and describe its implementation and use.

An infinite-radius disk rotating below a fluid medium is a classic problem in fluid mechanics. ${ }^{6-8}$ Under these ideal conditions this problem has a solution that is an exact solution of the Navier-Stokes equations. Consequently, the heat and mass transfer near an infinite-radius rotating disk have been extensively studied. ${ }^{9,10}$ For CVD applications, Olander ${ }^{2,3}$ used a rotating disk to study deposition in the germaniumiodine system. Pollard and Newman ${ }^{5}$ performed a theoretical study of the deposition of $\mathrm{Si}_{\text {from }} \mathrm{SiCl}_{4}$ on a rotating-disk susceptor. They extended the von Karman similarity solution for isothermal flow ${ }^{6}$ by adding energy and species equations and incorporating temperature-dependent fluid properties to obtain an ordinary differertial equation boundary-value problem for the heat, mass, and momentum transfer. Hitchman et al. ${ }^{4}$ studied epitaxial $\mathrm{Si}$ deposition from $\mathrm{SiCl}_{4}$ in a iotating-disk reactor and analyzed their results in terms of the infinite-disk solution.

In this report we begin with the transformation (which is valid for either the rotating disk or stagnationpoint flow) derived by Evans and Greif ${ }^{11}$ and then extend the analysis to include the coupling of fluid flow with chemical kinetics. ${ }^{12}$ In addition to the fluid mechanics the model formulation includes the production and depletion of species due to reactions in the gas phase and at the surface, convective and diffusive transport of species in the gas phase, and transport to the deposition surface. The numerical model predicts deposition rates and gas-phase chemical species density profiles, surface coverages, gas velocities, and temperature profiles as a function of process parameters such as susceptor (i.e., the disk) temperature, disk-rotation rate, pressure, and flow rates.

The SPIN software depends on several other software packages. The chemical source terms in the governing equations are provided by the CHEMKIN ${ }^{13}$ package. The transport properties (viscosities, thermal 
conductivities, thermal diffusion coefficients, and diffusion coefficients) are evaluated with the TRANSPORT software package. ${ }^{14}$ The surface-reaction chemistry is modeled using the SURFACE CHEMKIN package. ${ }^{15}$ The numerical solution algorithm is implemented in the TWOPNT software. ${ }^{16}$ Familiarity with these packages is essential to preparing, reaction mechanisms and other input for use in SPIN. The supporting software packages have associated preprocessor programs, databases, and subroutine libraries. Thus, using the SPIN program requires the user to establish certain linkages between all the required modules. A general discussion on the philosophy of this highly modular approach and specific comments on how to establish the required linkages is found in Chapter IV of the SURFACE CHEMKIN Users Manual. ${ }^{15}$

The problem formulation and solution technique for the SPIN problem is highly analogous to the approach that we have taken to solve laminar premixed flame problems for combustion applications. The computational approach for these problems is documented in Grcar et al. ${ }^{17}$ and in Miller et al. ${ }^{18}$ The software implementation of the flame problem is documented in the users manual for PREMIX. ${ }^{19}$ Those familiar with PREMIX will see similarities in the SPIN software.

\section{ROTATING-DISK/STAGNATION-POINT FLOW EQUATIONS}

Consider a solid rotating surface of infinite extent in the $r, \theta$ plane (Figure 1) separated from a facing, parallel, porous non:otating surface by a distance $x=L$. A forced flow with purely axial velocity emerges from the porous surface and is directed toward the rotating one. The flow at $x=L$ approximates the inlet flow conditions in a cylindrical, rotating-disk, CVD reactor. The finite $x$ dornain in the present case results in a nonzero value of the radial pressure gradient.

Prior analyses vere limited in that there was no flexibility in specifying an inlet velocity. A recent analysis by Evans and Greif ${ }^{11}$ combined the stagnation and rotating disk flows for the situation of an infinite-radius, porous, nonrotating disk separated by a distance $L$ from an infinite-radius, nonporous, heated rotating disk. This combination of flows provides a good approximation to the flow field in a rotating disk reactor. The Evans and Greif analysis forms the foundation for the present model development. However, since predicting details of the chemical kinetics behavior is a main objective here, the present system also incorporates a species conservation equation for each species that occurs in the gas phase ${ }^{12}$. These equations account for convective and diffusive transport of species, as well as production and consumption of species by elementary chemical reastions. The conservation equations defining the boundary-value system that we solve are stated below.

Mixture continuity:

$$
\frac{1}{\rho} \frac{\partial \rho}{\partial t}=-\frac{\partial u}{\partial x}-2 V-\frac{u}{\rho} \frac{\partial \rho}{\partial x}=0
$$

Radial momentum:

$$
\rho \frac{\partial V}{\partial t}=\frac{\partial}{\partial x}\left(\mu \frac{\partial V}{\partial x}\right)-\rho u \frac{\partial V}{\partial x}-\rho\left(V^{2}-W^{2}\right)-\frac{1}{r} \frac{d p_{m}}{d r}=0
$$

Circumferential momentum:

$$
\rho \frac{\partial W}{\partial t}=\frac{\partial}{\partial x}\left(\mu \frac{\partial W}{\partial x}\right)-\rho u \frac{\partial W}{\partial x}-2 \rho V W=0
$$

Thermal energy:

$$
\rho c_{p} \frac{\partial T}{\partial t}=\frac{\partial}{\partial x}\left(\lambda \frac{\partial T}{\partial x}\right)-\rho c_{p} u \frac{\partial T}{\partial x}-\sum_{k=1}^{K_{g}}\left(c_{p_{k}} \rho Y_{k} V_{k} \frac{\partial T}{\partial x}+\dot{\omega}_{k} h_{k}\right)+S_{q}(x)=0
$$




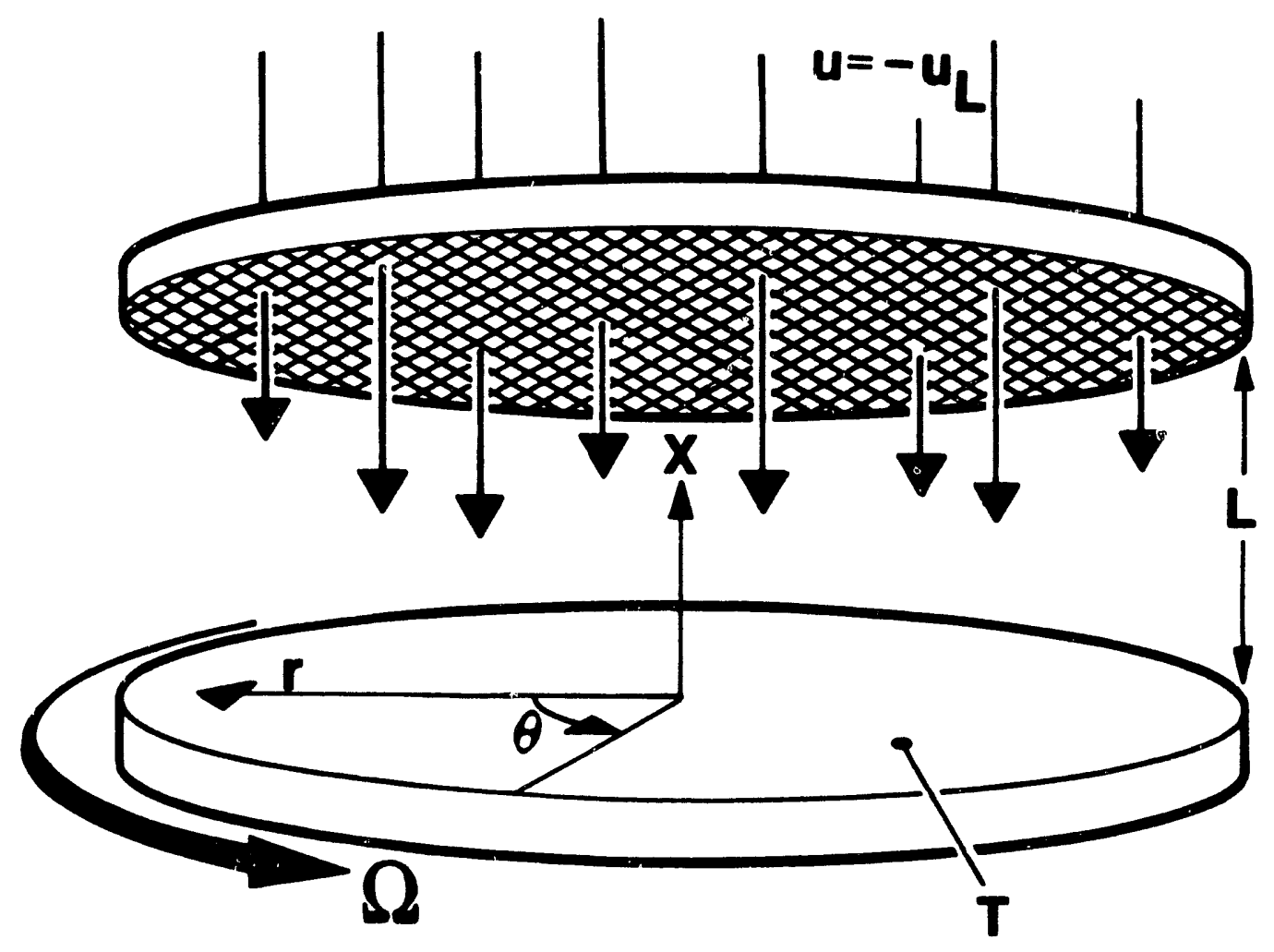

Figure 1. Sketch of the infinite-radius disk and inlet boundary conditions.

Species continuity:

$$
\rho \frac{\partial Y_{k}}{\partial t}=-\frac{\partial \rho Y_{k} V_{k}}{\partial x}-\rho u \frac{\partial Y_{k}}{\partial x}+M_{k} \dot{\omega}_{k}=0 \quad\left(k=1, \ldots, K_{g}\right)
$$

Equation of state:

$$
P=\frac{\rho R T}{\bar{M}}
$$

Sur 'ace-Species Conservation:

$$
\frac{d Z_{k}}{d t}=\frac{\dot{s}_{k}}{\Gamma_{n}}=0 \quad\left(k=1, \ldots, K_{s}\right)
$$

The objective of the SPIN software is to solve a steady-state problem, not a time-dependent one. Nevertheless, periodically the solution algorithm calls for computing a transient response. Therefore, the governing equations are stated in their transient form, even though we seek the solution when all time derivatives are exactly zero. The solution algorithm is discussed further in following sections.

In the governing equations the independent variables are $x$, the distance normal to the disk surface, and $t$, time. The dependent variables ar $\approx$ the velocities, the temperature $T$, the gas-phase species mass fractions $Y_{k}$, and the surface-species site fractions $Z_{k}$. The axial velocity is $u$, and the radial and circumferential velocities are scaled by the radius as $V=v / r$ and $W=w / r$, respectively. The mass density is given by $\rho$ and the specific heats by $c_{p}$. The molecular weight and specific enthalpy for species $k$ are given by $M_{k}$ and $h_{k}$. The viscosity and thermal conductivity are given by $\mu$ and $\lambda$. The universal gas constant is $R$. The ciremical production rate of species $\dot{\omega}_{k}$ by gas-phase reaction is presumed to result from a system of elementary chemical reactions that proceed according to the law of mass action. The chemical production 
rate of species by surface reaction is given by $\dot{s} . K_{g}$ is the number of gas-phase species and $K_{s}$ is the number $\mathrm{cf}$ surface species, not including bulk-phase species. The factor $\Gamma_{n}$ is the surface site density for site type $n$. The details of the chemical reaction rate formulation can be found in the user's manuals for the CHEMKIN ${ }^{13}$ and SURFACE CHEMKIN ${ }^{15}$ software. Details of the transport property (i.e., viscosities, thermal conductivities and diffusion coefficients) formulation can be found in the user's manual for the TRANSPORT ${ }^{14}$ software.

The term $(1 / r)\left(d p_{m} / d r\right) \equiv \Lambda$ in the radial momentum equation is taken to be constant and its value is computed as an eigenvalue of the problem. The pressure is assumed to be composed of two parts: an average thermodynamic pressure $P$ that is taken to be constant, and a spatially-varying component $p_{m}$ that appears in the radial momentum equation (2) ( c.f. Paolucci ${ }^{20}$ ).

The "surface-species conservation" equation states simply that in steady state the surface composition does not change. In some sense it could be considered a (possibly complex) boundary condition on the gas-pliase system. However, because the surface composition is determined as part of the solution, Eq. (7) should be considered part of the system of governing equations.

The SPIN code makes provision for dealing with the transport properties at the mixture-averaged (Fickian) level or at the full multicomponent level. At the mixture-averaged level, each species diffusion velocity is calculated in terms a diffusion coefficient and a species gradient,

$$
V_{k}=-\frac{1}{X_{k}} D_{k m} \frac{d X_{j}}{d x}-\frac{D_{k}^{T}}{\rho Y_{k}} \frac{1}{T} \frac{d T}{d x}
$$

where,

$$
D_{k m}=\frac{1-Y_{k}}{\sum_{j \neq k}^{K} X_{j} / \mathcal{D}_{j k}} .
$$

At the multicomponent level, the diffusion velocities are given as

$$
V_{k}=\frac{1}{X_{k} \bar{M}} \sum_{j=1}^{K_{g}} M_{j} D_{k j} \frac{d X_{j}}{d x}-\frac{D_{k}^{T}}{\rho Y_{k}} \frac{1}{T} \frac{d T}{d x} .
$$

Both formulations have an ordinary diffusion component and may have a thermal diffusion (Soret effect) component. In these expressions, $X_{k}$ is the mole fraction for the $k$ th species, $\mathcal{D}_{j k}$ is the binary diffusion coefficient matrix, $D_{k j}$ is the matrix of ordinary multicomponent diffusion coeficients, and $D_{k}^{T}$ is the thermal diffusion coefficient for the $k$ th species. Thermal diffusion often plays an important role in CVD problems. In the presence of strong temperature gradients, thermal diffusion causes high molecular-weight species in a low molecular-weight carrier to diffuse rapidly toward the low-temperature region. ${ }^{21}$ The multicomponent and mixture transport propertics are evaluated from the pure species properties using the averaging procedures given by Dixon-Lewis ${ }^{22}$ computed with the TRANSPORT package of Fortran subroutines. ${ }^{14}$

Mass conservation requires that $\sum_{k=1}^{K_{g}} Y_{k} V_{k}=0$. However, a consequence of using the Fickian mixtureaveraged diffusion coefficient defined in Eq. (9) to define a diffusion velocity in Eq. (8) is that mass is not always conserved, i.e., $\sum_{k=1}^{K_{o}} Y_{k} V_{k} \neq 0$. Therefore, at this level of closure of the transport formulation some corrective measures must be taken. The user of SPIN has several options. One is for the code to apply an ad hoc correction velocity, defined as

$$
V_{c}=-\sum_{k=1}^{K} Y_{k} V_{k}
$$

When this correction velocity (independent of species $k$ ) is added to all the species diffusion velocities as computed from Eq. (8), mass conservation is assured.

Another option in SPIN is to account for the deficiencies of the mixture-averaged closure of the multicomponent transport problem and to assure mass conservation is to solve only $K_{g}-1$ gas-phase species 
conservation equations and determine the remaining mass fraction by requiring $\sum_{k=1}^{K_{g}} Y_{k}=1$. (The mixtureaveraged transport closure is asymptotically correct in the trace-species limit.) In cases where one species is present in large excess (such as a carrier gas in a CVD reactor), this is a reasonable option. The carrier gas composition is conveniently determined as

$$
Y_{K_{0}}=1-\sum_{k=1}^{K_{0}-1} Y_{k}
$$

The default for this option is to consider the last-named gas-phase species in the CHEMKIN input $\left(K_{g}\right)$ as the species for which a conservation equation is not solved. SPIN also provides for dynamically determining the largest specie; concentration at cach mesh point and removing its conservation equation from the system of equations. Invoking any of these options is accomplished by using combinations of the keywords described in Chapter V.

The source term in the thermal energy equation $S_{q}(x)$ is a spatially distributed thermal energy source that we assume is in the form of a Gaussian:

$$
S_{q}(x)=\dot{q} \frac{1}{w_{s}} \sqrt{\frac{3}{\pi}} \exp \left[\frac{-3\left(x-x_{s}\right)^{2}}{w_{s}^{2}}\right]
$$

To use this expression, the user is expected to specify three parameters: $\dot{q}, w_{s}$, and $x_{s}$. The parameter $\dot{q}$ is the total energy integrated over its full spatial extent. Implicit in Eq. (12) is the fact that

$$
\dot{q}=\int_{-\infty}^{\infty} S_{q}(x) d x
$$

The distribution is centered at $x=x_{s}$ and $w_{s}$ is the $2 \sigma$ half-width of the distribution. (The integral of $S_{q}(x)$ from $-w_{s}$ to $w_{s}$ includes $95 \%$ of the total added energy $\dot{q}$.)

The disk boundary condition becomes relatively complex in the presence of heterogeneous surface reactions. The gas-phase mass flux of each species to the surface $j_{k}$ is balanced by the creation or depletion rate of that species by surface reactions, i.e.,

$$
j_{k}=\dot{s}_{k} \quad\left(k=1, \ldots, K_{g}\right) .
$$

The gas-phase mass flux of species $k$ at the surface is a combination of diffusive and convective processes,

$$
j_{k}=\rho Y_{k} u+\rho Y_{k} V_{k} \quad\left(k=1, \ldots, K_{g}\right),
$$

where $u$ is the bulk normal fluid velocity at the surface and $V_{k}$ is the diffusion velocity of the $k$ th species. The bulk normal fluid velocity at the surface is computed from the surface reaction rates summed over all the gas-phase species $K_{g}$,

$$
u=\sum_{k=1}^{K_{\rho}} \frac{\dot{s}_{k} M_{k}}{\rho} .
$$

Even though the susceptor surface is solid, there is a bulk fluid velocity into the surface (the Stefan velocity) that accounts for the mass of solids deposited. This bulk velocity at the surface is usually small, and thus the boundary movement due to the deposition is neglected. That is, the problem is solved on in a fixed spatial domain. While SPIN predicts the surface growth rate, it does not adjust the computational domain to account for small changes resulting from surface growth.

SPIN provides two options for treating the thermal-energy boundary condition on the deposition surface. The first is to simply specify the surface temperature. If the temperature is controlled or measured directly, this option is usually the one of choice. However, some problems require that the surface temperature be 
predicted as a part of the solution. The appropriate boundary condition is derived from a surface energy balance. Exothermicity (or endothermicity) of surface reactions contribute to the energy balance at an interface. Diffusive and convective fluxes in the gas phase are balanced by therma! radiative and chemical heat release at the surface. This balance is stated as

$$
\lambda \frac{\partial T}{\partial x}-\sum_{k=1}^{K_{g}} \rho Y_{k}\left(V_{k}+u\right) h_{k}=\sigma \epsilon\left(T^{4}-T_{w}^{4}\right)+\sum_{k=K_{!}^{!}}^{K_{b}^{1}} \dot{s}_{k} M_{k} h_{k}+\dot{P} .
$$

In the radiation term, $\sigma$ is the Stefan-Boltzmann constant, $\epsilon$ is the surface emissivity, and $T_{w}$ is the temperature to which the surface radiates. The summation on the right-hand side runs over all surface and bulk species. $K_{b}^{f}$ and $K_{b}^{l}$ are the SURFACE CHEMKIN notations for the indices that identify the first surface species and the last bulk species. By substituting Eqs. (14) and (15) into Eq. (17), the energy balance can be written in a more compact form as

$$
\lambda \frac{\partial T}{\partial x}=\sigma \epsilon\left(T^{4}-T_{w}^{4}\right)+\sum_{k=1}^{K} \dot{s}_{k} M_{k} h_{k}+\dot{P} .
$$

The reaction-rate summation on the right-hand side runs over all species, including the gas-phase species. The term $\dot{P}$ represents an energy source in the surface itself, such as might bo generated by resistance heating.

The SURFACE CHEMKIN package requires as input the mass densities $\rho_{k}$ of the bulk species. The SPIN code uses these densities to convert the rate of production of a bulk species (in moles $/ \mathrm{cm}^{2} / \mathrm{sec}$ ) into a thickness growth rate $G$ (in $\mathrm{cm} / \mathrm{sec}$ ). The needed relationship is

$$
G=\sum_{k=K_{b}^{\prime}}^{K_{b}^{\prime}} \frac{\dot{s}_{k} M_{k}}{\rho_{k}}
$$

When solving for the flow induced by the rotation of a disk in an infinite, otnerwise quiescent fluid, the axial velocity $u$ at $x=L$ is part of the solution. However, for the case corresponding to injection of the gas through a nonrotating porous surface, $u$ is the specified inlet velocity at neight $x=L$. This gives us the flexibility of either "forcing" or "starving" the inlet flow compared to the natural flow induced by the spinning disk itself. It is always necessary to specify the inlet velocity in the case of a stagnation-point flow.

The other boundary conditions on the fluid flow fields are relatively simple. The temperature and species composition at $x=L$ (the reactor inlet) are specified. Normally, the radial and circumferential velocities are zero at $x=L$. However, SPIN makes the provision for a linearly varying radial velocity or a specified spin rate at $x=L$. In these cases, $V=v / r=a_{i}$ or $W=w / r=o_{i}$, where $a_{i}$ and $o_{i}$ are specified parameters. The radial velocity on the disk is zero, the circumferential velocity is determined from the spinning rate $W=\Omega$. 


\section{NUMERICAL SOLUTION METHOD}

The TWOPNT software provides the implementation of the hybrid Newton/time-step algorithm that we use to solve the governing equations in SPIN. In this section we only summarize the salient features of the algorithm and refer to the TWOPNT manual ${ }^{16}$ for more detail.

The numerical solution procedure begins by m.aking finite difference approximations to reduce the ordinary differential equation boundary value problem to a system of algebraic equations. The initial approximations are usually on a coarse mesh that may have as few as five or six points. After obtaining a solution on the coarse mesh, new mesh points are added in regions where the solution or its gradients change rapidly. An initial guess for the solution on the finer mesh is obtained by interpolating the coarse mesh solution. This procedure continues until no new mesh points are needed to resolve the solution to the degree specified by the user. This continuation from coarse to fine meshes significantly enhances the efficiency of the algorithm. A solution to the systern of algebraic equations is first attempted by the damped modified Newton algorithm. However, if the Newton algorithm fails to converge, then the solution estimate is conditioned by a time integration. This provides a new starting point for the Newton algorithm that is closer to the solution, and thus more likely to be in the domain of convergence for Newton's method. As the mesh becomes finer, the estimate interpolated from the previous mesh is likely to be within the domain of convergence of Newton's method.

There are many controls that can be used to influence the course of the Newton search and the time evolution. Most have default values that need not be changed for most problems. However, for difficult problems, the user may spend considerable effort working with the keywords in the "Method Options" section of Chapter V to help TW PNT cope with convergence difficulties.

\section{Finite Difference Approximations}

The first tisk in solving the deposition problem is to discretize the governing conservation equations. We use finite difference approximations on a nonuniform grid with points numbered by $j$ from 1 at the disk boundary to $J$ at the inlet boundary.

The "convective" terms, such as $p u \partial V / \partial x$ in Eq. (2) are approximated by first-order upwind formulas, as

$$
\rho u \frac{\partial V}{\partial x} \approx \rho_{j} u_{j}\left(\frac{V_{j+1}-V_{j}}{x_{j+1}-x_{j}}\right) .
$$

The $u$ velocity is always negative in these problems (flowing from the inlet at large values of $x$ toward the substrate at $x=0$ ), so the upwind differencing calls for the derivative to be formed between $j+1$ and $j$. Upwind differences cause substantial artificial diffusion, especially on coarse mesh networks. However, we have found that they lead to much more reliable convergence on coarse meshes early in the iteration procedure. Ultimately, because the meshes are adaptively refined, the artificial diffusion is very small in the final solution.

Th. f first derivative in the summation term in the energy equation (4) is always approximated by a central difference formula,

$$
\left(\frac{\partial T}{\partial x}\right)_{j} \approx\left(\frac{h_{j-1}}{h_{j}\left(h_{j}+h_{j-1}\right)} T_{j+1}+\frac{h_{j}-h_{j-1}}{h_{j} h_{j-1}} T_{j}-\frac{h_{j}}{h_{j-1}\left(h_{j}+h_{j-1}\right)} T_{j-1}\right),
$$

where the mesh intervals $h_{j}=x_{j+1}-x_{j}$. The coefficients in the summation are evaluated at $j$. 
The second-derivative diffusion terms, such as that in the energy equation, are appioximated by the following second order central difference:

$$
\frac{\partial}{\partial x}\left(\lambda \frac{\partial T}{\partial x}\right)_{j} \approx\left(\frac{2}{x_{j+1}-x_{j-1}}\right)\left[\lambda\left(\frac{T_{j+1}-T_{j}}{x_{j+1}-x_{j}}\right)-\lambda\left(\frac{T_{j}-T_{j-1}}{x_{j}-x_{j-1}}\right)\right]
$$

The coefficient $\lambda$ in this formula (at $j \pm 1 / 2$ ) are evaluated using the averages of the dependent variables between mesh points.

The diffusive terms in the species conservation equations are analogous to the diffusive term in the energy equation. However, because we express them in terms of diffusion velocities, they at first appear different. In Eq. (5), the diffusive term is approximated as

$$
\frac{\partial \rho Y_{k} V_{k}}{\partial x} \approx \frac{\rho_{j+1 / 2} Y_{k, j+1 / 2} V_{k, j+1 / 2}-\rho_{j-1 / 2} Y_{k, j-1 / 2} V_{k, j-1 / 2}}{x_{j+1 / 2}-x_{j-1 / 2}}
$$

The ordinary multicomponent diffusion and thermal diffusion fluxes (Eq. 10) are approximated at the $j \pm 1 / 2$ positions as illustrated by

$$
\begin{aligned}
\left(\rho Y_{k} V_{k}\right)_{j+1 / 2}= & \left(\frac{\rho M_{k}}{\bar{M}^{2}}\right)_{j+1 / 2} \sum_{l=1}^{K_{g}}\left(M_{l} D_{k, l}\right)_{j+1 / 2}\left(\frac{X_{l, j+1}-X_{l, j}}{x_{j+1}-x_{j}}\right) \\
& +\left(\frac{D_{k}^{T}}{\rho Y_{k} T}\right)_{j+1 / 2}\left(\frac{T_{j\lrcorner .1}-T_{j}}{x_{j+1}-x_{j}}\right) \cdot(24)
\end{aligned}
$$

An analogous finite-difference expression is used for the mixture-averaged approximation to the diffusion fluxes. Since the mole fraction of a species can be zero, wi: avoid difficulties by forming $\rho Y_{k} V_{k}$, which is the expression needed in Eq. (4), rather than $V_{k}$ itself $\left(Y_{k}=\chi_{k} M_{k} / \bar{M}\right)$.

All the nondifferentiated terms, such as the chemical production rate terms, are evaluated at the mesh points $j$. Coefficients not appearing within derivatives are also evaluated at the mesh points.

\section{Starting Estimates}

SPIN needs a starting estimate of the solution from which to begin its iteration. The general form of this estimate is shown in Fig. 2. The estimate assumes there is a reaction zone in which the inlet gas composition changes to the product composition. The user estimates the location and thickness of this reaction zone and the composition of the products. Within the reaction zone the program uses linear interpolation between the initial and final values for the reactants and products. On the inlet side of the reaction zone the reactant species profiles are flat at the inlet values. On the disk side, the product species are flat at the estimated product values. Note that a species can be both a reactant and a product. For example, the helium carrier and any excess silane could be both reactants and products in a silicon deposition system. Species can also be identified as "intermediates." Intermediates, such as short-lived radical species, are assumed to have a Gaussian profile that peaks in the center of the reaction zone. The peak height is specified in the input to the program, and the Gaussian's width is such that the profile is at $1 / 10$ of its peak value at the edges of the reaction zone.

We find that the shape of the assumed initial species profiles is not too important. Smoother shapes have no apparent effect on the convergence properties of the method. Moreover, since the starting profiles are typically evaluated on a very coarse mesh, it makes little difference whether the underlying functions are smooth or not. 


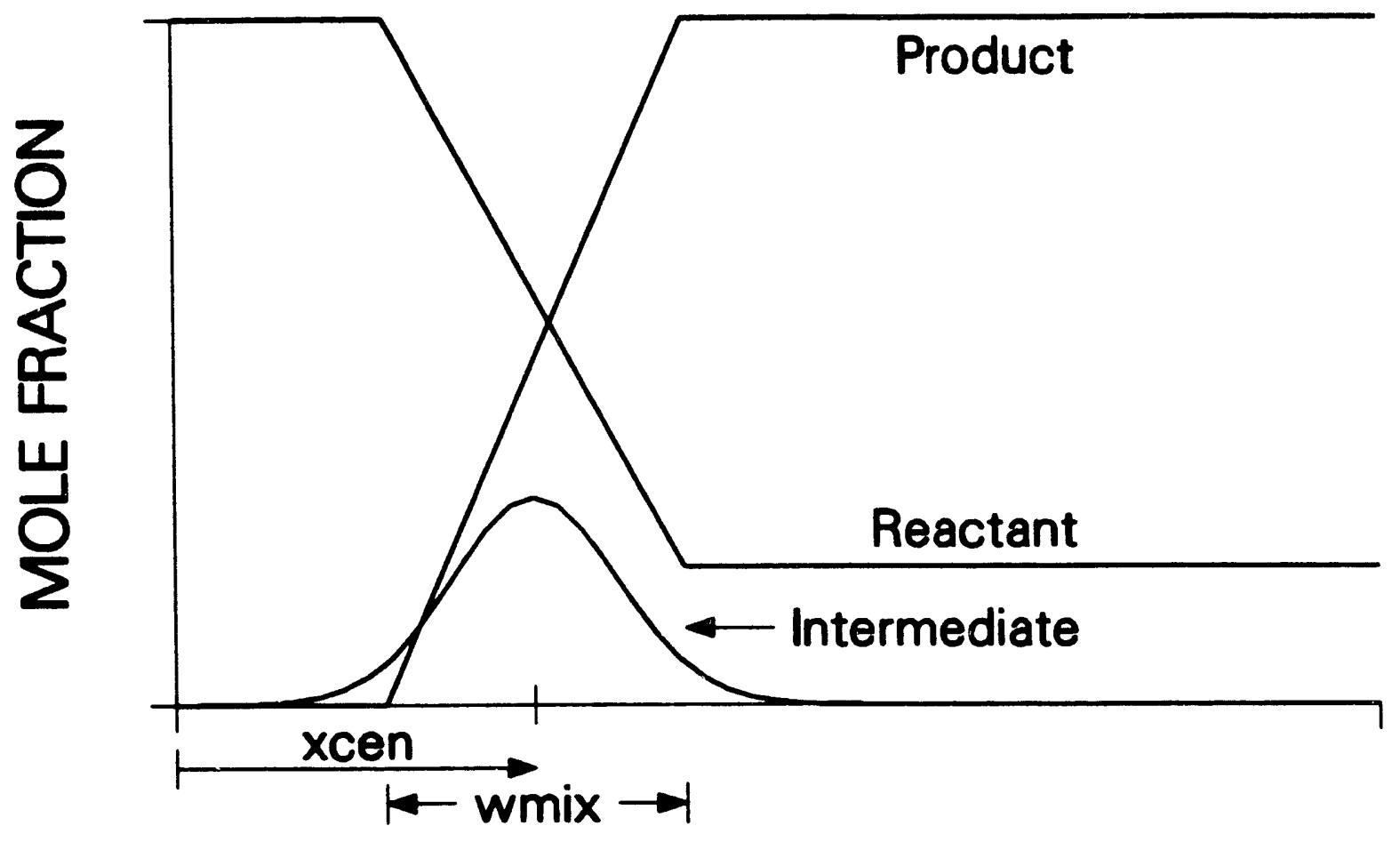

Figure 2. The general form of the starting estimate.

\section{Continuation Start-up Procedure and User-Specified Temperature Profile}

It is sometimes preferred to model the deposition using a known temperature profile. For example, temperature data may be available experimentally and using them may facilitate interpreting chemistry data from the reactor. If a specified temperature profile is used the energy equation is not solved. There are computational advantages to using a known temperature profile. The most severe nonlinearities in chemical kinetics come from the exponential dependence on temperature of the reaction rates. Thus, eliminating the temperature from the iteration makes the problem considerably easier to solve.

We find that even if the energy equaticn is to be solved for the temperature distribution, the iteration often converges more reliably if the species profiles are first computed using a fixed temperature profile. The SPIN program therefore provides for the following three-stage procedure to solve the fully coupled problem. However, either or both the first two stages can be skipped by using appropriate Keywords.

1. The program solves for just the velocity fields (and temperature, if not specified as fixed using the TEMP and TGIV Keywords), neglecting all chemistry and species equations. For chemical vapor deposition problems where there is a large nonreacting carrier gas concentration, the nonreacting problem provides a good approximation of the temperature and velocity fields. This stage may be bypassed by using the NONR Keyword.

2. The full set of species and flow equations is solved, using the temperature field from the first step and ignoring the energy equation. Especially for problems with large carrier-gas concentrations, the temperature profile from step one is usually quite close to the final profile, and omitting the energy equation at this point eases solution by eliminating a strong nonlinearity (due to the Arrhenius form of the rate 
expressions). If the TGIV Keyword is used, the temperature profile used at this stage is the one specified through the TEMP Keywords. This stage can be bypassed by using the NOFT Keyword.

3. The energy equation is included and the fully coupled problem is solved, using the output from stage two as the initial guess. If the temperature profile was specified using the TGIV, TEMP combination, this stage is skipped because stage 2 completes the calculation.

This multi-stage continuation procedure is usually more efficient than attempting to solve the fully coupled problem directly.

The user input for the temperature profiles is in the form of position-temperature pairs. In general, the mesh used by the SPIN program will not be the same as the one on which the given temperature estimate is defined. The program uses a linear interpolation of the given temperatures onto the current mesh. Thus, especially in the case where the energy equation is not included, it is important for the user to provide enough temperature points that the profile is well-resolved and relatively smooth.

\section{Modified Damped Newton's Method}

The TWOPNT software implements the computational algorithms, which are documented completely in the TWOPNT user's manual. In this and following sections, the solution method is introduced brieflymainly to establish some nomenclature for the keywords and the sensitivity analysis.

After discretization on a given mesh, we have a system of nonlinear algebraic equations that we attempt to solve by a damped Newton's method. Newton's method determines a sequerice of iterations or approximate solutions that approach the true solution. For the sake of notational ease we call these approximate solution vectors $\phi$. When any arbitrary $\phi$ is substituted into the finite difference analog of Eqs. (1-7), they do not equal zero as they would if the true solution were substituted; in general they equal a residual vector that we will call $F$. Thus our purpose is to find a vector $\phi$ that satisfies

$$
F(\phi)=0 .
$$

In our case the vector $\phi$ is composed as follows:

$$
\phi=\left(z, u_{1}, V_{1}, W_{1}, T_{1}, \Lambda_{1}, Y_{1,1}, \ldots, u_{J}, V_{J}, W_{J}, \Lambda_{J}, T_{J}, Y_{1, J}\right)^{T} .
$$

The corresponding $F$ vector is composed of the residuals of the surface species equations, energy equation, the velocity equations, the gas-phase species equations, and the pressure eigenvalue equation. The ordering of the $F$ vector corresponds to the order of the $\phi$ vector; it begins with the residuals of the surface species, then the residuals at the first node (the disk), followed by the residuals at the interior mesh points, and finally the residuals at the inlet boundary, $j=J$.

If the initial estimate $\phi^{(0)}$ of the solution is sufficiently good, then Newton's method produces a sequence $\left\{\phi^{(n)}\right\}$ that converges to the solution of the nonlinear equations $F(\phi)$. The purest form of the algorithm,

$$
\phi^{(n+1)}=\phi^{(n)}-\left(\frac{\partial F}{\partial \phi}\right)_{\phi(n)}^{-1} F\left(\phi^{(n)}\right)
$$

is too expensive and delicate to be used in practice. On the one hand, evaluation of the Jacobian matrices $\partial F / \partial \phi$ by numerical or other means is time consuming, and on the other hand, convergence usually requires a very good initial estimate $\phi^{(0)}$. TWOPNT employs the usual remedies. First, the Jacobian matrix is replaced by one, $J^{(n)}$, inherited from a previous step of the algorithm. Second, the full step from $\phi^{(n)}$ to $\phi^{(n+1)}$ may be cut short by a damping parameter $\lambda^{(n)}$. In this way the iteration becomes

$$
\phi^{(n+1)}=\phi^{(n)}-\lambda^{(n)}\left(J^{(n)}\right)^{-1} F\left(\phi^{(n)}\right)
$$


where, $0<\lambda^{(n)} \leq 1$, and

$$
J^{(n)}=J^{(n-1)} \text { or } J^{(n)}=\left(\frac{\partial F}{\partial \phi}\right)_{\phi(n)}
$$

Of course, the inverse Jacobian matrix in Eq. (27) is not computed; instead a system of linear equations $J^{(n)} \Delta \phi^{(n)}=F\left(\phi^{(n)}\right)$ is solved for the undamped correction vector $\Delta \phi^{(n)}$ using the LINPACK software. ${ }^{23}$ The TWOPNT documentation provides a full description of the damping strategy.

\section{Jacobian Matrix}

TWOPNT requires that the application code (SPIN) form Jacobian matrices and factor them into upper and lower triangular parts. The Jacobian is a large banded (actually block tridiagonal) matrix. In principle, we could derive and evaluate analytic expressions for the Jacobian elements. However, because this is a difficult and error-prone task, and because the modified Newton method can work well with old (and hence inaccurate) Jacobians, the effort to develop analytic Jacobian expressions is not warranted. Instead, we form the elements of the Jacobian by finite difference perturbations in the manner suggested by Curtis et al. ${ }^{24}$ Specifically, we evaluate a one-sided finite difference formula,

$$
J_{i, j} \approx \frac{F_{i}\left(\phi_{j}+\delta\right)-F_{i}\left(\phi_{j}\right)}{\delta}
$$

where

$$
\delta=r \times \phi_{j}+a .
$$

We choose the relative and absolute perturbations, $r$ and $a$, to be the square root of the computer's unit roundoff. These parameters, therefore, may depend on the user's particular computer. The Jacobian factorization is accomplished with the LINPACK software.

\section{Adaptation}

We have found that starting the solution search on a coarse mesh has several advantages. One is that the Newton iteration is more likely to converge from a bad guess on a coarse mesh than on a fine mesh. Since the iteration begins from a user-specified "guess" at the solution, it is likely that many iterations will be required. But since the number of variables is small on a coarse mesh, the cost per iteration is relatively small. Ultimately, of course, the solution must be obtained on a fine mesh to be accurate. However, as the solution is computed on each successively finer mesh, the starting estimates are better and, in general, the solution on one mesh lies within the domain of convergence of Newton's method on the next finer mesh. ${ }^{25}$ Thus, even though the cost per iteration is increasing on refined meshes, the number of required iterations is decreasing.

The adaptive placement of the mesh points to form the finer meshes is done in such a way that the total number of mesh points needed to represent the solution accurately is minimized. Specifically, we place the mesh points in the following way. We exclude variables from consideration in the adaptation that are below a certain floor value. This avoids adapting on variables that are essentially zero, but due to rounding errors may show locally high derivatives. To resolve the gradients we bound the variation in the solution components between mesh points as

$$
\left|\phi_{n, j}-\phi_{n, j-1}\right| \leq \operatorname{GRAD} \times\left(\max \phi_{n}-\min \phi_{n}\right),
$$


and to resolve the curvature in the solution we bound the variation in the solution's derivatives between mesh points by

$$
\left|\left(\frac{d \phi_{n}}{d x}\right)_{j}-\left(\frac{d \phi_{n}}{d x}\right)_{j-1}\right| \leq \operatorname{CURV} \times\left(\max \frac{d \phi_{n}}{d x}-\min \frac{d \phi_{n}}{d x}\right) .
$$

In the program, we evaluate the above expressions between each of the mesh points. In each of the subintervals where the inequality is not satisfied, a new mesh point is placed at the midpuint of the subinterval. The parameters GRAD and CURV are specified by the user through Keyword parameters (Chapter V). The starting estimate for the dependent variable vector $\phi$ is determined by a linear interpolation of the coarse mesh solution onto the new finer mesh. After determining a converged solution on this new fine mesh, the adaptation procedure is performed once again. A sequence of solutions on successively finer meshes is computed until the inequalities in Eqs. (32) and (33) are satisfied between all mesh points.

\section{Time Stepping}

Even though the transient path is not of interest here, the steady state deposition solution that we seek is the result of a physically transient process. Determining the steady solution by solving the transient equations is a very reliable, but often slow, process. Therefore, we attempt to use a Newton method that converges very rapidly, when it converges. When it does not, then we condition the guess by evolving it in time.

SPIN (through TWOPNT) solves the transient parabolic system of partial differential equations with a first-order backward Euler method. In this method, the time derivatives are approximated by finitc differences as illustrated by

$$
\rho c_{p} \frac{\partial T}{\partial t} \approx \rho_{j}^{n+1} c_{p_{j}} \frac{T_{j}^{n+1}-7_{j}^{n}}{\Delta t},
$$

where the superscript $n$ indicates the time level and $\Delta t$ represents the size of the time step. All other terms are approximated with finite differences as before, but at time level $n+1$. Since all variables are known at time level $n$, the discretized problem is just a system of nonlinear algebraic equations for the dependent variable vector $\phi$ at time level $n+1$.

To sclve the system for each time step we use the same Newton method that we attempted to use to solve the steady state boundary value problem in the first place. However, the transient problem is much more likely to converge. It should always converge for a sufficiently small time step. From a physical point of view, as the time step approaches zero, the solution for $t^{n+1}$ should approach the initial condition or the the solution at $t^{n}$; thus convergence should be rapid and reliable. From a mathematical point of view, the Jacobian of the transient system has a factor $1 / \Delta t$ on the diagonal. Therefore, the condition number of the Jacobian will be reduced as the time step is reduced. The objective is to choose a time step that is large enough to make progress toward the steady solution, but not so large that the transient method also has convergence difficulties. We typically use time steps of around one to ten microseconds, although there are often cases where much smaller time steps are needed.

Through communication via keywords, SPIN's user can control the course of the transient solution. The number of time steps and their size can be specified, and the time-step size can be increased by a certain factor after a certain number of steps. If convergence difficulties are encountered in attempting to solve any time step, SPIN will reduce the size of the time step by a factor specified by the user. All these control keywords are described in Chapter V. 


\section{Sensitivity Analysis}

Sensitivity analysis is a way to understand quantitatively how the solution to a model depends on parameters in the model. Once the Jacobian of the residual function has been computed for the purposes of solving the boundary value problem, then the sensitivity coefficients can be computed easily. These techniques have been developed and used in the chemical engineering literature for some time. ${ }^{26-28}$ We find that sensitivity analysis is often an invaluable tool in helping to interpret the results of a deposition model. Coffee and Heimerl ${ }^{29,30}$ have also discussed the use of sensitivity analysis in flame modeling, although their computational method is quite different from the one we use here. Also, Rueven et al., ${ }^{31}$ using model problems, have explored various ways that sensitivity analysis can be applied to flames.

Here we consider the first order sensitivity coefficients of the solution profiles with respect to the reaction rate coefficients. To specify the sensitivity coefficients, let us begin with the boundary value problem stated in the abstract notation thai we introduced in Eq. (25),

$$
F(\phi ; \alpha)=0 .
$$

Note, however, that now we have introduced the idea that the equation may be parametrized by some parameters $\alpha$. In our case, these $\alpha$ 's will be the "A-factors" of the reaction rate coefficients. By differentiating Eq. (35) with respect to $\alpha$ we obtain a matrix equation for the sensitivity coefficients.

$$
\frac{\partial F}{\partial \phi} \frac{\partial \phi}{\partial \alpha}+\frac{\partial F}{\partial \alpha}=0
$$

The matrix $\partial F / \partial \phi$ is the Jacobian of the original system and $\partial F / \partial \alpha$ is the matrix of partial derivatives of $F$ with respect to the parameters. We will think of the $\partial F / \partial \alpha$ matrix column by column, with each column irdicating the dependence of the residual vector $F$ on a parameter. There are as many columns as there are parameters, i.e., reactions. The sensitivity coefficients are defined by $\partial \phi / \partial \alpha$. This matrix contains quantitative information on how each reaction rate coefficient affects the temperature and species profiles at each point in the reactor. The sensitivity matrix has a structure similar to that of the $\partial F / \partial \alpha$ matrix. That is, each column shows the dependence of the solution vector on a particular reaction rate coefficient.

The Jacobian and its LU factorization are already available from the solution of the original system by Newton's method, and the parameter derivatives are computed by finite differences in a manner similar to computation of the Jacobian. Therefore, the linear system of equations (36) is readily solved for each column of the sensitivity matrix, corresponding to the sensitivities of the solution vector to each of the $I$ reaction rates (gas plus surface reactions). The LINPACK software ${ }^{23}$ is used to perform these computations. It is set up so that the Jacobian is factored only once, and each column of the sensitivity matrix is computed by back substitution, a relatively inexpensive operation.

We find that some manipulation of the raw sensitivity coefficients makes them more useful. We compute normalized sensitivity coefficients in the form of logarithmic derivatives, i.e.,

$$
\frac{\alpha_{i}}{Y_{k}} \frac{\partial Y_{k}}{\partial \alpha_{i}}
$$

or

$$
\frac{\alpha_{i}}{T} \frac{\partial T}{\partial \alpha_{i}}
$$

In interpreting chemical information, we are more often interested in molar quantities, rather than mass quantities, so we compute the sensitivity coefficients in terms of mole fractions, i.e.,

$$
\frac{\alpha_{i}}{X_{k}} \frac{\partial X_{k}}{\partial \alpha_{i}}=\frac{\alpha_{i}}{Y_{k}} \frac{\partial Y_{k}}{\partial \alpha_{i}}-\alpha_{i} \bar{M} \sum_{j=1}^{K} \frac{1}{M_{j}} \frac{\partial Y_{k}}{\partial \alpha_{i}}
$$


where $X_{k}$ are the mole fractions and $\bar{M}$ is the mean molecular weight.

It is sometimes easier to interpret sensitivity coefficients that are normalized by the maximum value of a dependent variable rather than the local value. For example, we might modify Eq. (37) as

$$
\frac{\alpha_{i}}{Y_{k}^{\max }} \frac{\partial Y_{k}}{\partial \alpha_{i}},
$$

where $Y_{k}^{\max }$ is the maximum va' $\lambda$ e of the $k$ th species anywhere on the mesh. This normalization avoids artificially high sensitivity coefficients in regions where the mass fractions are approaching zero, and thus subject to numerical errors. SPIN reports the normalized sensitivity coefficients as represented by Eq. (38) and (39). Any further normalization can be done by the user in their own post-processing software.

SPIN considers as parameters $\alpha$ the forward rate constant "A-factors" for both the gas-phase and surface reaction mechanisms. Sensitivity coefficients are computed for all dependent variables at all mesh points (including the surface composition). Thus one can determine the effect of all reactions on all components of the solution.

\section{PROGRAM STRUCTURE}

In addition to input directly from the user, the SPIN program depends on data and subroutines from the CHEMKIN ${ }^{13}$ SURFACE CHEMKIN, ${ }^{15}$ and TRANSPORT packages. ${ }^{14}$ (Complete information on these software packages is available in their respective user's manuals.) Therefore, to solve a deposition problem the user should write a command procedure to orchestrate this process. The command procedure automatically executes several programs, accesses several databases, loads subroutines from several libraries, and passes files from one process to another. Figure 3 shows the relationships between these various components.

The first step is to execute the CHEMKIN Interpreter. The CHEMKIN Interpreter reads (Unit 15) usersupplied information about the species and chemical reactions for a particular gas-phase reaction mechanism. It then extracts further information about the species' thermodynamic properties from a database (Unit 17). This information is stored on the CHEMKIN Linking File (Unit 25), a file that is needed by the transport property fitting program (TRANFIT) the SURFACE CHEMKIN Interpreter, and later by the CHEMKIN subroutine library, which will be accessed by the SPIN program. The printed output from the CHEMKIN Interpreter is written to Fortran Unit 16.

The next program to be executed is the transport property fitting program, TRANFIT. It needs input from a transport property database (Unit 31) and from the CHEMKIN subroutine library. Its purpose is to compute polynomial representations of the temperature-dependent parts of the individual species viscosities, thermal conductivities, and the binary diffusion coefficients. Like the CHEMKIN Interpreter, the TRANFIT program produces a Linking File (Unit 35) that is later needed by the transport property subroutine library, which will evaluate mixture properties during the course of the deposition computation. Printed output from TRANFIT is written to Unit 6.

The SURFACE CHEMKIN Interpreter must also be executed after the CHEMKIN Interpreter has been run, because it relies on gas-phase species and element information in the CHEMKIN Linking file. The SURFACE CHEMKIN Interpreter reads (from Unit 15) user-supplied information about surface and bulk species names, site types, and surface reactions, and optional thermochemical information (Unit 17). This information is combined into a SURFACE CHEMKIN linking file (Unit 26), which will be used by the SURFACE CHEMKIN subroutine library and by the SPIN program. The printed output from the SURFACE CHEMKIN Interpreter is written to Unit 16. 


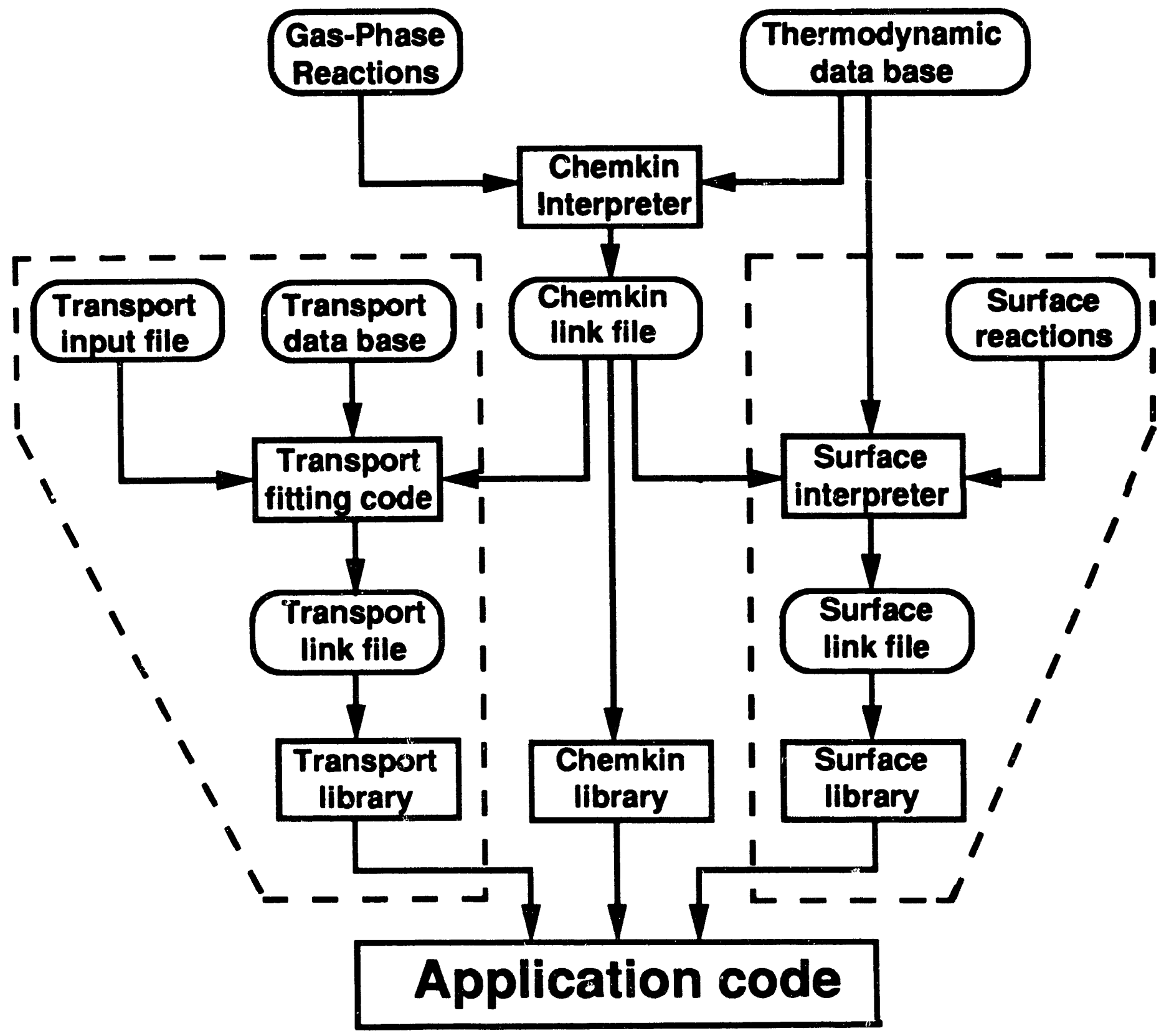

Figure 3. Relationship of the SPIN program to the CHEMKIN, SURFACE CHEMKIN, and TRANSPORT preprocessors and the associated input and output files. 
The SPIN program makes appropriate initialization calls to the CHEMKIN, SURFACE CHEMKIN, and TRANSPORT subroutine libraries. The purpose of the initialization is to read the Linking Files and set up the internal working and storage space required by all subroutines in the libraries.

The input that defines a particular deposition system and the parameters needed to solve it are read by the SPIN program in a Keyword format from Unit 5. In addition, there is a provision for the SPIN program to begin its iteration from a previously computed solution. In this case the old solution is read from a restart file assigned to Unit 14. The SPIN program produces printed output on Unit 6 and it saves the solution on Unit 15. The saved file can be used either to restart the same problem and continue iterating, or it can be read as a starting estimate for a different deposition which, for example, might have different reactant species compositions or flow rates.

\section{User-Supplied Program}

The SPIN program is written as a subroutine for which the user must write a small main program that opens all appropriate files, allocates the working storage, and calls the SPIN program through its subroutine interface. Figure 4 shows an example of the main, "driver" program.

The logical, integer, real, and character working space is allocated by the parameters LENLWK, LENIWK, LENRWK, and LENCWK. The SPIN program checks to make sure that the user has provided enough space for the given problem. It reports how much space was allocated and how much was required. If too little space is provided, the program terminates immediately. If too much space was provided, it can be reduced by the user for the next problem. The variable NMAX sets the maximum number of mesh points that are permitted. Naturally, the larger NMAX becomes, the more working space that is required. Also, larger reaction mechanisms require more working space than smaller ones.

The boundary value solution program TWOPNT provides information about the CPU time used in the various portions of the iterations. To do so, however, it needs to make a subroutine call that is specific to the particular computer on which it is running. The user may have to change the system call in this routine to accommodate a particular computer system.

\section{PROGRAM INPUT AND OUTPUT}

The SPIN program's input is in a keyword format. On each input line an identifying keyword must appear first. For some keywords, only the keyword itself is required, while for others, additional information is required. Some keywords have default values associated with them and in such cases the keyword line need not be included. In the case of restarts or continuation problems, some of the keywords can be changed. If not changed, they retain their former values. In each keyword description, we note whether or not it can be changed upon a restart or continuation. The order in which the keywords are input is unimportant, but some keywords that appear as lists must be ordered. (For example, when entering an initial temperature profile as pairs of heights and temperatures, th.e heights are required to be in ascending order). For those keywords that affect control of the solution through interface with the TWOPNT software, the appropriate TWOPNT parameter is stated. An example keyword line is provided for all keywords. The rules goverring the syntax of the keywords are listed below:

1. The first four columns of the line are reserved for the keyword itself, which must begin in the first column.

2. Any further input associated with the keyword can appear anywhere in columns 5 through 80 . The specific column in which the information begins is unimportant. 


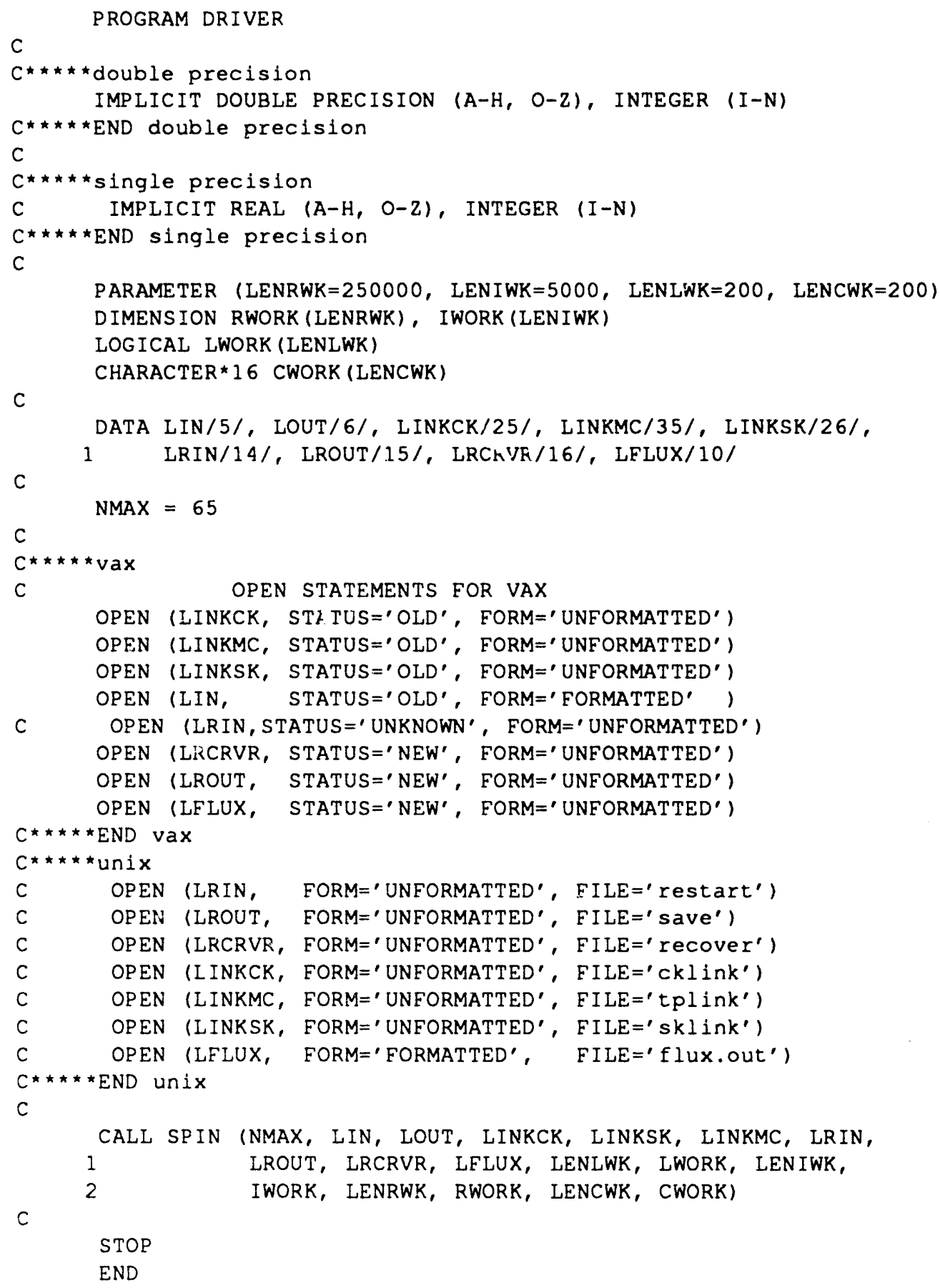

Figure 4. Example of the user-supplied main program to call the SPIN program. 
3. When the keyword requires more than one piece of data, the order in which the information appears is important. The data must be separated by one or more blank spaces.

4. When numbers are required as input, they may be stated in either integer, floating point, or $E$ format. The program converts the numbers to the proper type. The double precision specification is not recognized. If a double precision version of the program is being run, the double precision conversion is done internally.

5. When gas-phase species names are required as input, they must appear exactly as they were specified in the CHEMKIN input. When surface or bulk species names are requireri as input, they must appear exactly as they were specified in the SURFACE CHEMKIN input.

6. If keywords are repeated or conflict, then the last-read values are used. For example, if the same keyword is encountered twice, the data with the last one read is accepted.

7. A "comment" line can be inserted by placing either a period (.), a slash (/), or an exclamation point (!) in the first column. Such a line is ignored by the code, but it is echoed back in the printed output.

8. The keyword END must be the last input line.

\section{Keyword Descriptions}

\section{Problem Type}

STAG- Specify a stagnation point flow reactor. If the keyword STAG is given and the spin rate OMEG is nonzero, then this inconsistent input will generate a fatal error.

Default-stagnation point reactor is not assumed.

Example-STAG

ENRG-Inclusion of this keyword means that a solution will be obtained for the coupled energy-species equations. However, the user must still specify a temperature profile for the initial guess.

Default-none, either TGIV of ENRG must be specified.

Example-ENRG

TGIV-- Inclusion of this keyword means that a solution will be obtained using a user-specified temperature profile. The profile is specified with a sequence of TEMP keywords. An energy equation is not solved.

Default-none, either TGIV of ENRG must be specified.

Example-TGIV

RADB - This Keyword indicates that the susceptor temperature is calculated from an energy balance. If RADB is specified, TDSK is used as the initial guess for the susceptor temperature.

Default-Susceptor temperature is fixed as TDSK.

Example-RADB

RSTR - Inclusion of this keyword causes the program to begin with a previously computed solution as the starting estimate. This previously-computed solution will be rea 1 from Fortran Unit 14.

Default-solution started from input, not a restart file.

Example-RSTR

\section{Solution Method Options}

NOFT - Inclusion of this keyword means that the solution will be obtained without solving the uncoupled energy-species equations as an intermediate stage. This option is useful, for example, when starting from a previous solution, but with a new surface temperature. In this case there is no advantage to solving the species equations on the old temperature field.

Default-fixed-temperature problem solved first.

Example-NOFT 
USTG - Including this keyword on a restart means that the user-specified temperature profile, as given on the TEMP keywords, is to be used instead of the temperature profile that is on the restart file.

Default-Use the temperature profile on the restart file.

Example-USTG

\section{Newton Iteration Controls}

ATOL - Absolute tolerance for the termination of the Newton iteration. The Newton iteration is considered to be converged when the maximum norm of the solution correction vector $\Delta \phi$ is reduced to below the following criteria: $|\Delta \phi| \leq \max$ (ATOL, RTOL $\times|\phi|$ ). Typically ATOL should be smaller than the maximum mass fraction of any species of interest.

Default-1.E-9

TWOPNT Parameter-SSABS.

Example-ATOL 1.E-8

RTOL- Relative tolerance for the termination of the Newton iteration. The Newton iteration is considered to be converged when the maximum norm of the solution correction vector $\Delta \phi$ is reduced to below the following criteria: $|\Delta \phi| \leq \max ($ ATOL, RTOL $\times|\phi|)$. Typically RTOL should be in the range of $10^{-3}$ which would provide roughly three to six significant digits.

Default-1.E-4

TWOPNT Parameter-SSREL.

Example-RTOL 1.E-3

NJAC - This keyword specifies the maximum number of Newton steps that can be taken in solving the steady-state problem before a new Jacobian is evaluated. If NJAC $=1$, then a full Newton method will result.

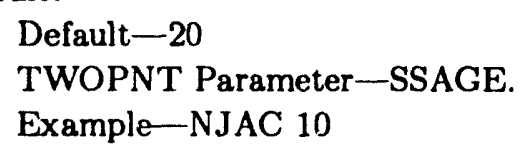

\section{Time-Step Controls}

ATIM - Absolute tolerance for the termination of the Newton iteration as it is used in the time stepping procedure. For a precise definition see the above description of ATOL. Since we are not seeking accuracy in a transient solution, this convergence criteria typically does not need to be as stringent as for the Newton iteration on the boundary value problem itself.

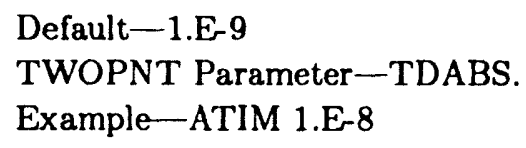

RTIM - Relative tolerance for the termination of the Newton iteration as it is used in the time stepping procedure. For a precise definition see the above description of RTOL. Since we are not seeking accuracy in a transient solution, this convergence criteria typically does not need to be as stringent as for the Newton iteration on the boundary value problem itself.

Default-1.E-4

TWOPNT Parameter-TDREL.

Example-RTIM 1.E-3 
TIME- If the Newton method fails to converge, then SPIN performs a time evolution to bring the current iterate within the domain of convergence of Newton's method. If the ISTP keyword is used, then SPIN will take initial timesteps without first attempting a Newton iteration. The TIME keyword specifies the size of the initial time step and how many time steps are to be taken. For example, the input TIME 100 1.E-6, specifies that any time Newton's method fails 100 time steps with an initial stepsize of 1 microsecond.

Units-none, seconds

Default-100, 1.E-6

TWOPNT Parameter-STEPS1, STRID0.

Example-TIME 100 1.E-5

TIM2- This keyword is the same as TIME except that TIME applies to the fixed-temperature problem and TIM2 is used after the energy equation is included. Naturally, this input only has meaning in problems that solve for a temperature profile.

Units-none, seconds

Default-100, 1.E-6

TWOPNT Parameter-STEPS1, STRID0.

Example-TIM2 100 1.E-5

UFAC- This keyword is a multiplicative factor by which the time step will be increased when appropriate (see IRET).

Units-none

Default-2.0

TWOPNT Parameter-TINC.

Example-UFAC 2.0

DFAC - This keyword is a factor by which to divide the current time step if time integration procedure fails to converge.

$$
\text { Units-none }
$$

Default-2.2

TWOPNT Parameter-TDEC.

Example-DFAC 2.5

DTMN - This keyword is the minimum time step allowed. That is, the time integration procedure will not decrease the time step below this value.

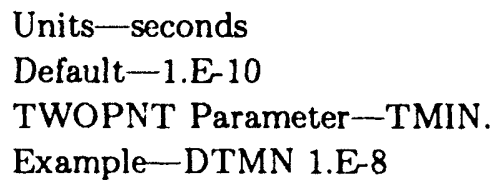

DTMX - This keyword is the maximum time step allowed. That is, the time integration procedure will not increase the time step above this value.

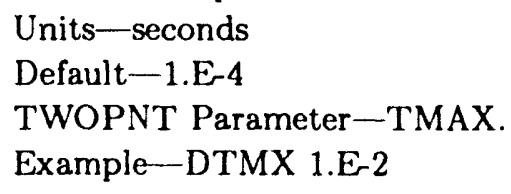

TJAC- This keyword specifies the maximum number of Newton steps that can be taken in solving the transient problem before a new Jacobian is evaluated. If $\mathrm{TJ} A C=1$, then a full Newton method will result.

Default-20

TWOPNT Parameter-TDAGE.

Example-TJAC 10 
ISTP - A positive value of this keyword specifies the number of initial time steps that are taken prior to attempting a Newton iteration. Normally, the Newton iteration will be attempted first, with time steps invoked only if the Newton iteration fails. Nevertheless, there may be circumstances where initial time stepping is desirable. The time step size is specified with the TIME keyword. The ISTP Keyword only applies to the first grid network, not the subsequently refined ones. If ISTP has a negative value (e.g., ISTP - 100 ), then no Newton steps will be attemped. Invoking this option would be unusual, but could be used to find a steady-state solution via pure time integration.

Default-0

TWOPNT Parameter--STEPSO and STEADY.

Example-ISTP 200

IRET- This keywcrd specifies the number of time steps to be taken before the time step value is increased by the factor UFAC.

Default-50

TWOPNT Parameter-STEPS2.

Exampie-IRET 100

\section{Solution Bounds Controls}

SFLR - Sometimes during the solution procedure some of the very small gas-phase mass fractions, surface site fractions, or bulk species fractions may be calculated as negative. No solution component will be allowed to drop below the floor value specified by SFLR.

Default- -1.5-4

TWOPNT Parameter-BELOW.

Example-SFLR -1.E-5

SPOS- This Keyword provides a (small positive) number that will replace any negative species mass fractions. The replacement is made after every successful sequence of time steps, upon adding mesh points, and on restart or continuation. SPOS is often helpful in starting difficult problems when the initial guess is far from the solution. SPOS attempts to force the transient solution away from nonphysical regions that may otherwise be entered due to $r$ d initial conditions and badly conditioned systems.

Default-does not apply.

Example-SPOS 1.E 9

\section{Grid Control Parameters}

NPTS - The number of initial mesh points. The inclusion of NPTS will generate an equispaced mesh of NPTS points between XSTR and XEND. The user can also specify an initial nonuniform mesh using the keyword GRID, in which case the NPTS input is not needed.

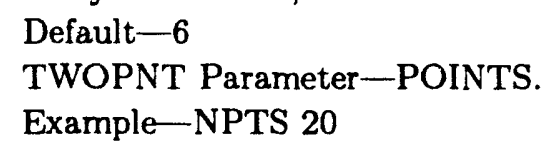

NMAX - The maximum number of mesh points allowed for this solution. The adaptive gridding will stop when this number of grid points has been reached.

Default-NTOT, a dimension supplied by the Driver program.

TWOPNT Parameter-PMAX.

Example-NMAX 50 
NADP - The maximum number of mesh points allowed to be added at each step in the grid adaption process. Occasionally the program has trouble converging when adding too many points at once. This keyword will limit the number of new points added before a new solution will be found.

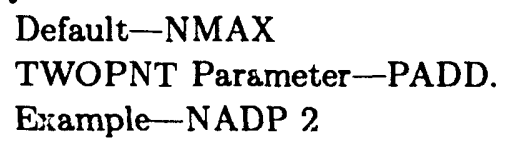

JJRG-- On continuations or restarts, the number of mesh points can be reduced. TWOPNT itself does not remove grid points. Therefore, on a sequence of continuation problems the number of grid points can grow because the region where they are needed may change. SPIN thus provides a capability to remove grid points. JJRG is the reduced number of grid points that will be used. The old solution is adaptively interpolated onto a new grid of JJRG points.

Default-no grid reduction; old grid used.

Example-JJRG 40

PCAD - On continuations or restarts the number of mesh points can be reduced. PCAD is a parameter that influences the fraction of grid points that are dedicated to adaption. PCAD of zero would result in a uniform grid. The old solution is adaptively interpolated onto a new grid of JJRG points.

Default- 0.75

Example-PCAD .5

RGTC- On continuaions or restarts the number of mesh points can be reduced. RGTC is a parameter that influences the ratio of gradient regrid points to curvature regrid points. RGTC of zero would cause all regrid points dedicated to adaption to be used to equidistribute curvature in the old solution. The old solution is adaptively interpolated onto a new grid of JJRG points.

Default-1.0

Example-RGTC 1

GRID- This optional keyword allows the user to input an initial grid. Up to NMAX of these GRID inputs can be included. Each GRID input provides the coordinate of a mesh point. They must appear in ascending order. If this keyword is not included, the grid will assumed to be equispaced and based on NPTS.

Units-cm

Default-equally spaced grid.

Example-GRID 0.1

GRAD - Adaptive mesh parameter that controls the number of grid points inserted in regions of high gradient. Smaller values of GRAD cause more grid points to be used. See Eq. (32).

Default-0.1

TWOPNT Parameter-TOLER1.

Example-GRAD .2

CURV- Adaptive mesh parameter that controls the number of grid points inserted in regions of high curvature. Smaller values of CURV cause more grid points to be used. See Eq. (33).

Default- 0.5

TWOPNT Parameter-TOLER2.

Example-CURV 0.5

Initial-Guess and Reactor Operating Conditions

PRES- The pressure.

Units-atmospheres

Default--PRES is a required input.

Example-PRES 0.5 
REAC- Mole fraction values of the reactants entering at the inlet. One of these REAC inputs must appear for each reactant species, for example "REAC SIH4 0.5." The sum of all the reactant mole fractions should equal 1 . However, if they do not, a cautionary message will be printed and the mole fractions will be normalized so the sum does equal one. Any given species can be specified simultaneously as a reactant, intermediate, or product.

Units-none, mole fractions

Default-required input.

Example-REAC H2 0.3

INTM - The estimated peak mole fractions values for "intermediate" species. Providing one of these INTM inputs for each intermediate species often aids in the convergence. It is usually better to estimate values somewhat higher than those that are actually present in the gas. Any given species can be specified simultaneously as a reactant, intermediate, or product.

Units-none, mole fractions.

Default- 0.0

Example-INTM SIH2 1.E-5

PROD-Estimated values of the gas-phase mole fractions at the deposition surface. One of these PROD inputs should appear for each species in large concentration at the surface. The sum of the product mole fractions should equal 1 . However, if they do not, a cautionary message will be printed and the mole fractions will be normalized so the sum does equal one. Any given species can be specified simultaneously as a reactant, intermediate, or product.

Units-none, mole fractions

Default-required input.

Example-PROD SIH4 0.1

SURF-..- Iface site fraction values estimated for the surface species on each surface site type (surface phase) on the deposition surface. One of these SURF inputs should appear for each surface species that is expected to be in large concentration on the surface. The sum of the site fractions should equal 1 for each surface site type (surface phase). However, if they do not, a cautionary message will be printed and the site fractions for each surface site type will be normalized so that the sum does equal 1.

Units-none, surface site fractions

Default-required input.

Example-SURF SI(S) 1.E-2

ACT - Estimated activitites for the bulk species in each bulk phase. One of these ACT inputs should appear for each species in a bulk phase. For an ideal bulk-phase mixture the sum of the bulk species activities should equal 1 . However, if they do not, a cautionary message will be printed and the bulk species fractions for each bulk phase will be normalized so the sum does equal 1.

Units-none, bulk species fractions

Default-required input.

Example-ACT SI(B) 1.0

TEMP - This input allows specification of the initial guess or given temperature profile. Each input provides an $(x, T)$ pair and the $x$ coordinates must be in ascending order. Up to NMAX $(x, T)$ pairs may be input. Example: TEMP 0.11000.

Units- $\mathrm{cm}, \mathrm{K}$

Default-required input, except on a restart.

Restart-can be changed, provided USTG is included.

Example-TEMP 0.11540 . 
TINF - Inlet gas temperature, i.e., temperature at XEND.

Units-K

Default-TINF is required input.

Example-TINF 1000.

TDSK - Temperature of the deposition surface. This is a constant value taken as a boundary condition, unless the keyword RADB is given, indicating that the susceptor temperature is calculated from an energy balance. If RADB is specified, TDSK is taken as the initial guess for the susceptor temperature.

Units-K

Default-TDSK is required input.

Example-TDSK 1200.

OMEG-The disk rotation rate. A stagnation-point flow can be specified either by using OMEG 0 , or by using the keyword STAG.

Units-rpm

Default-OMEG is required input, unless the STAG option is used

Example-OMEG 1000

AINL - The radial velocity spreading rate. At the inlet $x=L, v / r=$ AINL.

Units- $1 /$ sec

Default-0.0

Example-AINL 2.3

OINL- The inlet-gas spin rate. At the inlet $x=L, w / r=$ OINL.

Units-rpm

Default -0.0

Example-OINL 100

UINF - The axial inlet velocity at position XEND to be specified as a boundary condition. In the usual rotating-disk solution, the velocity at the outer domain is calculated as a part of the solution. However, using the keyword UINF, one may impose this velocity upon the solution. If the rotation rate OMEG is 0 , or if the STAG option is used, then UINF is no longer optional, but must be given to specify the problem.

Units- $\mathrm{cm} / \mathrm{sec}$

Default-none, UINF is required input for stagnation-point flow.

Example-UNIF 23.6

XSTR - The beginning of the computational interval.

Units-cm

Default- -0.0

Restart-can be changed to a smaller value.

Example-XSTR 0.0

$\mathrm{XCEN}-\mathrm{An}$ estimated value for center of the reaction boundary layer.

Units-cm

Default-XCEN $=0.35$ (XEND-XSTR)

Example-XCEN 1.8

XEND - The end of the computational interval. That is, the inlet position above the disk.

Units-cm

Default-XEND is a required input.

Restart-can be changed to a larger value.

Example-XEND 5.0 
WMIX - Estimated width of the reaction boundary layer.

$$
\begin{aligned}
& \text { Units-cm } \\
& \text { Default-WMIX }=0.5 \text { (XEND-XSTR) } \\
& \text { Example-WMIX } 1.0
\end{aligned}
$$

TWAL - Temperature of a neighboring "wall" used in calculating a surface radiation balance. This value is used only if the disk temperature is being calculated. See Eq. (17).

$$
\begin{aligned}
& \text { Units-K } \\
& \text { Default-500 K } \\
& \text { Example-TWAL } 850.0
\end{aligned}
$$

EMIS- The emissivity of the disk. This value is used only if the disk temperature is being calculated from an energy balance. See Eq. (17).

$$
\begin{aligned}
& \text { Units-none } \\
& \text { Default-0.85 } \\
& \text { Example-EMIS } 0.9
\end{aligned}
$$

POWR-The power being supplied to heat the disk. This value is used only if the disk temperature is being calculated from an energy balance. See Eq. (17).

$$
\begin{aligned}
& \text { Units-ergs } / \mathrm{cm}^{2} / \mathrm{sec} \\
& \text { Deiault-0.0 } \\
& \text { Example-POWR 15.E7 }
\end{aligned}
$$

QDOT - The power being supplied to heat the gas as a spatially distributed Gaussian heat source. QDOT is the total power, i.e., the spatial integral of the heat-source function. See Eq. (13).

$$
\text { Units-ergs } / \mathrm{cm}^{2} / \mathrm{sec}
$$

Default- 0.0

Example-QDOT 1.0E7

XSRC- - Height above the disk which is the center of a Gaussian-shaped power source. See Eq. (12).

Units-cm

Default-0.0, XSRC is required input if QDOT is nonzero.

Example-XSRC 0.6

WSRC-Half-width of the Gaussian source term if the gas is being heated by an optional heat source. See Eq. (12).

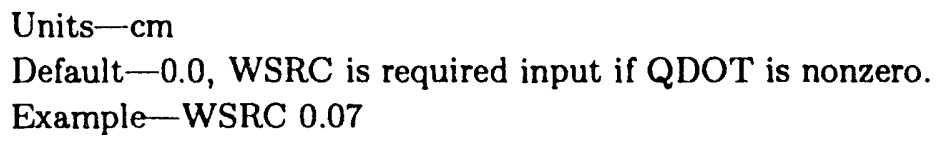

\section{Multicomponent Transport Options}

MULT - Including this keyword causes the calculation to be run with a full multicomponent model for the transport coefficients and diffusion fluxes.

Default-mixture-averaged, not multicomponent, transport is used.

Example-MULT

MIX - Including this keyword causes the calculation to be run using a mixture-average model for calculating the transport coefficients and diffusion fluxes.

Default-mixture-average transport is used.

Example-MIX

TDIF- Including this keyword causes the calculation to be run including thermal diffusion (Soret effect). The thermal diffusion coefficients are always calculated from the multicomponent modei. However, the keywords MULT or MIX still determine whether the diffusion coefficients and diffusion velocities are calculated with the multicomponent model.

Default-no thermal diffusion. 
VCOR - Including this keyword causes the calculation to be run using a correction velocity to ensure mass conservation, i.e., the sum of the diffusion fluxes is zero. See Eq. (11). If VCOR is not used, then TRCE is in effect.

Default-a correction velocity is not used.

Example-VCOR

TRCE- Including this keyword causes the calculation to be run by setting the mass fraction of the lastnamed CHEMKIN gas-phase species to be 1 minus the sum of the mass fractions of the other species. A conservation equation is not solved for the last species.

Default-TRCE is used.

Example-TRCE

REOR - The option may be used when the TRCE option is in effect. Using TRCE, the conservation equation for the last species in the the gas phase and each surface and bulk phase is not solved: the last species concentration (mass fraction or site fraction) is chosen so that the fractions sum to 1 . The REOR option causes TRCE to choose dynamically and locally (at each mesh point and in each phase) the species of largest concentration and not solve its conservation equation.

Default-The last species in each phase is chosen.

Example-REOR

\section{Sensitivity Options}

ASEN - Including this keyword causes all the first-order sensitivity coefficients with respect to the reaction rate constants to be determined.

Default-no sensitivities computed.

Example-ASEN

HSEN-- Including this keyword causes all the first-order sensitivity coefficients with respect to the species heats of formation to be determined.

Default-no sensitivities computed.

Example-HSEN

\section{Chemistry Control Options}

NONR-This keyword specifies that the nonreacting problem will not be solved as the first stage in the solution of the full problem.

Default-The nonreacting problem is solved first.

Example-NONR

NOCH - This keyword specifies that the rates of all gas-phase reactions will be set to zero, no matter what the values specified in the CHEMKIN input.

Default-actual values for chemistry rates are used.

Example--NOCH

CHEM - This keyword specifies that the rates of all gas-phase reactions will be calculated. This option is used to reactivate the chemical kinetics if the NOCH option was in effect for the previous calculation.

Default-actual values for chemistry rates are used

Example-CHEM

GFAC- This keyword specifies that the rates of all gas-phase reactions will be multiplied (scaled) by the factor GFAC. This option is sometimes useful if convergence difficulties are encountered due to unusually large reaction rates. The problem would be first solved with artificially reduced reaction rates, which are increased in subsequent continuations or restarts until GFAC is 1.

Default -1.0

Example--GFAC 0.3 
SFAC- This keyword specifies that the rates of all surface reactions will be multiplied (scaled) by the factor SFAC. This option is sometimes useful if convergence difficulties are encountered due to unusually large reaction rates. The problem would be first solved with artificially reduced reaction rates, which are increased in subsequent continuations or restarts until SFAC is 1 . SFAC can be a small number, but it cannot be exactly zero.

Default-1.0

Example-SFAC 0.3

\section{Printing Options}

PRNT - Printing control. "PRNT 1" provides less printed output of intermediate solutions than does "PRNT 2." More printing is often helpful when there are convergence difficulties.

Default-1

TWOPNT Parameter-LEVELD and LEVELM.

Example-PRNT 1

LPRT - Printing control. LPRT turns on extensive printing that provides information on rates of progress of individual surface reactions. This can be very informative in understanding the surface reaction behavior.

Default-LPRT not invoked

Example-LPRT

\section{Miscellaneous}

FILE- A restart file may contain more that one solution. Specifying the keyword FILE causes the program to select the specified solution number from a restart file. For example, "FILE 3" means that the first two solutions on the restart file should skipped and the next one used. If solutions have already been read from a restart file, the number of the solution to use starts from its current position. The FILE keyword is valid only if the RSTR keyword is also used.

Default-if RSTR was specified, the first solution will be used.

Example-FILE 3

CNTN - Including this keyword causes the program to expect keywords for another problem following the END keyword. The following problem will use the solution of the previous problem as its initial guess. This capability is very similar to that provided by RSTR. However, in the case of CNTN, several related problems can be solved by one job submission without having to manipulate the restart files. Any keyword that can be changed for a RSTR problem can be changed on continuation.

Default-no continuation is expected.

Example-CNTN

END- This line signifies the end of a given set of input data. It must appear after each set of input when continuation jobs are indicated using the CNTN keyword.

Example-END 
If a sensitivity calculation was performed by SPIN, the sensitivity coefficients appear next. For each of the II gas-phase reactions we read the sensitivity coefficients for: the surface site fractions, the NATJ components of the gas-phase solution at each node, and the production rates of the bulk-phase species. Following that, for each of the IISUR surface reactions we read the sensitivity coefficients for same set of surface, bulk and gas-phase components of the solution.

The file LROUT (Unit 15) is written after the solution to a problem is complete. However, another file LRCRVR (Unit 16) is rewound and written after every successful return from a Newton iteration or a sequence of time steps. In the event that the code fails to complete a problem for some reason it may be possible to restart it from the LRCRVR file. Starting from LRCRVR, but with different keyword inputs (for example a different time step) may be more efficient than restarting the problem from the beginning.

We find it quite useful to artificially create restart files for a given deposition from the converged solution of a "related" deposition. For example, we could be interested in the solution of a GaAs deposition using a reaction mechanism involving a detailed treatment of the gas-phase hydrocarbon intermediates. However, we could start the process by solving the same deposition problem using a much simpler reaction mechanism including only surface reactions. We then write a program that reads the CHEMKIN Linking files for both mechanisms and the restart file from the first problem. The second problem of course contains intermediate species that were not present in the first problem. A new file is written that has the solutions for the species that are in the shorter mechanism and zeros for those species that are only in the second mechanism. In this way we can often save computer time in starting new problems. Small programs of this type to manipulate restart files can often be written to save computer time for certain applications.

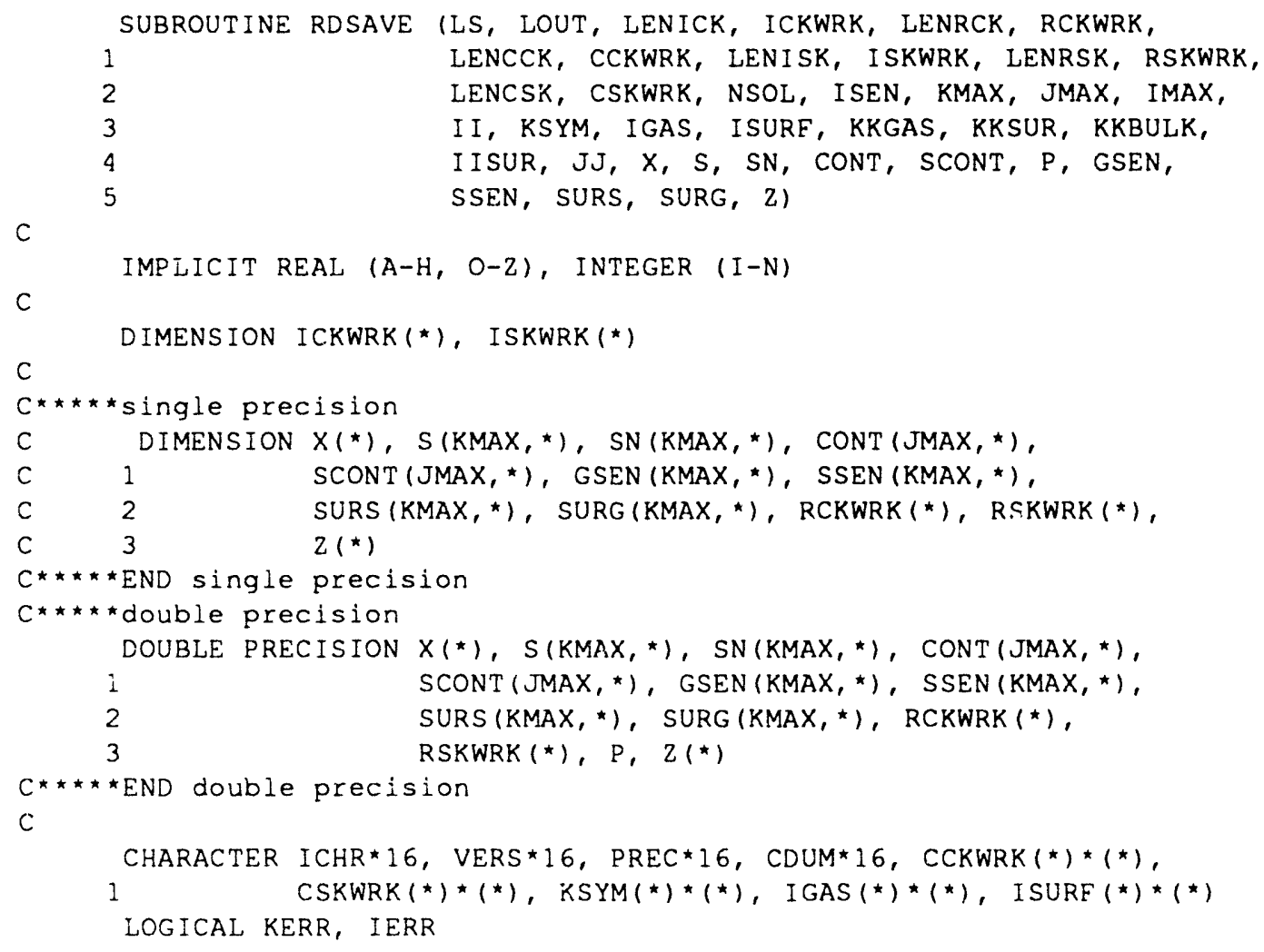




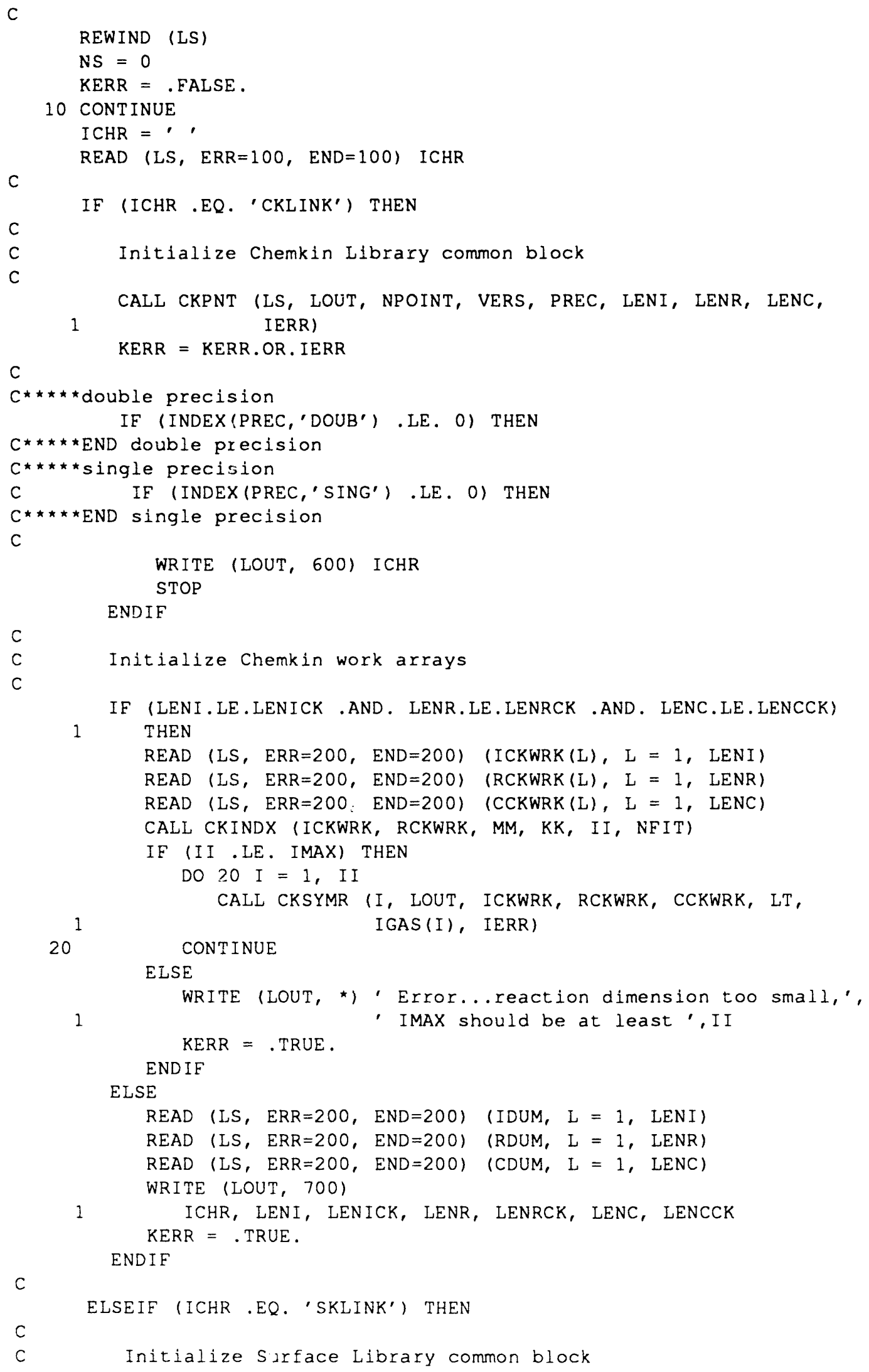


CALL SKPNT (LS, LOUT, NPOINT, VERS, PREC, LENI, LENR, LENC,

1 IERR)

$\mathrm{C}$

$K E R R=K E R R \cdot O R \cdot I E R R$

$C \star \star \star \star \star$ double precision

IF (INDEX (PREC,'DOUB') .LE. O) THEN

$C \star \star \star \star \star$ END double precision

$C \star \star \star \star \star$ single precision

C IF (INDEX (PREC,'SING') .LE. O) THEN

$C \star \star \star \star \star E N D$ single precision

C

WRITE (LOUT, 600) ICHR

STOP

ENDIF

C

Initialize surface chemistry work arrays

IF (LENI.LE.LENISK . AND. LENR.LE.LENRSK .AND. LENC.LE.LENCSK)

1 THEN

READ (LS, $E R R=200, E N D=200) \quad(\operatorname{ISKWRK}(L) ; L=1, L E N I)$

READ (LS, ERR $=200, E N D=200) \quad(R S K W R K(L), L=1, L E N R$ )

READ (LS, $E R R=200, E N D=200) \quad(\operatorname{CSKWRK}(L), L=1, L E N C)$

CALI SKINDX (NELEM, KKGAS, KKSUR, KKBULK, KKTOT, NPHASE,

1 NNSURF, NFSURF, NLSURF, NNBULK, NFBLLK,

2 NLBULK, IISUR)

IF (KKTOT .LE. KMAX) THEN

CALL SKSYMS (CSKWRK, LOUT, KSYM, IERR)

ELSE

1

WRITE (LOUT, *) 'Error...species dimension too small,', KERR $=$. TRUE.

ENDIF

IF (IISUR . LE. IMAX) THEN

DO 30 IR $=1$, IISUR

CALI SKSYMR (IR, LOUT, ISKWRK, RSKWRK, CSKWRK, LY,

1

30

CONTINUE

ELSE

1

WRITE (LOUT, *) 'Error...reaction dimension too small,',

- IMAX should be at least ', I ISUR ENDIF

ELSE KERR $=$. TRUE.

READ (LS, $E R R=200, E N D=200$ ) (IDUM, L $=1$, LENI)

READ (LS, $E R R=200, E N D=200$ ) (RDUM, L $=1$, LENR)

READ (LS, $E R R=200, E N D=200$ ) (CDUM, L $=1$, LENC)

WRITE (LOUT, 700)

1

ICHR, LENI， LENICK， LENR，LENRCK，LENC， LENCCK

ENDIF

C

ELSEIE (ICHR .EQ. 'MCLINK') THEN

Initialize Transport Library common block

CALL MCDNT (LS, LOUT, NPOINT, VERS, PREC, LENI, LENR, IERR)

KERR $=$ KERR. OR. IERR 


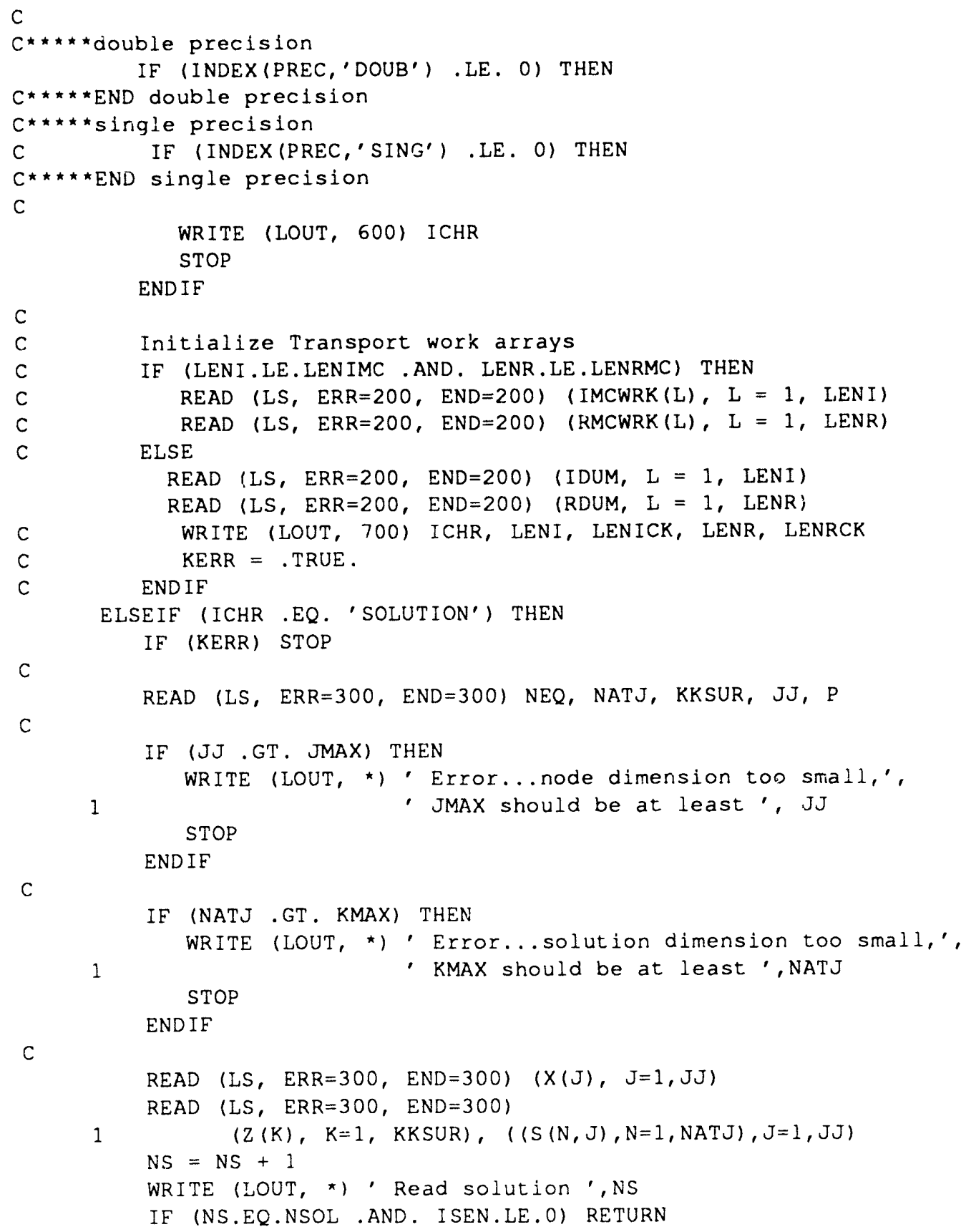


C

ELSEIF (ICHR .EQ. 'SENSITIVITY') THEN

DO $40 I=1$, II

READ (LS, ERR $=400$, END $=400$ ) IS, ( $S U R G(K, I), K=1, K K S U R$ ),

1

$((\mathrm{SN}(\mathrm{N}, \mathrm{J}), \mathrm{N}=1, \mathrm{NAT} J), \mathrm{J}=1, \mathrm{JJ})$

READ (LS, ERR $=400, E N D=400$ ) IS, (GSEN $(K, I), K=1, K K B U L K$ )

IF (ISEN .GT. O) THEN

DO $35 \mathrm{~J}=1$, J J

$\operatorname{CONT}(J, I)=\operatorname{SN}(I S E N, J)$

35 CONT INUE

ENDIF

40 CONTINUE

DO $50 I=1$, IISUR

READ (LS, $E R R=500, E N D=500$ ) IS, ( $\operatorname{SURS}(K, I), K=1, K K S U R$ ),

1

$((\mathrm{SN}(\mathrm{N}, \mathrm{J}), \mathrm{N}=1, \mathrm{NAT} J), \mathrm{J}=1, \mathrm{JJ})$

READ (LS, ERR $=500, E N D=500)$ IS, (SSEN (K,I), K=1, KKBULK)

IF (ISEN .GT. O) THEN

DO $45 \mathrm{~J}=1$, JJ

$\operatorname{SCONT}(J, I)=\operatorname{SN}(I S E N, J)$

45 CONTINUE

ENDIF

50 CONTINUE

WRITE (LOUT, *) ' Read sensitities for solution',NS

IF (NS .EQ. NSOL) RETURN

ENDIF

GO TO 10

C

100 CONTINUE

WRITE (LOUT, 110) ICHR

110 FORMAT ('Error reading ', A ,' record')

STOP

C

200 CONTINUE

WRITE (LOUT, 210) ICHR

210 FORMAT ('Error reading ', A, ' data...')

STOP

C

300 CONTINUE

WRITE (LOUT, *) ' Error reading solution...'

WRITE (LOUT, *) ' NEQ=', NEQ,', NATJ=', NATJ,', KKSUR=', KKSUR,

1

', JJ=', JJ, ', P=', P,', NSOL=', NSOL,', NS=' , NS

STOP

C

400 CONTINUE

WRITE (LOUT, *) ' Error reading gas-phase sensitivities...'

WRITE (LOUT, *) ' ISEN=', ISEN,', NSOL=', NSOL,', NS=', NS,

1

, II $=$ ', II,', I=', I

STOP

500 CONTINUE

WRITE (LOUT, *) ' Error reading surface-phase sensitivities...'

WRITE (LOUT, *')' ISEN=', ISEN,', NSOL=', NSOL,', NS=', NS,

1

', IISUR=' , I ISUR,' , I=' , I

STOP 
C

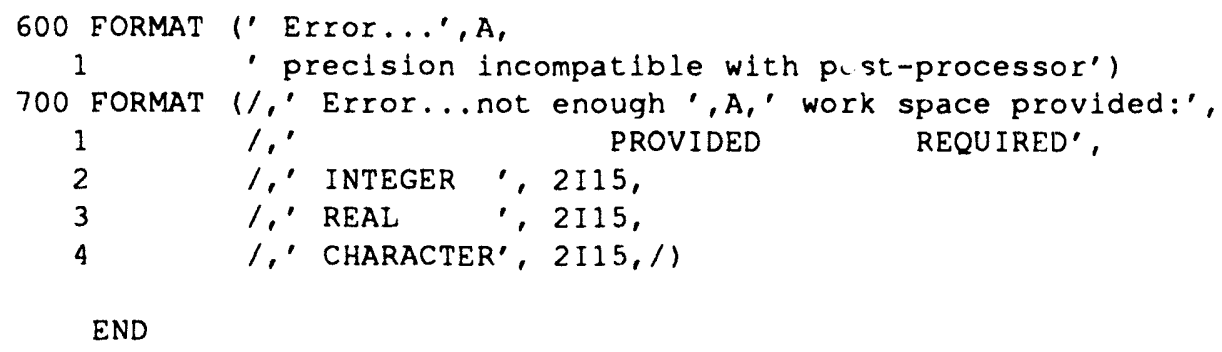

\section{SAMPLE PROBLEM}

This section shows an example using the SPIN code. All sample input and output files are included.

We have chosen as a sample the deposition of $\mathrm{Si}_{3} \mathrm{~N}_{4}$ from $\mathrm{SiF}_{4}$ and $\mathrm{NH}_{3}$. The gas-phase reaction mechanism contains a detailed description of $\mathrm{NH}_{3}$ decomposition (about which there is much published information), two reactions describing $\mathrm{SiF}_{4}$ decomposition, and three cross reactions. At the low pressures we consider, the gas-phase decomposition of reactants is slow. The surface reaction mechanism contains six steps describing the overall conversion of $3 \mathrm{SiF}_{4}$ and $4 \mathrm{NH}_{3}$ molecules to $3 \mathrm{Si}(\mathrm{d})$ and $4 \mathrm{~N}(\mathrm{~d})$ and $12 \mathrm{HF}$. (Note that the surface reaction mechanism is from a preliminary analysis at one temperature, and thus we have not supplied any activation energies. As such, this mechanism should only be considered as illustrative and not as a source of kinetic data on the $\mathrm{Si}_{3} \mathrm{~N}_{4}$ system.)

The first file is the input file for the CHEMKIN Interpreter, which defines the gas-phase reaction mechanism. The output from the CHEMKIN Interpreter follows. The input to and output from the SURFACE CHEMKIN Interpreter show the surface species and surface reactions in the problem. More details on the CHEMKIN and SURFACE CHEMKIN Interpreters can be found in the user's manuals for each code. ${ }^{13,15}$

The file containing the keyword input to SPIN specifies the specific run conditions for the problem: the energy equation (ENRG), rotation rate of $1000 \mathrm{rpm}$ (OMEG), disk temperature $1713 \mathrm{~K}$ (TDSK), inlet temperature $300 \mathrm{~K}$ (TINF), 11 mesh points in the initial grid (NPTS), the computational domain ends at 10 $\mathrm{cm}(\mathrm{XEND})$, and pressure of $2.3684 \times 10^{-3}$ atm (PRES). The reactant mole fractions at the inlet are given (REAC), along with estimates of the major product concentrations at the surface (PROD), site fractions of the surface species (SURF), and activities of the bulk phase species (ACT). A crude temperature profile to begin the iteration is specified (TEMP). The remaining keywords specify numerical error tolerances, etc.

The last file is the output from the SPIN code. A series of banners shows the version numbers of SPIN and the CHEMKIN, SURFACE CHEMKIN, and TRANSPORT libraries. After giving statistics on the working space requirements, the code echoes back the keyword inputs. The code first solves the nonreacting problem, calculating the temperature and velocity fields. The first thing printed is the initial guess at the solution, which is based on the keyword inputs.

The numerical two-point boundary-value solver TWOPNT actually solves the problem. TWOPNT reports the progress of its solution as part of the rinted output. Since the PRNT keyword was set to 1, only brief statistics for the progress of the iteration are provided. There are three major functions in the code, and each reports what it has done at each stage of the solution. These functions are NEWTON (which attempts to solve the boundary value problem on the given mesh by Newton's method), TIMSTP (which takes the user-specified time steps if Newton's method fails), and REFINE (which performs the mesh adaptation). The first column reports the logarithm of the maximum residual norm $\|F(\phi)\|$. The second column reports the logarithm of the largest Jacobian condition number encountered during the current iteration. These condition numbers are obtained from the LINPACK condition number estimates. The next column reports 
the number of mesh points, the number of Newton or time steps, and the number of Jacobian evaluations. The last column provides a remark about the given operation. For example, the "FAILURE" remarks would indicate that the Newton method failed to converge and thus time steps would be attempted next. Since the nonreacting problem is quite easy to solve there is little diagnostics coming from TWOPNT. When TWOPNT has solved the problem it reports the solution and gives statistics on the amount of time required for various parts of the computation.

Next, SPIN includes the gas-phase species continuity and surface species equations, although the energy equation is omitted. The temperature and velocity fields in the initial guess are from the nonreacting solution just obtained. The initial species fractions are set from the user's keyword input. TWOPNT reports that the $\log$ of the solution's residuals was reduced by 5.2 during the Newton iteration. Again, the Newton interation had no trouble solving the problem, and no time stepping was required. The final solution to the fixed temperature problem is then printed.

The solution from this calculation is used as input to the final one, which now turns on the energy equation to solve the fully coupled problem. Note that now the initial guess should be very close solution and the Newton procedure converges rapidly. At this stage in the calculation, the code begins to adaptively add mesh points in order to resolve the solution to the accuracy specified on input. The final solution is calculated on a mesh of 34 grid points. 


\section{Input to CHEMKIN Interpreter for the Example}

ELEMENTS H N SI F

END

SPECIES

H2 H N2 N NH NH2 NNH N2H2 N2H3 N2H4

HF F SIF4 SIF3 SIHF 3 SIF 3 NH2

NH3

END

THERMO

SI

J 3/67SI 1000000 0000 OOG $300.000 \quad 5000.000$

$0.26506014 \mathrm{E} \quad 01-0.35763852 \mathrm{E}-03 \quad 0.29592293 \mathrm{E}-06-0.72804829 \mathrm{E}-10 \quad 0.57963329 \mathrm{E}-14$

$\begin{array}{lllllllll}0.53437054 \mathrm{E} & 05 & 0.52204057 \mathrm{E} & 01 & 0.31793537 \mathrm{E} & 01-0.27646992 \mathrm{E}-02 & 0.44784038 \mathrm{E}-05\end{array}$

$\begin{array}{lllllll}-0.32833177 \mathrm{E}-08 & 0.91213631 \mathrm{E}-12 & 0.53339032 \mathrm{E} & 05 & 0.27273204 \mathrm{E} & 01 & 0.00000000\end{array}$

$\begin{array}{lllllllll}\text { SIF2 } & 41889 S I & 1 F & 2 & 0 & \text { OG } & 300.000 & 3000.000 & 1000.00\end{array}$

$0.61424704 \mathrm{E}+01 \quad 0.78079745 \mathrm{E}-03-0.13393120 \mathrm{E}-06-0.62648393 \mathrm{E}-10 \quad 0.17251383 \mathrm{E}-13$

$-0.77440422 E+05-0.47123275 E+01 \quad 0.38453453 E+01 \quad 0.60384651 E-02-0.11677322 E-05$

$-0.45795536 \mathrm{E}-08 \quad 0.26074143 \mathrm{E}-11-0.76816336 \mathrm{E}+05 \quad 0.72 \% 29836 \mathrm{E}+01$

$\begin{array}{lllllllll}\text { SIF } & 41889 S I & 1 F & 1 & 0 & \text { OG } & 300.000 & 3000.000 & 1000.60\end{array}$

$0.41200666 \mathrm{E}+01 \quad 0.35488207 \mathrm{E}-03-0.72002223 \mathrm{E}-07-0.21904345 \mathrm{E}-10 \quad 0.67645906 \mathrm{E}-14$

$\begin{array}{lllll}-0.75613784 \mathrm{E}+04 & 0.27842460 \mathrm{E}+01 & 0.31449478 \mathrm{E}+01 & 0.25885573 \mathrm{E}-02-0.57959124 \mathrm{E}-06\end{array}$

$-0.18072788 \mathrm{E}-08 \quad 0.10411718 E-11-0.72944390 \mathrm{E}+04 \quad 0.78767738 \mathrm{E}+01$

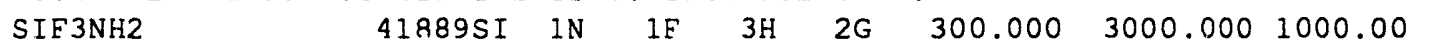

$0.12109636 \mathrm{E}+02 \quad 0.43832823 \mathrm{E}-02-0.41422453 \mathrm{E}-06-0.39890902 \mathrm{E}-090.89589543 \mathrm{E}-13$

$-0.16417678 \mathrm{E}+06-0.30469284 \mathrm{E}+02 \quad 0.62294030 \mathrm{E}+01 \quad 0.17780151 \mathrm{E}-01-0.26123043 \mathrm{E}-05$

$-0.12672435 \mathrm{E}-070.70445559 \mathrm{E}-11-0.16258489 \mathrm{E}+06 \quad 0.20454407 \mathrm{E}+00$

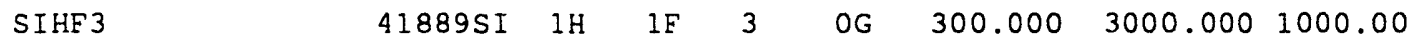

$0.93635674 \mathrm{E}+01 \quad 0.29475559 \mathrm{E}-02-0.35776330 \mathrm{E}-06-0.28582245 \mathrm{E}-09 \quad 0.69157286 \mathrm{E}-13$

$-0.14860736 E+06-0.21694529 E+02 \quad 0.3918 C: 29 E+01 \quad 0.14639172 E-01-0.18560698 E-05$

$-0.10582003 E-070.56175433 E-11-0.14704386 E+06 \quad 0.70242615 E+01$

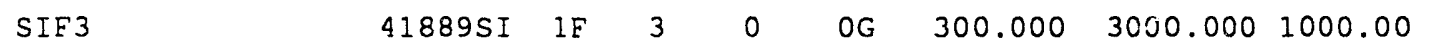

$0.85247898 E+01 \quad 0.13237924 E-02-0.21042787 E-06-0.11495040 E-09 \quad 0.30553014 E-13$

$-0.12235223 \mathrm{E}+06-0.15502343 \mathrm{E}+02 \quad 0.46628685 \mathrm{E}+01 \quad 0.10087878 \mathrm{E}-01-0.18055442 \mathrm{E}-05$

$-0.77692990 \mathrm{E}-08 \quad 0.43778518 \mathrm{E}-11-0.12129652 \mathrm{E}+06 \quad 0.46729660 \mathrm{E}+01$

$\begin{array}{lllllll}\text { SIE4 J } 6 / 76 S I & 1 F & 4 & 0 & \text { OG } & 300.000 & 5000.000\end{array}$

$0.10478473 \mathrm{E} \quad 02 \quad 0.28586756 \mathrm{E}-02-0.12646314 \mathrm{E}-05 \quad 0.24746863 \mathrm{E}-09-0.17824296 \mathrm{E}-13$

$\begin{array}{llllll}-0.19790550 \mathrm{E} & 06-0.27520641 \mathrm{E} & 02 & 0.21893068 \mathrm{E} & 01 & 0.33702007 \mathrm{E}-01-0.46723179 \mathrm{E}-04\end{array}$ $0.31584638 \mathrm{E}-07-0.84506114 \mathrm{E}-11-0.19603289 \mathrm{E} \quad 060.13287308 \mathrm{E} \quad 02 \quad 0.00000000$

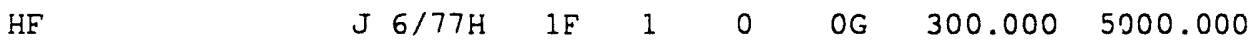

$0.29919110 \mathrm{E}$ OI $0.71489475 \mathrm{E}-03-0.68630973 \mathrm{E}-07-0.11617130 \mathrm{E}-10 \quad 0.19412375 \mathrm{E}-14$

$\begin{array}{llllllll}-0.33621364 \mathrm{E} & 05 & 0.38123288 \mathrm{E} & 01 & 0.34379986 \mathrm{E} & 01 & 0.53571598 \mathrm{E}-03-0.15229655 \mathrm{E}-05\end{array}$ $0.17564491 \mathrm{E}-08-0.57869940 \mathrm{E}-12-0.33818972 \mathrm{E} \quad 050.11930153 \mathrm{E} \quad 01 \quad 0.00000000$

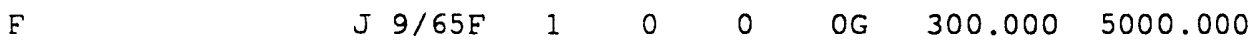

$0.27004353 \mathrm{E} \quad 01-0.22293182 \mathrm{E}-03 \quad 0.97941385 \mathrm{E}-07-0.19123038 \mathrm{E}-10 \quad 0.13768154 \mathrm{E}-14$ $0.87163617 \mathrm{E} \quad 04 \quad 0.38067182 \mathrm{E} \quad 01 \quad 0.28128740 \mathrm{E} \quad 01-0.33023098 \mathrm{E}-05-0.12897310 \mathrm{E}-05$ $0.16837365 \mathrm{E}-08-0.64587833 \mathrm{E}-12 \quad 0.86604019 \mathrm{E} \quad 04 \quad 0.30984198 \mathrm{E} \quad 01 \quad 0.00000000$ END 
REACT IONS

$\mathrm{H}+\mathrm{H}+\mathrm{M}=\mathrm{H} 2+\mathrm{M}$

$\mathrm{H} 2 / 0.0 /$

$\mathrm{H}+\mathrm{H}+\mathrm{H} 2=\mathrm{H} 2+\mathrm{H} 2$

$\mathrm{NH}+\mathrm{N}=\mathrm{N} 2+\mathrm{H}$

$\mathrm{NH}+\mathrm{H}=\mathrm{N}+\mathrm{H} 2$

$\mathrm{NH} 2+\mathrm{H}=\mathrm{NH}+\mathrm{H} 2$

$\mathrm{NH} 3+\mathrm{H}=\mathrm{NH} 2+\mathrm{H} 2$

$\mathrm{NNH}=\mathrm{N} 2+\mathrm{H}$

$\mathrm{NNH}+\mathrm{H}=\mathrm{N} 2+\mathrm{H} 2$

$\mathrm{NNH}+\mathrm{NH} 2=\mathrm{N} 2+\mathrm{NH} 3$

$\mathrm{NNH}+\mathrm{NH}=\mathrm{N} 2+\mathrm{NH} 2$

$\mathrm{NH} 2+\mathrm{NH}=\mathrm{N} 2 \mathrm{H} 2+\mathrm{H}$

$\mathrm{NH}+\mathrm{NH}=\mathrm{N} 2+\mathrm{H}+\mathrm{H}$

$\mathrm{NH} 2+\mathrm{N}=\mathrm{N} 2+\mathrm{H}+\mathrm{H}$

$\mathrm{N} 2 \mathrm{H} 2+\mathrm{M}=\mathrm{NNH}+\mathrm{H}+\mathrm{M}$

$\mathrm{N} 2 / 2 / \mathrm{H} 2 / 2 /$

$\mathrm{N} 2 \mathrm{H} 2+\mathrm{H}=\mathrm{NNH}+\mathrm{H} 2$

$\mathrm{N} 2 \mathrm{H} 2+\mathrm{NH}=\mathrm{NNH}+\mathrm{NH} 2$

$\mathrm{N} 2 \mathrm{H} 2+\mathrm{NH} 2=\mathrm{NH} 3+\mathrm{NNH}$

$\mathrm{NH} 2+\mathrm{NH} 2=\mathrm{N} 2 \mathrm{H} 2+\mathrm{H} 2$

$\mathrm{NH} 3+\mathrm{M}=\mathrm{NH} 2+\mathrm{H}+\mathrm{M}$

$\mathrm{N} 2 \mathrm{H} 3+\mathrm{H}=\mathrm{NH} 2+\mathrm{NH} 2$

$\mathrm{N} 2 \mathrm{H} 3+\mathrm{M}=\mathrm{N} 2 \mathrm{H} 2+\mathrm{H}+\mathrm{M}$

$\mathrm{N} 2 \mathrm{H} 3+\mathrm{NH}=\mathrm{NH} 2+\mathrm{N} 2 \mathrm{H} 2$

$\mathrm{NH} 2+\mathrm{NH} 2+\mathrm{M}=\mathrm{N} 2 \mathrm{H} 4+\mathrm{M}$

$\mathrm{H}+\mathrm{N} 2 \mathrm{H} 4=\mathrm{H} 2+\mathrm{N} 2 \mathrm{H} 3$

$\mathrm{NH} 2+\mathrm{N} 2 \mathrm{H} 4=\mathrm{NH} 3+\mathrm{N} 2 \mathrm{H} 3$

$\mathrm{NH}+\mathrm{H}+\mathrm{M}=\mathrm{NH} 2+\mathrm{M}$

$\mathrm{NH} 2+\mathrm{NH} 2=\mathrm{NH} 3+\mathrm{NH}$

$\mathrm{F}+\mathrm{NH} 3=\mathrm{NH} 2+\mathrm{HE}$

$\operatorname{SIF} 4=\operatorname{SIF} 3+\mathrm{F}$

$\mathrm{H}+\mathrm{SIF} 4=\mathrm{HE}+\mathrm{SIF} 3$

$\mathrm{NH} 2+\mathrm{SIF} 4=\mathrm{SIF} 3 \mathrm{NH} 2+\mathrm{F}$

$\mathrm{NH} 3+\mathrm{SIF} 3=\mathrm{SIF} 3 \mathrm{NH} 2+\mathrm{H}$

$\mathrm{NH} 3+\mathrm{SIF} 3=\mathrm{SIHF} 3+\mathrm{NH} 2$

END

\begin{tabular}{|c|c|c|c|c|c|}
\hline $0.100 E+19$ & -1.000 & 0.000 & & $D-L$ & \\
\hline $0.920 E+17$ & -0.600 & 0.000 & & & \\
\hline $0.300 E+14$ & 0.000 & 0.000 & $!$ & JAM & \\
\hline $0.100 E+15$ & 0.000 & 0.000 & $!$ & NH3 & CST \\
\hline $0.692 \mathrm{E}+14$ & 0.000 & 3650.000 & & & \\
\hline $0.636 \mathrm{E}+06$ & 2.390 & 10171.000 & ! & MICHA & IAEL \\
\hline $0.100 E+05$ & 0.000 & 0.000 & ! & JAM & \\
\hline $0.100 E+15$ & 0.000 & 0.000 & $!$ & JAM & \\
\hline $0.500 E+14$ & 0.000 & 0.000 & $!$ & JAM & \\
\hline $0.500 E+14$ & 0.000 & 0.000 & $!$ & JAM & \\
\hline $0.500 E+14$ & 0.000 & 0.000 & ! & $\mathrm{NH} 3 \mathrm{C}$ & EST \\
\hline $0.254 E+14$ & 0.000 & 0.000 & ! & NH3 & $\mathrm{CsT}$ \\
\hline $0.720 E+14$ & 0.000 & 0.000 & ! & $P G$ & \\
\hline $0.500 E+17$ & 0.000 & 50000.000 & ! & NH3 & $\mathrm{CST}$ \\
\hline $0.500 E+14$ & 0.000 & 1000.000 & $!$ & NH3 & CST \\
\hline $0.100 E+14$ & 0.000 & 1000.000 & ! & NH3 & CST \\
\hline $0.100 E+14$ & 0.000 & 1000.000 & $!$ & NH3 & $\operatorname{CST}$ \\
\hline $0.500 E+12$ & 0.000 & 0.000 & ! & NH3 & CST \\
\hline $0.140 E+17$ & 0.000 & 90600.000 & ! & MSGK & \\
\hline 1. $60 \mathrm{E}+12$ & 0.0 & 0.0 & ! & MSGK & \\
\hline $3.50 E+16$ & 0.0 & 46000.0 & ! & MSGK & \\
\hline $2.00 E+13$ & 0.0 & 0.0 & ! & MSCK & \\
\hline $3.00 \mathrm{E}+20$ & -1.0 & 0.0 & ! & MSGK & \\
\hline 1. $30 E+13$ & 0.0 & 2500.0 & ! & MSGK & \\
\hline $3.90 E+12$ & 0.0 & 1500.0 & ! & MSGK & \\
\hline $2.00 E+16$ & -0.5 & 0.0 & ! & MSGK & \\
\hline $5.00 \mathrm{E}+12$ & 0.0 & 10000.0 & ! & MSGK & \\
\hline $4.27 E+11$ & 0.5 & 800.0 & ! & KOND & RATIEV \\
\hline $3.00 E+12$ & 0.0 & 147170.0 & ! & PHO\& & $\mathrm{MEC}$ \\
\hline $1.00 E+13$ & 0.0 & 50000.0 & ! & PHO\& & $M E C$ \\
\hline $1.00 E+11$ & 0.0 & 40950.0 & 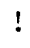 & GUES & \\
\hline $1.00 E+11$ & 0.0 & 5000.0 & ! & GUES & \\
\hline $1.00 \mathrm{E}+11$ & 0.0 & 10000.0 & ! & PHO\& & MEC \\
\hline
\end{tabular}


Output from CHEMKIN Interpreter for the Example

CHEMKIN INTERPRETER OUTPUT: CHEMKIN-II Version 2.0 Aug. 1990 DOUBLE PRECISION

\begin{tabular}{ll} 
ELEMENTS & ATOMIC \\
CONSIDERED & WEIGHT \\
\hline 1. H & 1.00797 \\
2. N & 14.0067 \\
3. SI & 28.0860 \\
4. F & 18.9984 \\
\hline
\end{tabular}

\begin{tabular}{|c|c|c|c|c|c|c|c|c|c|}
\hline \multirow{6}{*}{$\begin{array}{l}\text { SPECIES } \\
\text { CONSIDERED }\end{array}$} & \multirow{6}{*}{$\begin{array}{l}\mathrm{P} \\
\mathrm{H} \\
\mathrm{A} \\
\mathrm{S} \\
\mathrm{E}\end{array}$} & \multicolumn{8}{|c|}{ C } \\
\hline & & \multicolumn{8}{|c|}{$\mathrm{H}$} \\
\hline & & \multicolumn{8}{|l|}{ A } \\
\hline & & \multicolumn{8}{|l|}{$\mathrm{R}$} \\
\hline & & G & MOLECULAR & \multicolumn{2}{|c|}{ TEMPERATURE } & \multicolumn{4}{|c|}{ ELEMENT COUNT } \\
\hline & & $E$ & WEIGHT & LOW & HIGH & $\mathrm{H}$ & $\mathrm{N}$ & SI & $E$ \\
\hline 1. $\mathrm{H} 2$ & G & 0 & 2.01594 & 300.0 & 5000.0 & 2 & 0 & 0 & 0 \\
\hline 2. $\mathrm{H}$ & G & 0 & 1.00797 & 300.0 & 5000.0 & 1 & 0 & 0 & 0 \\
\hline 3. N2 & G & 0 & 28.01340 & 300.0 & 5000.0 & 0 & 2 & c & 0 \\
\hline 4. $N$ & G & 0 & 14.00670 & 300.0 & 5000.0 & 0 & 1 & 0 & 0 \\
\hline 5. $\mathrm{NH}$ & G & 0 & 15.01467 & 300.0 & 5000.0 & 1 & 1 & 0 & 0 \\
\hline 6. $\mathrm{NH} 2$ & G & 0 & 16.02264 & 300.0 & 5000.0 & 2 & 1 & 0 & 0 \\
\hline 7. $\mathrm{NNH}$ & G & 0 & 29.02137 & 250.0 & 4000.0 & 1 & 2 & 0 & 0 \\
\hline 8. $\mathrm{N} 2 \mathrm{H} 2$ & G & 0 & 30.02934 & 300.0 & 5000.0 & 2 & 2 & 0 & 0 \\
\hline 9. $\mathrm{N} 2 \mathrm{H} 3$ & G & 0 & 31.03731 & 300.0 & 5000.0 & 3 & 2 & 0 & 0 \\
\hline 10. $\mathrm{N} 2 \mathrm{H} 4$ & G & 0 & 32.04528 & 300.0 & 5000.0 & 4 & 2 & 0 & 0 \\
\hline 11. $\mathrm{HF}$ & G & 0 & 20.00637 & 300.0 & 5000.0 & 1 & 0 & 0 & 1 \\
\hline 12. $E$ & G & 0 & 18.99840 & 300.0 & 5000.0 & 0 & 0 & 0 & 1 \\
\hline 13. SIF4 & G & 0 & 104.07960 & 300.0 & 5000.0 & 0 & 0 & 1 & 4 \\
\hline 14. SIF 3 & G & 0 & 85.08120 & 300.0 & 3000.0 & 0 & 0 & 1 & 3 \\
\hline 15. SIHF 3 & G & 0 & 86.08917 & 300.0 & 3000.0 & 1 & 0 & 1 & 3 \\
\hline 16. SIF $3 \mathrm{NH} 2$ & G & 0 & 101.10384 & 300.0 & 3000.0 & 2 & 1 & 1 & 3 \\
\hline 17. NH3 & $G$ & 0 & 17.03061 & 300.0 & 5000.0 & 3 & 1 & 0 & 0 \\
\hline
\end{tabular}




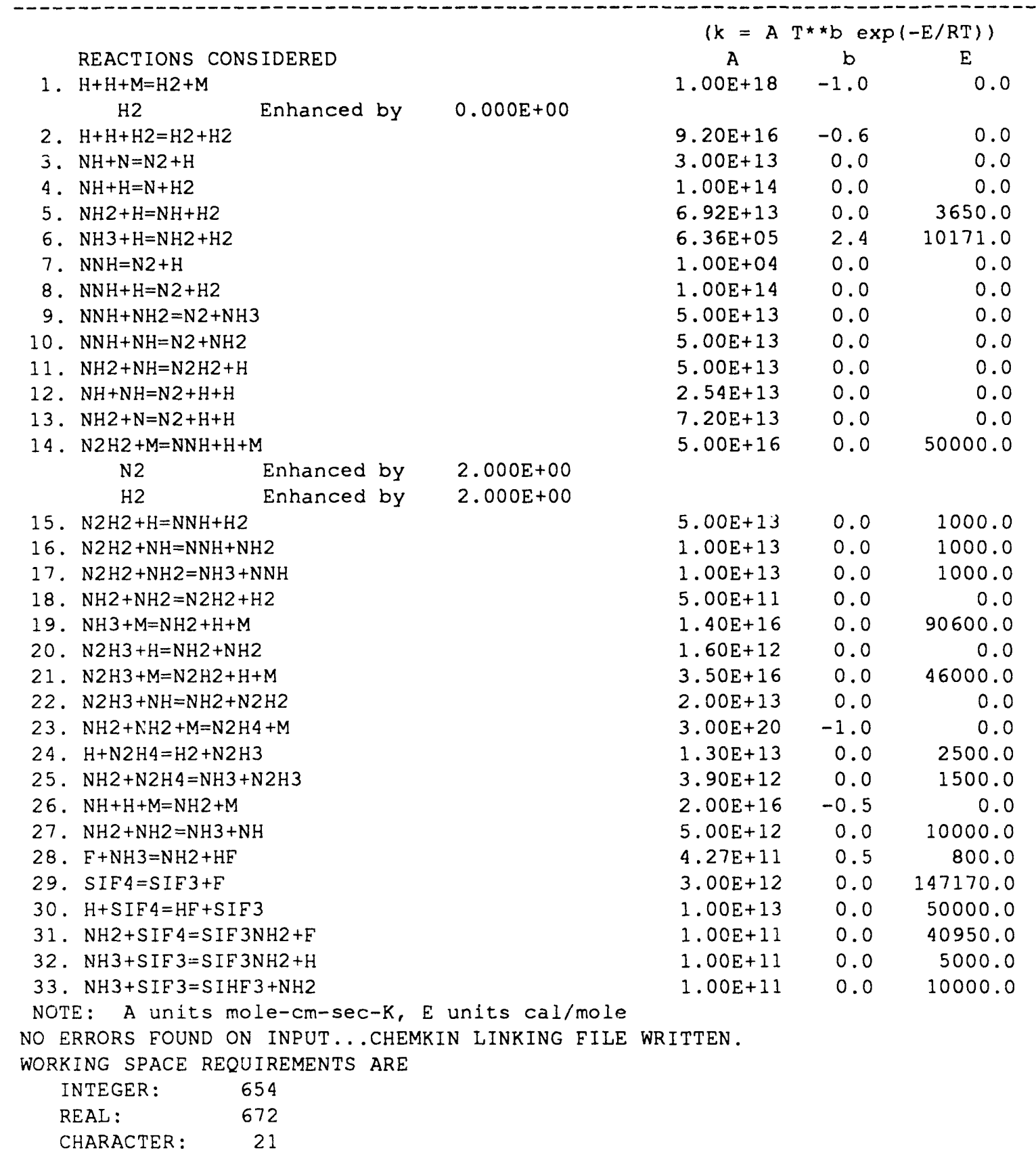




\section{Input to SURFACE CHEMKIN Interpreter for the Example}

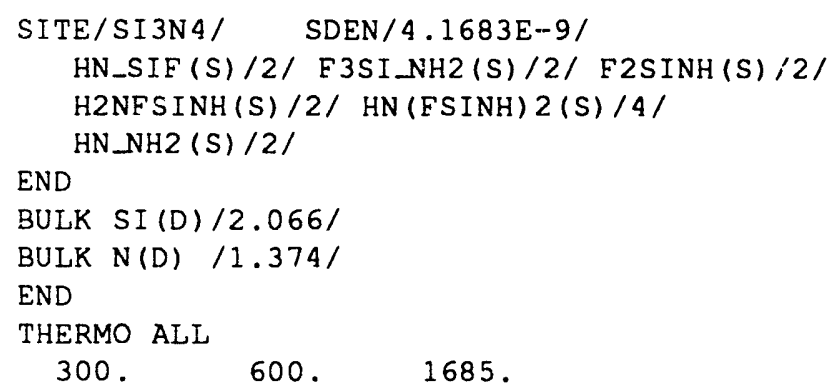

HN_SIF(S) J $3 / 67 \mathrm{~N} \quad 1 \mathrm{H} \quad 1 \mathrm{SI} \quad 1 \mathrm{~F} \quad 1 \mathrm{~S} \quad 300.000 \quad 1685.000 \quad 1$

$\begin{array}{llllllll}0.24753989 \mathrm{E} & 01 & 0.88112187 \mathrm{E}-03-0.20939481 \mathrm{E}-06 & 0.42757187 \mathrm{E}-11 & 0.16006564 \mathrm{E}-13 & 2\end{array}$

$\begin{array}{lllllllll}-0.81255620 \mathrm{E} & 03-0.12188747 \mathrm{E} & 02 & 0.84197538 \mathrm{E} & 00 & 0.33710416 \mathrm{E}-02-0.13077030 \mathrm{E}-04 & 3\end{array}$

$0.97593603 \mathrm{E}-08-0.27279380 \mathrm{E}-11-0.52486288 \mathrm{E} \quad 03-0.452 ? 2678 \mathrm{E} 01 \quad 4$

HN_NH2(S) J $3 / 67 \mathrm{~N} \quad 2 \mathrm{H} \quad 3 \mathrm{SI}$ OF OS $300.000 \quad 1685.000$

$0.24753989 \mathrm{E} \quad 01 \quad 0.88112187 \mathrm{E}-03-0.20939481 \mathrm{E}-06 \quad 0.42757187 \mathrm{E}-11 \quad 0.16006564 \mathrm{E}-13$

$-0.81255620 \mathrm{E} \quad 03-0.12188747 \mathrm{E} \quad 02 \quad 0.84197538 \mathrm{E}$ तO $0.83710416 \mathrm{E}-02-0.13077030 \mathrm{E}-04$

$0.97593603 \mathrm{E}-08-0.27279380 \mathrm{E}-11-0.52486288 \mathrm{E} \quad 03-0.45272678 \mathrm{E} \quad 01$

F3SI_NH2 (S) $\quad \mathrm{J} 3 / 67 \mathrm{~N} \quad 1 \mathrm{H} \quad 2 \mathrm{SI} \quad 1 \mathrm{~F} \quad 3 \mathrm{~S} \quad 300.000 \quad 1685.000$

$0.24753989 \mathrm{E} \quad 01 \quad 0.88112187 \mathrm{E}-03-0.20939481 \mathrm{E}-06 \quad 0.42757187 \mathrm{E}-11 \quad 0.16006564 \mathrm{E}-13$

$\begin{array}{lllllll}-0.81255620 \mathrm{E} & 03-0.12188747 \mathrm{E} & 02 & 0.84197538 \mathrm{E} & 00 & 0.83710416 \mathrm{E}-02-0.13077030 \mathrm{E}-04 & 3\end{array}$

$0.97593603 \mathrm{E}-08-0.27279380 \mathrm{E}-11-0.52486288 \mathrm{E} \quad 03-0.45272678 \mathrm{E} 01$

F2SINH(S) J $3 / 67 \mathrm{~N} \quad 1 \mathrm{H} \quad 1 \mathrm{SI} \quad 1 \mathrm{~F} \quad 2 \mathrm{~S} \quad 300.000 \quad 1685.000$

$0.24753989 \mathrm{E} \quad 01 \quad 0.88112187 \mathrm{E}-03-0.20939481 \mathrm{E}-06 \quad 0.42757187 \mathrm{E}-11 \quad 0.16006564 \mathrm{E}-13$

$\begin{array}{llllll}-0.81255620 \mathrm{E} & 03-0.12188747 \mathrm{E} & 02 & 0.84197538 \mathrm{E} & 00 & 0.83710416 \mathrm{E}-02-0.13077030 \mathrm{E}-04\end{array}$

$0.97593603 E-08-0.27279380 E-11-0.52486288 \mathrm{E} \quad 03-0.45272678 \mathrm{E} 01$

H2NESINH(S) J $3 / 67 \mathrm{~N} \quad 2 \mathrm{H} \quad 3 \mathrm{SI} \quad 1 \mathrm{~F} \quad$ IS $300.000 \quad 1685.000$

$\begin{array}{lllllll}0.24753989 \mathrm{E} & 01 & 0.88112187 \mathrm{E}-03-0.20939481 \mathrm{E}-06 & 0.42757187 \mathrm{E}-11 & 0.16006564 \mathrm{E}-13\end{array}$

$-0.81255620 \mathrm{E} \quad 03-0.12188747 \mathrm{E} \quad 02 \quad 0.84197538 \mathrm{E} \quad 00 \quad 0.83710416 \mathrm{E}-02-0.13077030 \mathrm{E}-04$

$0.97593603 E-08-0.27279380 E-11-0.52486288 E \quad 03-0.45272678 E \quad 01$

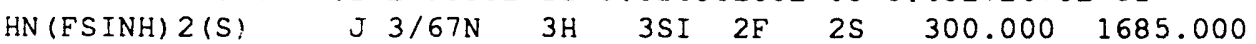

$\begin{array}{llllllll}0.24753989 E & 01 & 0.88112187 E-03-0.20939481 E-06 & 0.42757187 E-11 & 0.16006564 E-13\end{array}$

$-0.81255620 \mathrm{E} \quad 03-0.12188747 \mathrm{E} \quad 02 \quad 0.84197538 \mathrm{E} \quad 00 \quad 0.83710416 \mathrm{E}-02-0.13077030 \mathrm{E}-04$

$0.97593603 \mathrm{E}-08-0.27279380 \mathrm{E}-11-0.52486288 \mathrm{E} \quad 03-0.45272678 \mathrm{E} \quad 01$

SI (D) J $3 / 67 S I \quad 100 \quad 000 \quad 000$ OS $300.000 \quad 1685.000$

$\begin{array}{lllllll}0.24753989 \mathrm{E} & 01 & 0.88112187 \mathrm{E}-03-0.20939481 \mathrm{E}-06 & 0.42757187 \mathrm{E}-11 & 0.16006564 \mathrm{E}-13\end{array}$

$-0.81255620 \mathrm{E} \quad 03-0.12188747 \mathrm{E} \quad 02 \quad 0.84197538 \mathrm{E} \quad 00 \quad 0.83710416 \mathrm{E}-02-0.13077030 \mathrm{E}-04$

$0.97593603 E-08-0.27279380 E-11-0.52486288 E \quad 03-0.45272678 E \quad 01$

$N(D) \quad J 3 / 67 \mathrm{~N} \quad 100 \quad 000 \quad 000$ OS $300.000 \quad 1685.000$

$\begin{array}{lllllll}0.24753989 E & 01 & 0.88112187 E-03-0.20939481 E-06 & 0.42757187 E-11 & 0.16006564 E-13\end{array}$

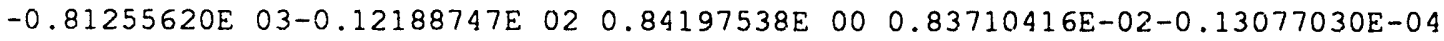
$0.97593603 \mathrm{E}-08-0.27279380 \mathrm{E}-11-0.52486288 \mathrm{E} \quad 03-0.45272678 \mathrm{E} \quad 01$

END

REACTIONS

\begin{tabular}{|c|c|c|c|c|c|c|c|}
\hline NH3 & + & HN_SIF (S) & $\Rightarrow$ & $\mathrm{HN} \_\mathrm{NH} 2(\mathrm{~S})$ & $+S I(D)$ & $+\mathrm{HF}$ & $7.562 \mathrm{E} 08$ \\
\hline SIF4 & + & HN_NH2 (S) & $\Rightarrow$ & F3SI_NH2 (S) & $+N(D)$ & $+\mathrm{HF}$ & $3.0967 \mathrm{E} 8$ \\
\hline F 3SI_NH2 (S) & & & $\Rightarrow$ & F2SINH $(S)$ & & $+\mathrm{HF}$ & $\begin{array}{lll}1.0 E 05 & 0.0 & 0.0\end{array}$ \\
\hline NH3 & + & F2SINH (S) & $\Rightarrow$ & H2NFS INH (S) & & $+\mathrm{HF}$ & $\begin{array}{lll}7.562 E 08 & 0.5 & 0.0\end{array}$ \\
\hline H2NFSINH (S) & + & F2SINH (S) & $\Rightarrow$ & HN (ESINH) 2 (S & & $+H F$ & $\begin{array}{lll}1.0 E 15 & 0.0 & 0.0\end{array}$ \\
\hline HN (FSINH) 2 (S) & + & F2SINH $(S)$ & $\cdots$ & $3 \mathrm{HN} \_S I F(S)$ & $+N(D)$ & $+\mathrm{HF}$ & 0.00 .0 \\
\hline
\end{tabular}




\section{Output from SURFACE CHEMKIN Interpreter for the Example}

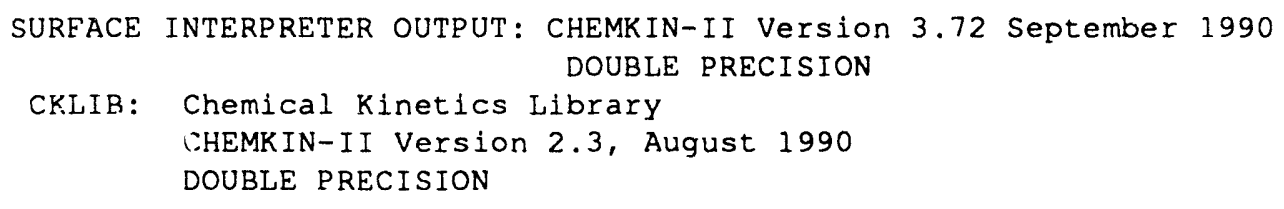

$\begin{array}{llll}\text { MOLECULAR } & & \text { ELEMENT COUNT } \\ \text { CONSIDERED } & \text { WEIGHT } & \text { Nensity SI F }\end{array}$

Gas phase species:

1. $\mathrm{H} 2$

2. $\mathrm{H}$

3. $\mathrm{N} 2$

4. N

5. $\mathrm{NH}$

6. NH2

7. $\mathrm{NNH}$

8. $\mathrm{N} 2 \mathrm{H} 2$

9. N2H3

10. $\mathrm{N} 2 \mathrm{H} 4$

11. $\mathrm{HF}$

12. $\mathrm{F}$

13. SIF4

14. $\operatorname{SIF} 3$

15. SIHF 3

16. SIF $3 \mathrm{NH} 2$

17. $\mathrm{NH} 3$

SITE: SI3N4

18. HN_SIF (S)

19. F3SI_NH2 (S)

20. F2SINH(S)

21. H2NESINH (S)

22. $\mathrm{HN}(\mathrm{FSINH}) 2(\mathrm{~S})$

23. HN_NH2 (S)

BULK: BULKI

24. $S I(D)$

BULK: BULK2

25. $N(D)$

2.01594
1.00797
28.01340
14.00670
15.01467
16.02264
29.02137
30.02934
31.03731
32.04528
20.00637
18.99840
104.07960
85.08120
86.08917
101.10384
17.03061
$\quad$

62.09907

101.10384

81.09747

78.12171

139.21281

31.03731

$28.08600 \quad 0.207 \mathrm{E}+01 \mathrm{~g} / \mathrm{cm}^{\star \star} 3$

$\begin{array}{lllll}2 & 1 & 1 & 1 & 1\end{array}$

$\begin{array}{lllll}2 & 2 & 1 & 1 & 3\end{array}$

$\begin{array}{lllll}2 & 1 & 1 & 1 & 2\end{array}$

$\begin{array}{lllll}2 & 3 & 2 & 1 & 1\end{array}$

$\begin{array}{lllll}4 & 3 & 3 & 2 & 2\end{array}$

$\begin{array}{lllll}2 & 3 & 2 & 0 & 0\end{array}$

$14.00670 \quad 0.137 \mathrm{E}+01 \quad \mathrm{~g} / \mathrm{cm}^{\star} \star 3 \quad 001000$

\begin{tabular}{|c|c|c|c|}
\hline \multicolumn{4}{|c|}{$\left(k=A T^{\star \star} b \exp (-E / R T)\right)$} \\
\hline SURFACE REACTIONS CONSIDERED & A & b & E \\
\hline 1. $\mathrm{NH} 3+\mathrm{HN} \_\mathrm{SIF}(\mathrm{S}) \Rightarrow \mathrm{HN} \_\mathrm{NH} 2(\mathrm{~S})+\mathrm{SI}(\mathrm{D})+\mathrm{HF}$ & $7.56 \mathrm{E}+08$ & 0.5 & 0.0 \\
\hline 2. $\mathrm{SIF}_{4}+\mathrm{HN} \_\mathrm{NH} 2(\mathrm{~S}) \Rightarrow \mathrm{E} 3 \mathrm{SI} \mathrm{N}_{\mathrm{N}} 2(\mathrm{~S})+\mathrm{N}(\mathrm{D})+\mathrm{HF}$ & $3.10 E+08$ & 0.5 & 0.0 \\
\hline 3. F3SI_NH2 $(S)=>$ F 2 SINH $(S)+H F$ & $1.00 \mathrm{E}+05$ & 0.0 & 0.0 \\
\hline 4. NH3+F2SINH $(S)=>$ H2NFSINH $(S)+H F$ & $7.56 E+08$ & 0.5 & 0.0 \\
\hline 5. $H 2 N F S I N H(S)+F 2 \operatorname{SINH}(S) \Rightarrow+H N(F S I N H) 2(S)+H F$ & $1.00 E+15$ & 0.0 & 0.0 \\
\hline 6. HN (FSINH) $2(S)+F 2 S I N H(S)$ & $1.00 E+15$ & 0.0 & 0.0 \\
\hline
\end{tabular}
$\Rightarrow 3$ HN_SIF $(S)+\mathrm{N}(D)+\mathrm{HF}$

NOTE: A units mole, E units cal/mole

NO ERRORS FOUND ON INPUT...SURFACE LINKING EILE WRITTEN.

WORKING SPACE REQUIREMENTS ARE

$\begin{array}{lr}\text { INTEGER: } & 398 \\ \text { REAL: } & 576 \\ \text { CHARACTER : } & 33\end{array}$


$\underline{\text { Input to SPIN for the Example }}$

\begin{tabular}{|c|c|c|}
\hline ENRG & & \\
\hline OMEG & 1000 & \\
\hline TDSK & 1713 & \\
\hline TINF & 300 & \\
\hline NPTS & 11 & \\
\hline XEND & 10.0 & \\
\hline PRES & $2.3684 E-3$ & \\
\hline GRAD & .1 & \\
\hline CURV & .5 & \\
\hline REAC & SIF 4 & 0.14286 \\
\hline REAC & NH3 & 0.85714 \\
\hline PROD & SIF 4 & 0.123 \\
\hline PROD & NH3 & 0.8547 \\
\hline PROD & $\mathrm{HE}$ & 0.02231 \\
\hline SURE & HN_SIF (S) & $6.2511 \mathrm{E}-02$ \\
\hline SURE & HN_NH2 (S) & 0.915873 \\
\hline SURF & F 3SI_NH2 (S) & $2.3540 E-04$ \\
\hline SURE & F2SINH (S) & $2.0837 E-02$ \\
\hline SURE & H2NFS INH (S) & $1.8063 E-04$ \\
\hline SURF & $\mathrm{HN}$ (ESINH) 2 (S) & $3.6127 E-04$ \\
\hline $\mathrm{ACT}$ & $S I(D)$ & 1.0 \\
\hline $\mathrm{ACT}$ & $N(D)$ & 1.0 \\
\hline ATOL & $1 \cdot E-8$ & \\
\hline RTOL & 1.E-5 & \\
\hline ATIM & 1.E-8 & \\
\hline RTIM & 1.E-3 & \\
\hline PRNT & 1 & \\
\hline NADP & 4 & \\
\hline$X C E N$ & .4 & \\
\hline WMIX & .35 & \\
\hline TIME & $100 \quad 1.0 \mathrm{E}-8$ & \\
\hline TIM2 & $100 \quad 1.0 \mathrm{E}-8$ & \\
\hline SFLR & $-1.0 E-3$ & \\
\hline TEMP & $0 . \quad 1713$. & \\
\hline TEMP & 1713. & \\
\hline TEMP & 300 & \\
\hline END & & \\
\hline
\end{tabular}



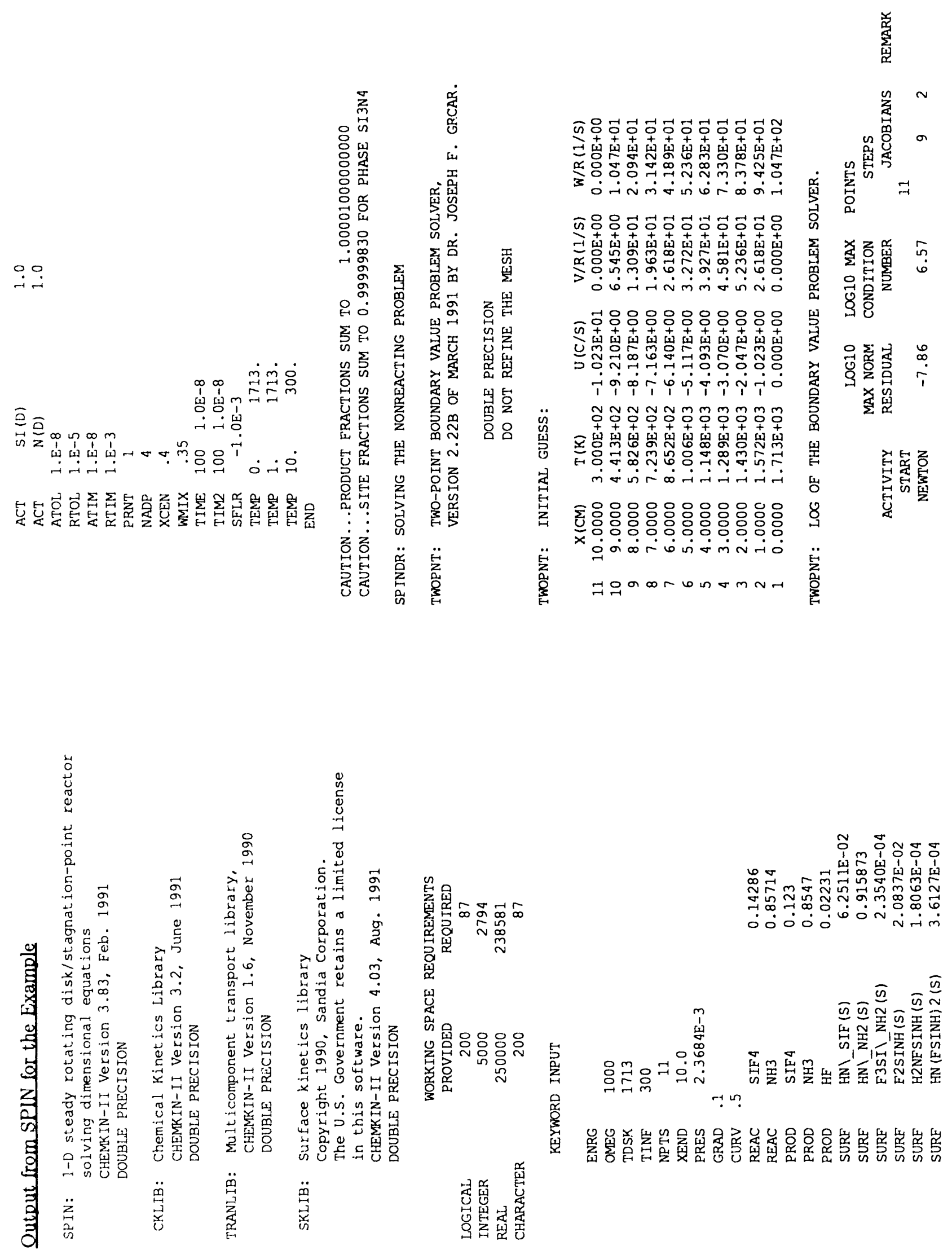


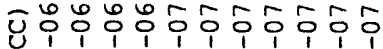

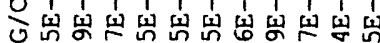

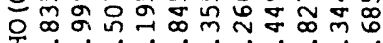

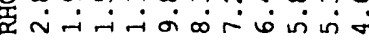

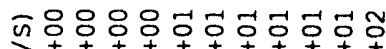

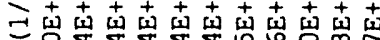

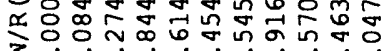

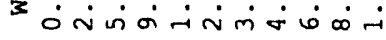

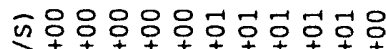

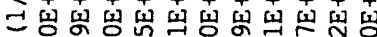

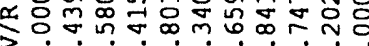

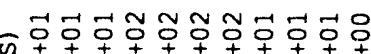

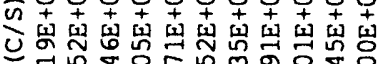

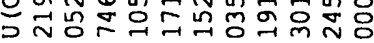
फक्षिनंभक्षिक

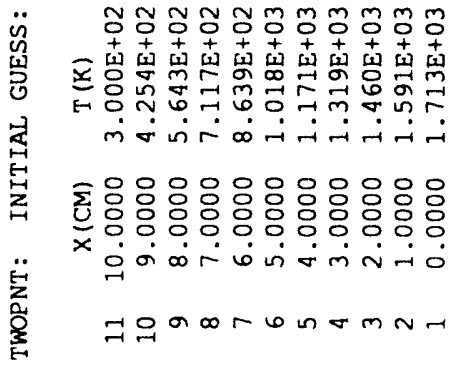

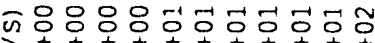

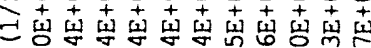

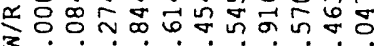
.

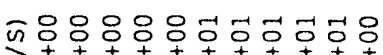

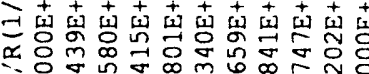

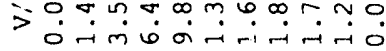

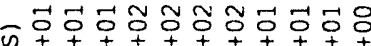

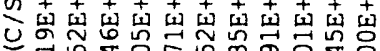

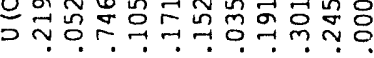
१क्षिसतसक्षिक

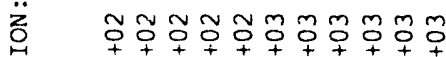

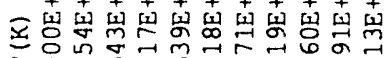

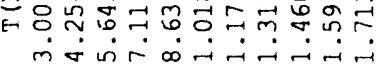

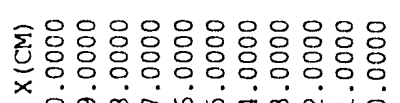

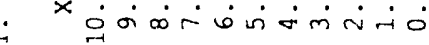

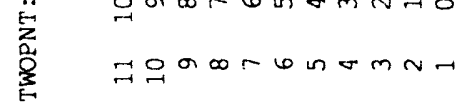

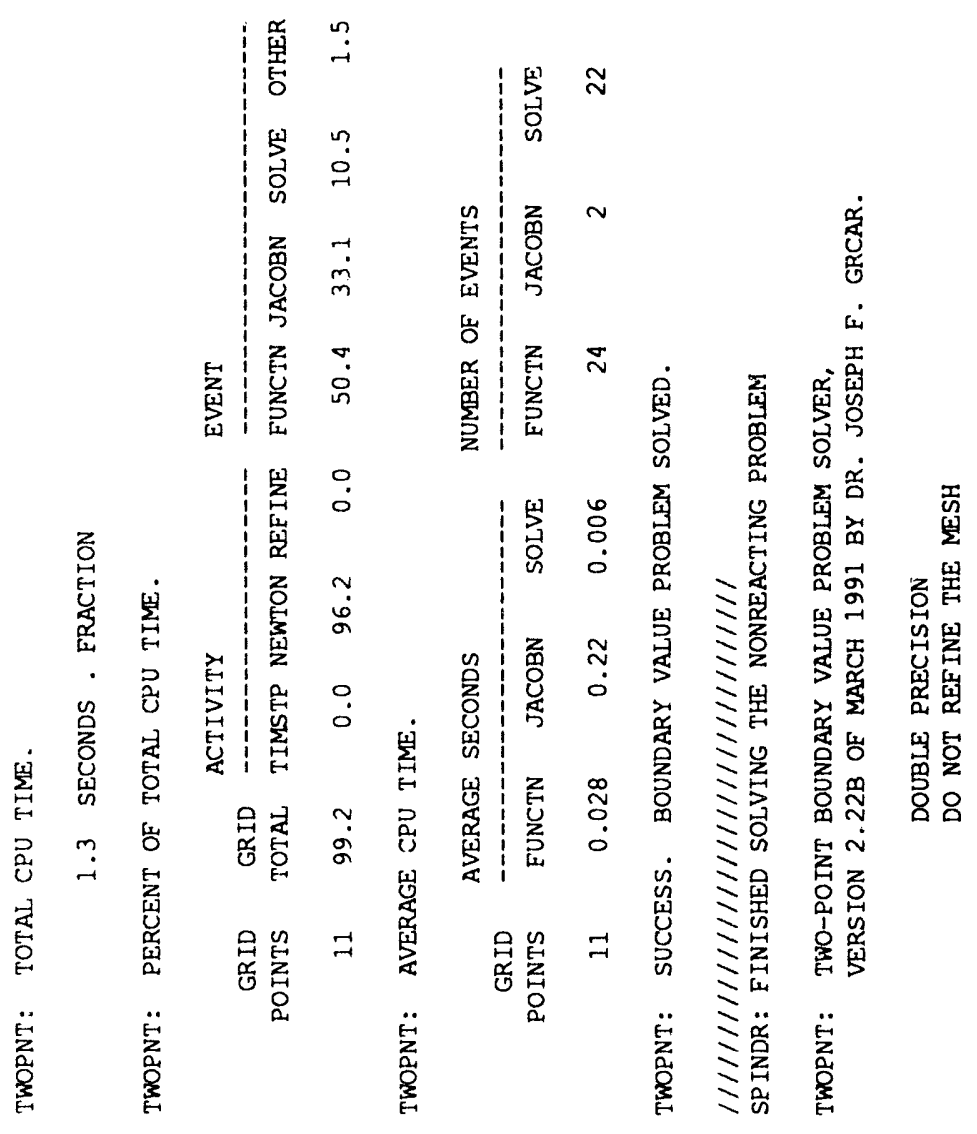


웅ㅇㅇㅇㅇㅇ웅ㅇㅇ웅웅우

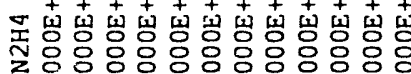
0.

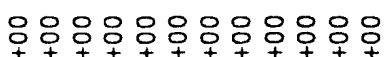

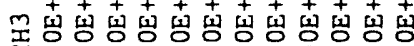

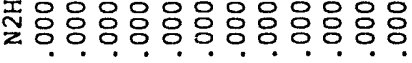
ல் $0 \dot{0} 0 \dot{0} 0 \dot{0} 0 \dot{0}$

웅웅ㅇㅇㅇㅇㅇㅇㅇㅇㅇㅇㅇㅇㅜ

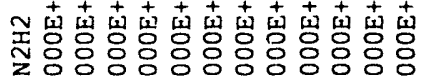

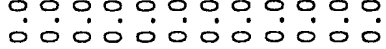

응ㅇㅇㅇㅇㅇㅇㅇㅇㅇㅇㅇㅇㅇㅇㅇㅇㅁ

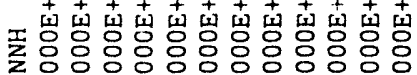
○ं0் $0 \dot{0} \dot{0} \dot{0} \dot{0}$

응ㅇㅇㅇㅇㅇㅇㅇㅇㅇㅇㅇㅇㅇㅇㅇㅇㅇㅇㅇㅇ

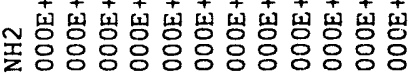
ó0ं0் 0.

웅우웅우웅우웅우웅우우

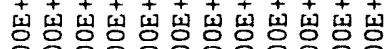

득ㅇㅇㅇㅇㅇㅇㅇㅇㅇㅇㅇㅇㅇㅇㅇㅇㅇㅇㅇㅇ

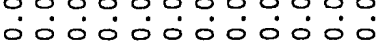

88:88:88:88:

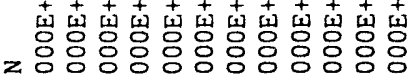

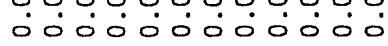

응ㅇㅇㅇㅇㅇㅇㅇㅇㅇㅇㅇㅇㅇㅇㅇㅇㅇㅇㅇ

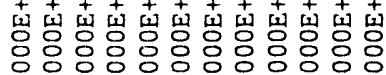

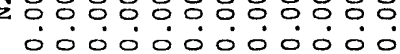

웅웅ㅇㅇ웅웅ㅇㅇ웅ㅇㅇㅇㅇㅇ

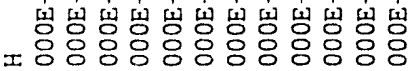
○ं0ं $0 \dot{0} 0 \dot{0} 0 \dot{0} 0$

응ㅇㅇㅇㅇㅇㅇㅇㅇㅇㅇㅇㅇㅇㅇㅇㅇㅇㅇ

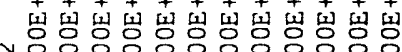

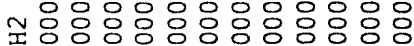
○ं $\dot{0} \dot{0} \dot{0} \dot{0} 0 \dot{0} \dot{0}$

능ㅇㅇㅇㅇㅇㅇㅇㅇㅇㅇㅇㅇㅇㅇㅇㅇㅇㅛ

х

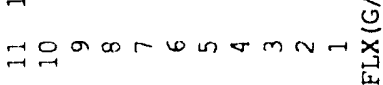

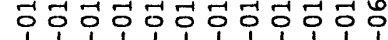

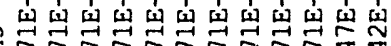

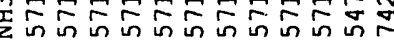
$\infty \infty \infty \infty \infty \infty \infty \infty \infty \infty N$

꽁ㅇㅇㅇㅇㅇㅇㅇㅇㅇㅇㅇㅇㅇㅇㅇㅇㅇㅇㅇㅇㅇㅇ 至 성ㅇㅇㅇㅇㅇㅇㅇㅇㅇㅇㅇㅇㅇㅇㅇㅇㅇㅇㅇㅇㅇㅇㅇ ó 웅ㅇㅇㅇㅇㅇㅇㅇㅇㅇㅇㅇㅇㅇㅇㅇㅇㅇㅇㅇㅇ

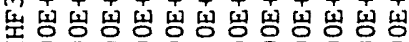
4 லं0் $0 \dot{0} \dot{0} 0 \dot{0} 0 \dot{0}$

응ㅇㅇㅇㅇㅇㅇㅇㅇㅇㅇㅇㅇㅇㅇㅇ

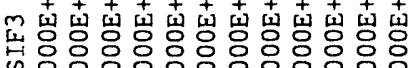

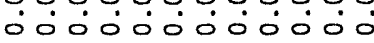

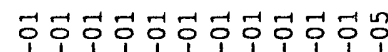

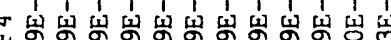

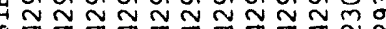

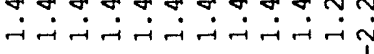

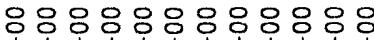

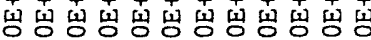

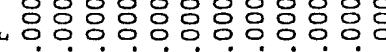

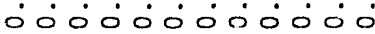

응ㅇㅇㅇㅇㅇㅇㅇㅇㅇㅇㅇㅇㅇㅁㄷㄴㅇ

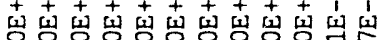

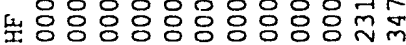
ó00் 0 0ं0்

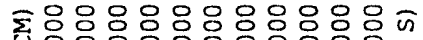

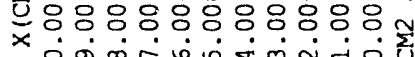

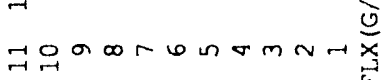

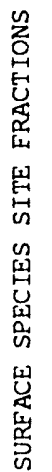

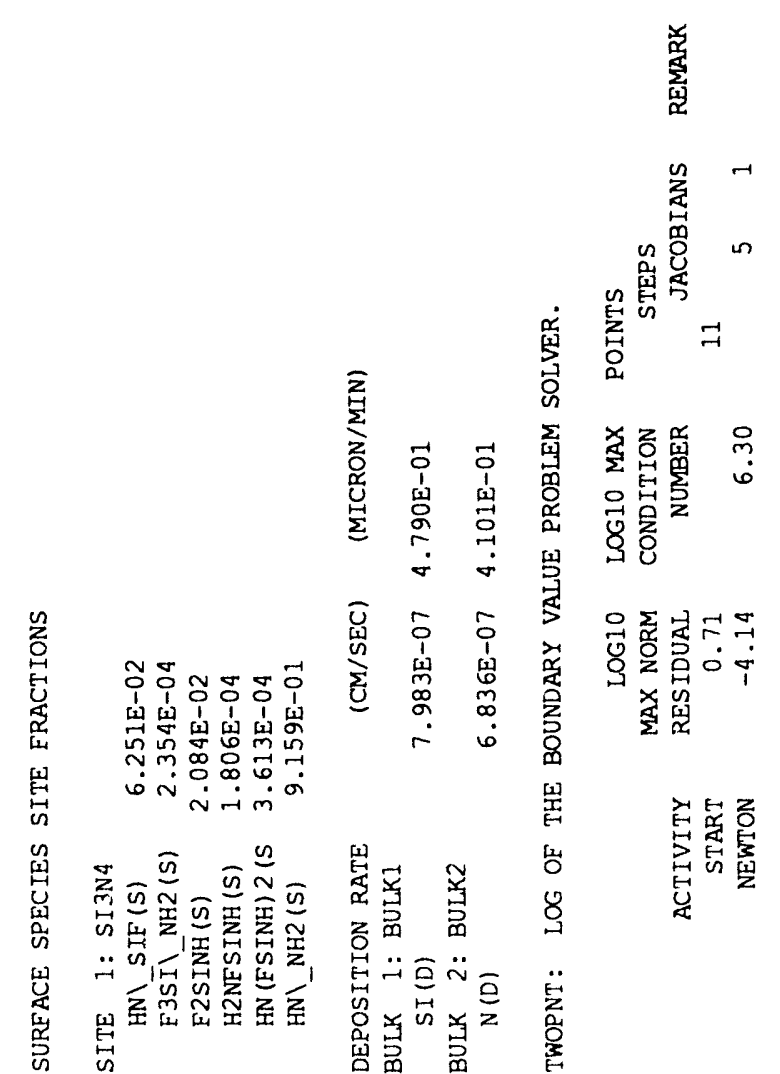




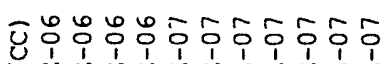

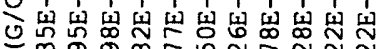
о

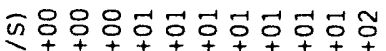

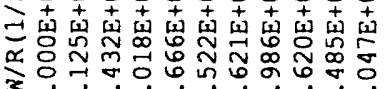

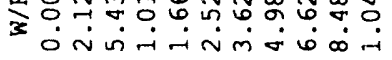

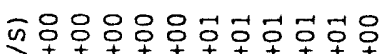

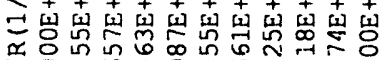
>

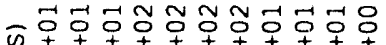

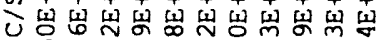

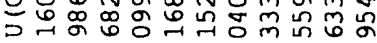

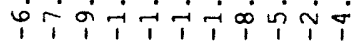

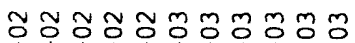

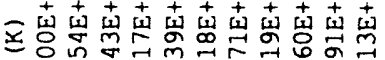

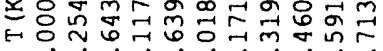

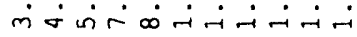

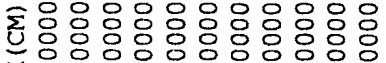
×

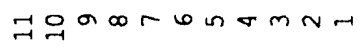

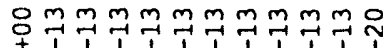

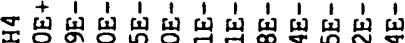

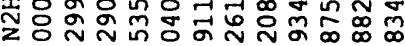

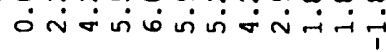

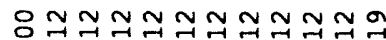

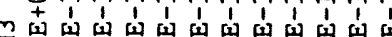

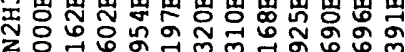
$\dot{0}-\dot{-i} \dot{\sim} \dot{v} \dot{v}-\dot{-} \dot{-}$

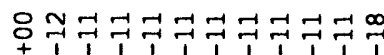

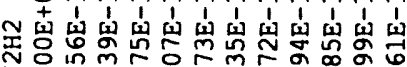

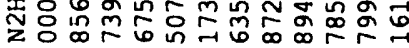
or $\dot{0} \dot{\sim} \dot{\sigma} \dot{\sigma} \dot{\sigma} \dot{\sigma} \dot{\sigma} \dot{\sigma}$

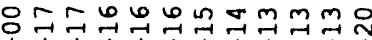

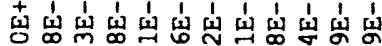

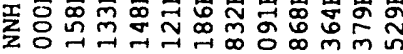

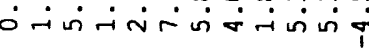

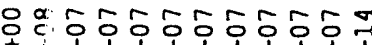

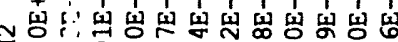

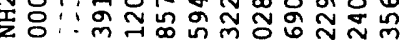

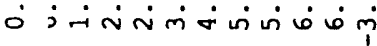

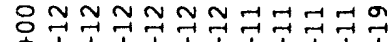

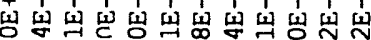

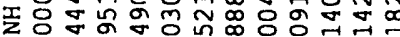

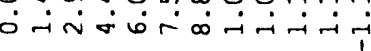
웅

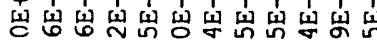

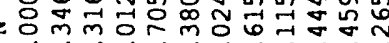
$\dot{0} \dot{0} \dot{\sim} \dot{\sim} \dot{m} \dot{\sigma} \dot{\sigma}$ in $\sin$

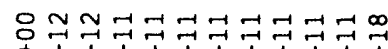

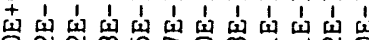
중중 வ $\dot{0} \dot{-} \dot{\sim} \dot{\sim} \dot{m} \dot{m} \dot{m}$

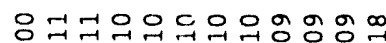

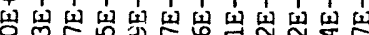

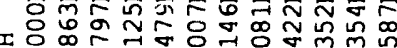

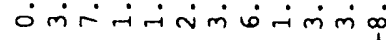
ㅇㅇㅇㅇㅇㅇㅇㅇㅇㅇㅇㅇㅇㅠ 屯'

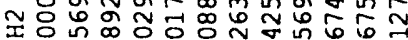

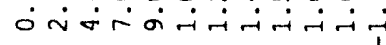

칭용ㅇㅇㅇㅇㅇㅇㅇㅇㅇㅇㅇㅇㅇㅇㅇㅇㅇㅇㅇㅇ 증ㅇㅇㅇㅇㅇㅇㅇㅇㅇㅇㅇㅇㅇㅇㅇㅇㅇㅇㅇㅇㄴㄷㄴ 엉aㅇ

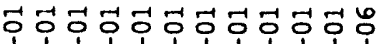

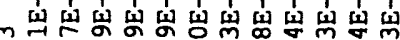

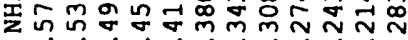
$\infty \dot{\infty} \infty \dot{\infty} \dot{\infty} \dot{\infty} \infty \dot{\infty} \infty \dot{\infty}$

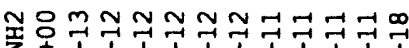

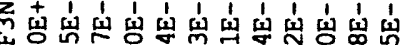

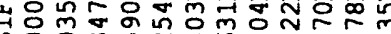
0

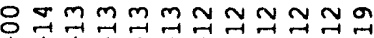

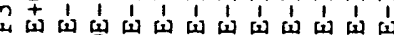

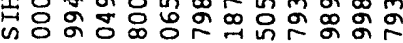

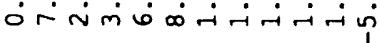
음

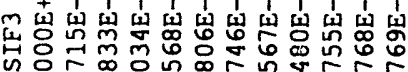

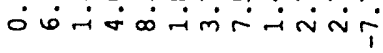

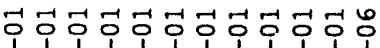

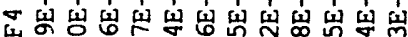

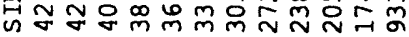

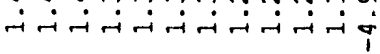
음

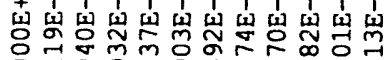

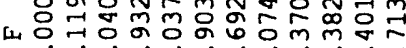

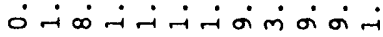

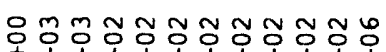

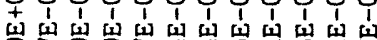

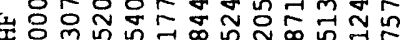

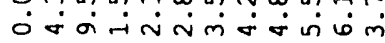

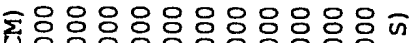

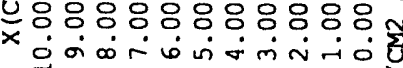

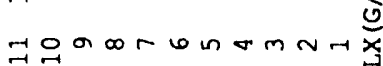

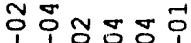

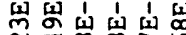

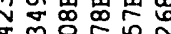
ni

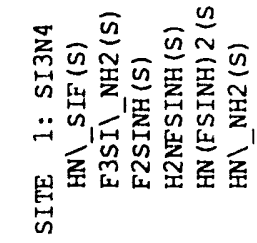




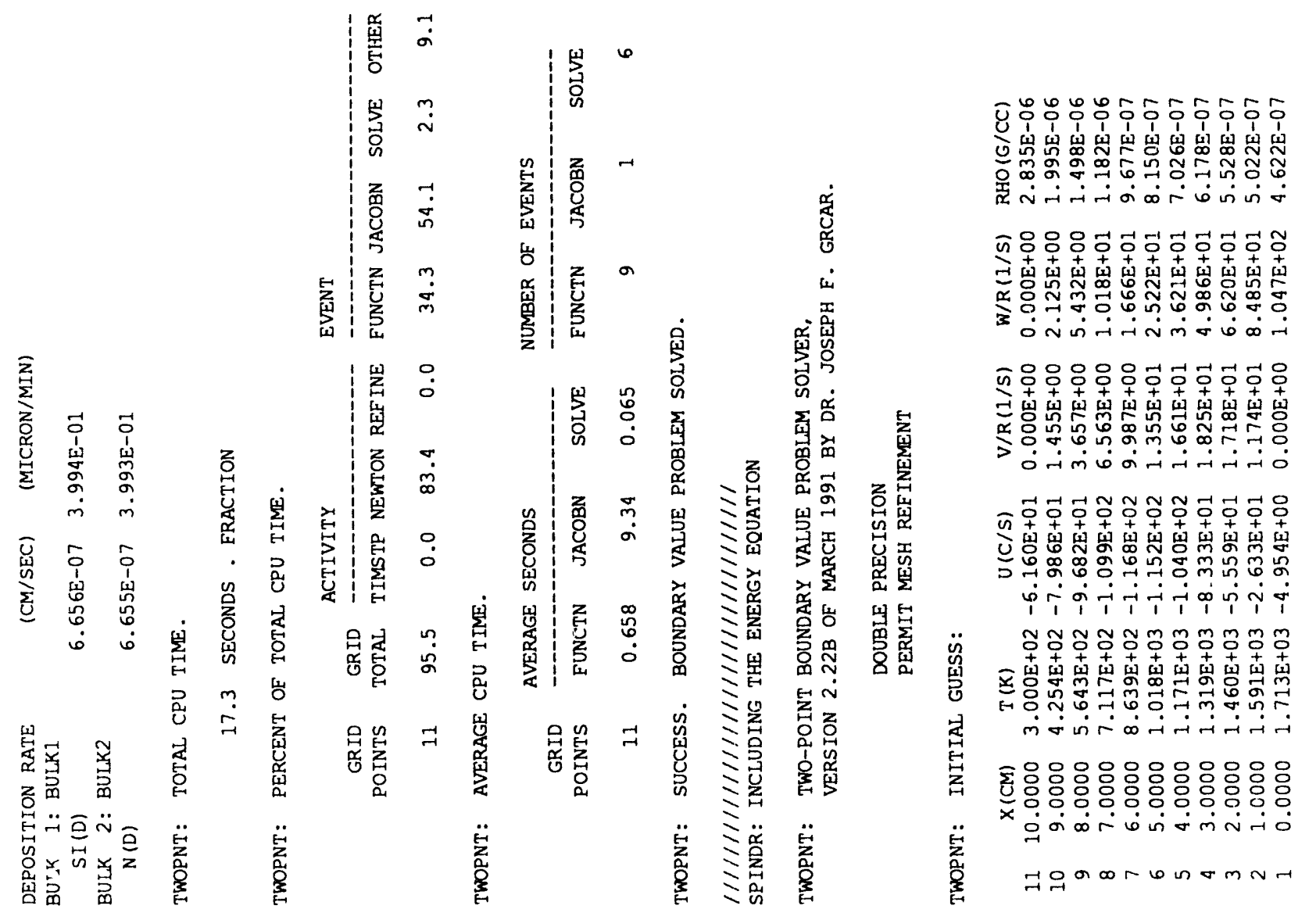




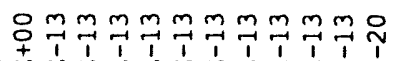

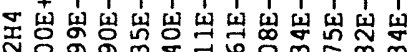

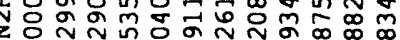

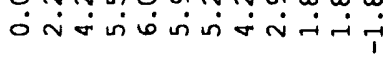
$8 \simeq \approx \simeq \simeq \approx \approx \simeq \simeq \simeq \simeq 9$

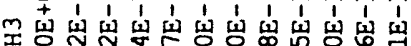

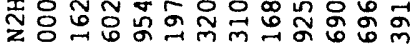

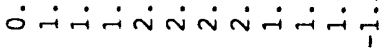
옥ㅋㅋㅋㅋㅋㅋㅋㅋㅋㅗ

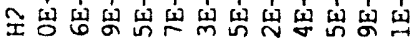

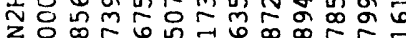

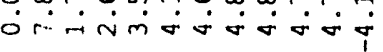

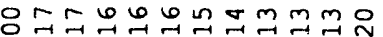

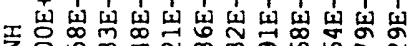

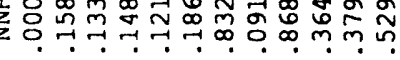
otintarintan

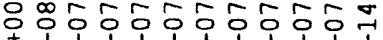

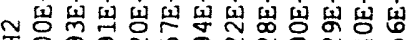

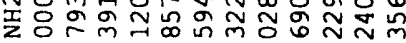

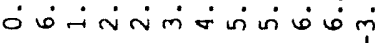

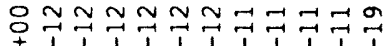

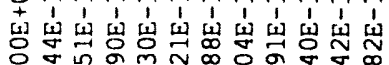

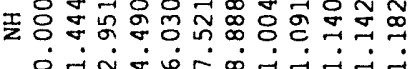
$\dot{0} \dot{\sim} \dot{\sigma} \dot{0} \dot{\sim}-\dot{i} \dot{-i}$ 8구운

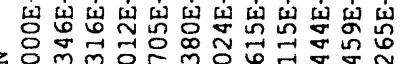

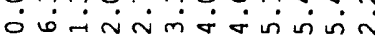

웅ㅋㅋㅋㅋㅋㅋㅋㅜ

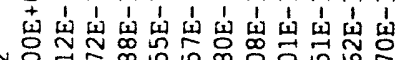
₹

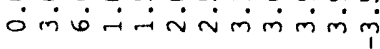

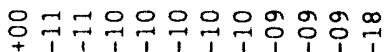

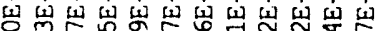

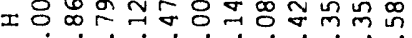
cmranmb-mm

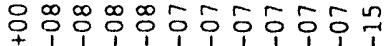

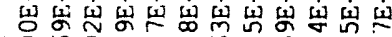

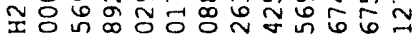

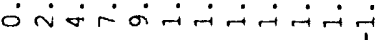

용ㅇㅇㅇㅇㅇㅇㅇㅇㅇㅇ응

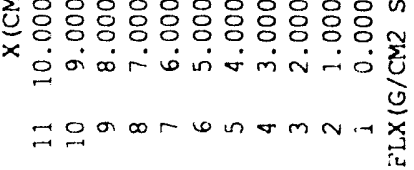

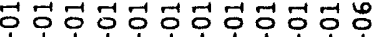

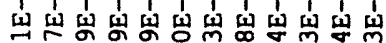

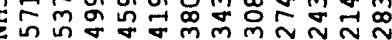
$\infty \infty \infty \infty \infty \infty \infty \infty \infty \infty)$

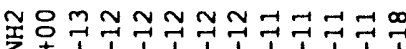

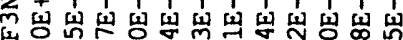

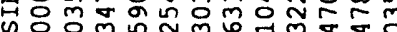
ठं

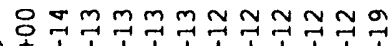

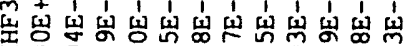

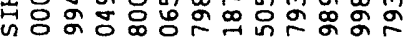

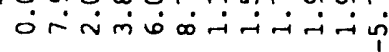

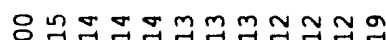

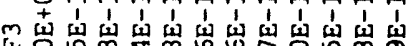

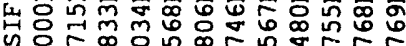

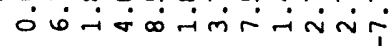

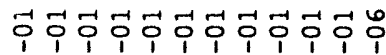

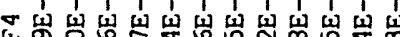

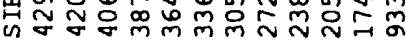

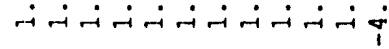

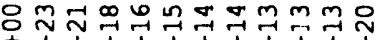

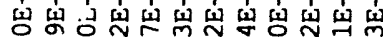

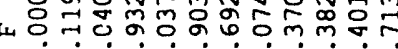
ना $\rightarrow$ tramal

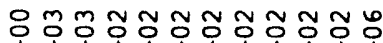

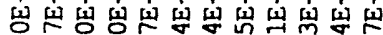

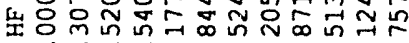
or

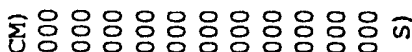

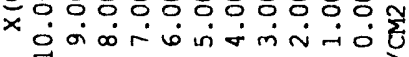

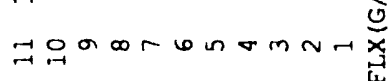

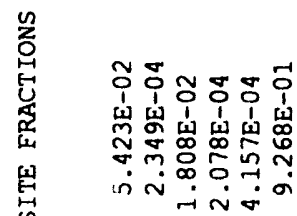

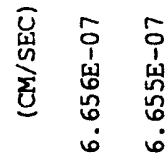

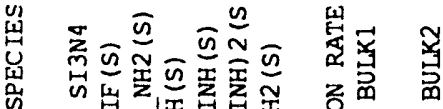

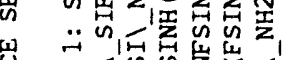
岂

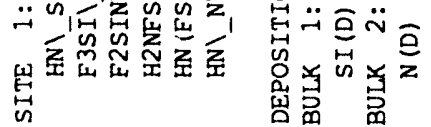




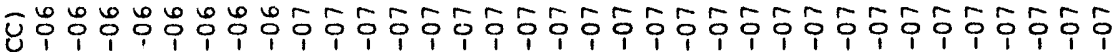

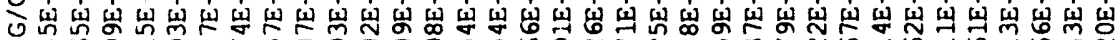

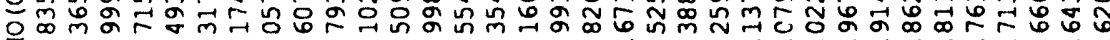
2 N N

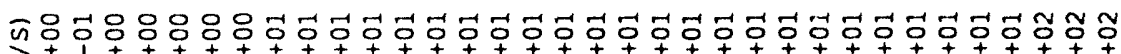

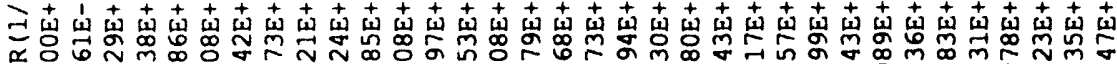

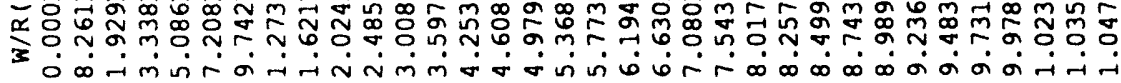

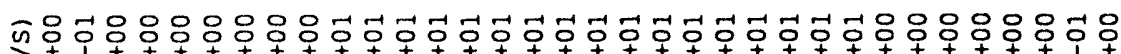

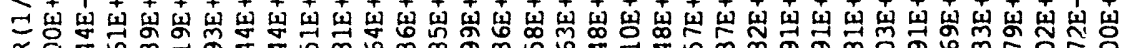

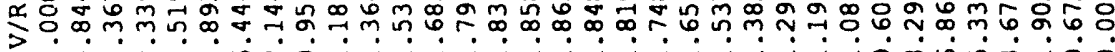

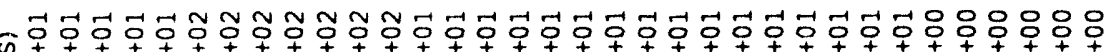

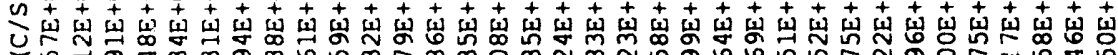

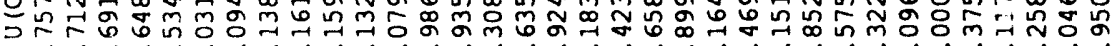

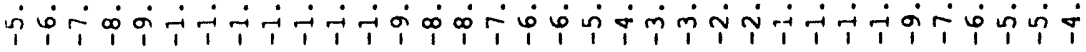

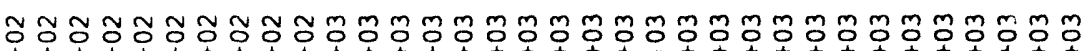

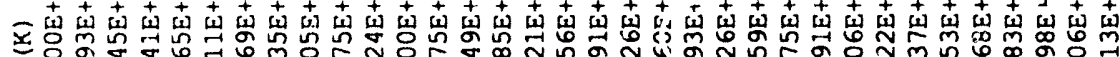

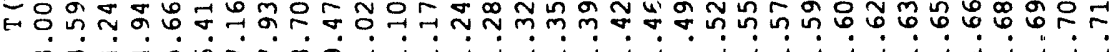

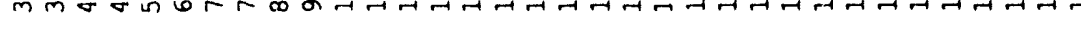

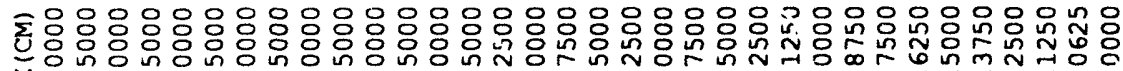
×

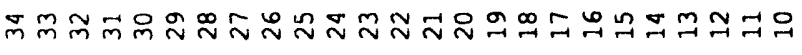

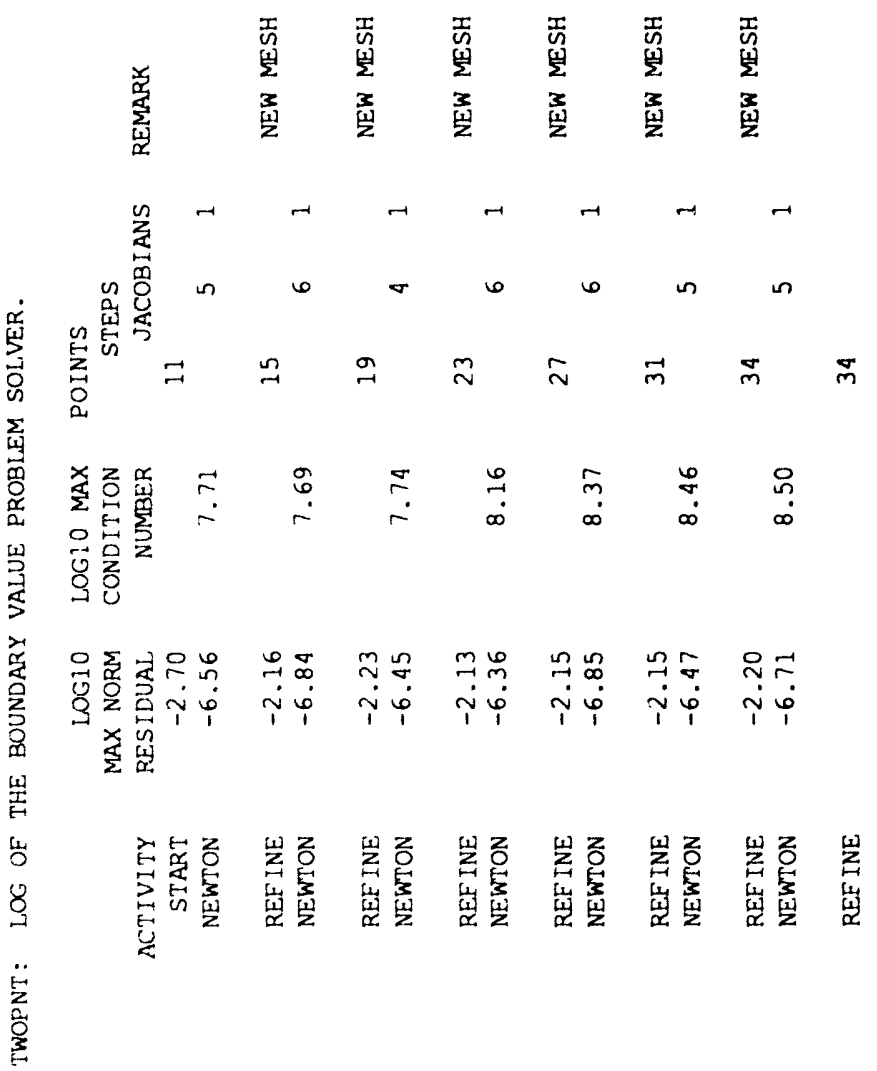




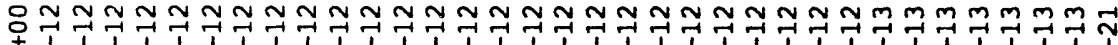

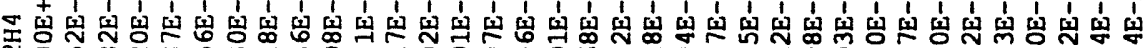
穵

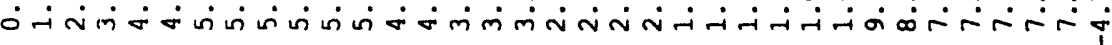

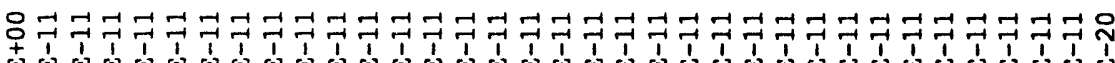

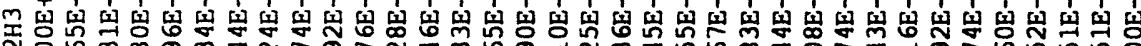
夌 8 员

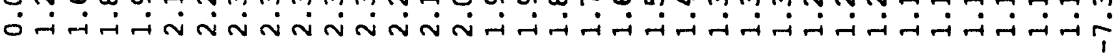

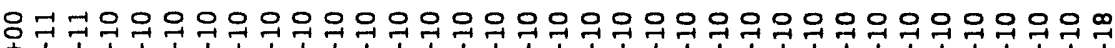

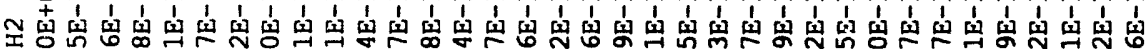

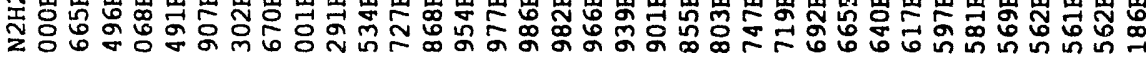
$\dot{0} \dot{\sim} \dot{\dot{H}} \dot{\sim} \dot{\sim} \dot{\sim} \dot{m} \dot{m} \dot{m} \dot{m} \dot{m} \dot{m} \dot{m} \dot{m} \dot{m} \dot{m} \dot{m} \dot{m} \dot{m} \dot{m} \dot{m} \dot{m} \dot{m} \dot{m} \dot{m} \dot{m} \dot{m} \dot{\sim}$

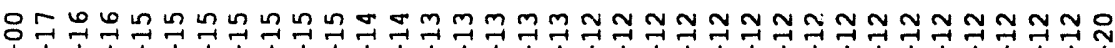

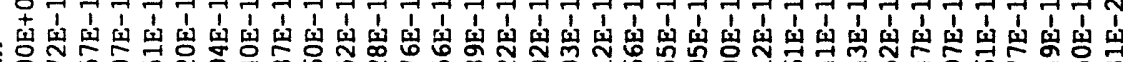

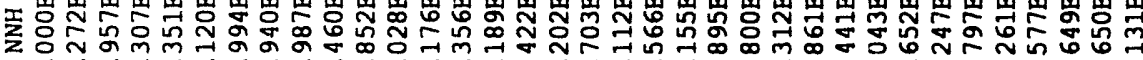

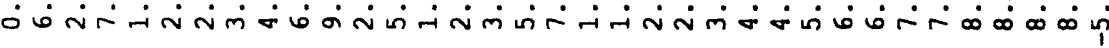

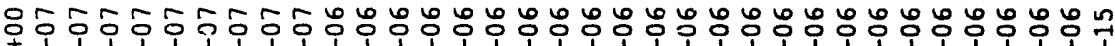

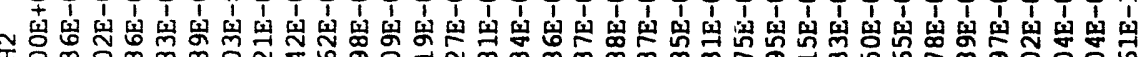

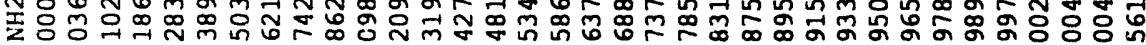

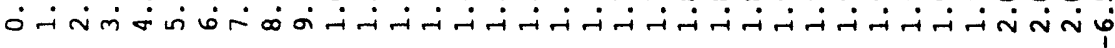

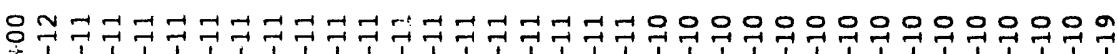
商

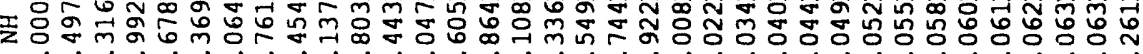

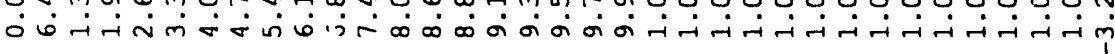

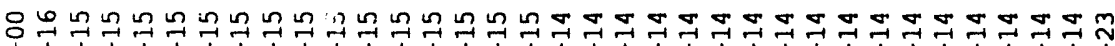

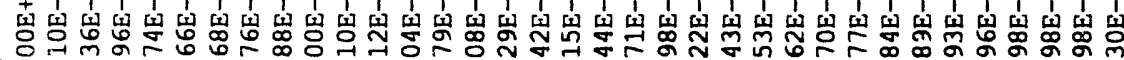

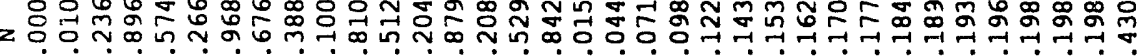

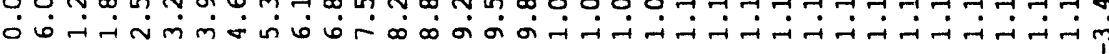

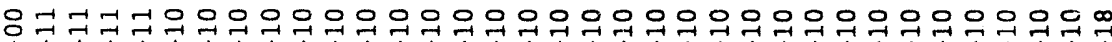

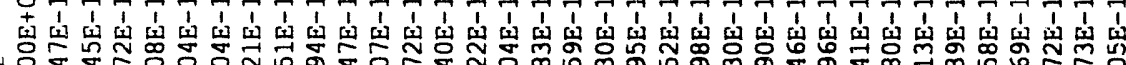
そ

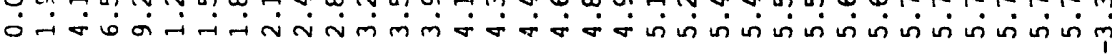

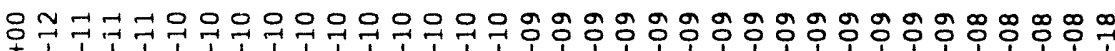

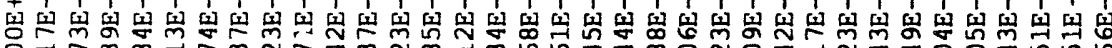
x

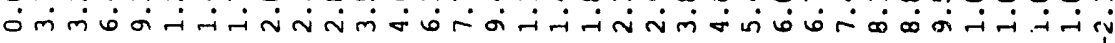

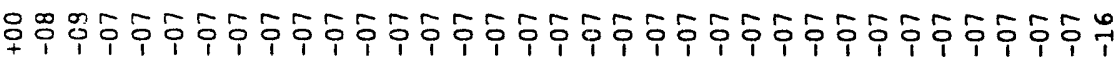

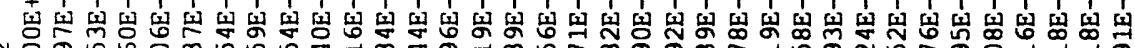

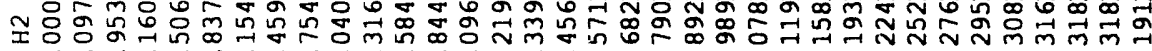

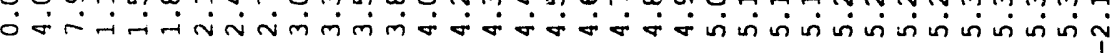

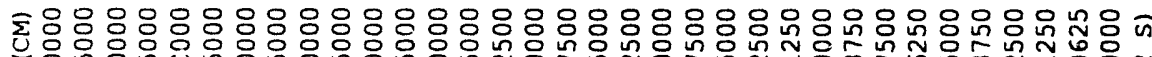

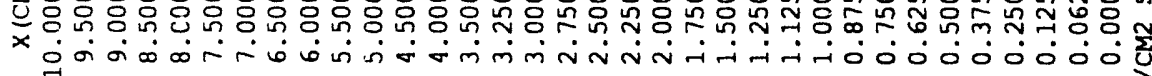

-

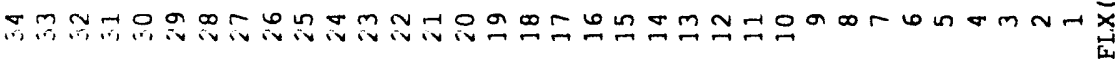

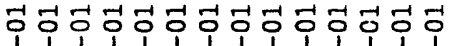

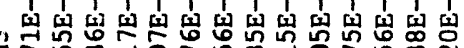

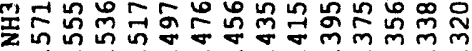
$\infty \omega_{\infty}^{\infty} \infty \dot{\infty} \infty \dot{\infty} \infty \dot{\infty} \infty \dot{\infty} \infty \dot{\infty} \infty$

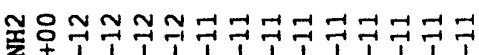

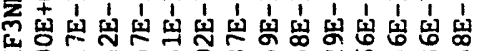

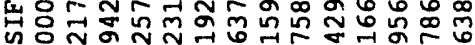

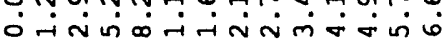

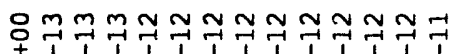

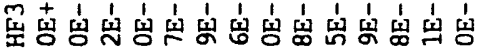

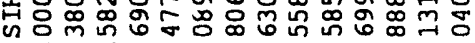

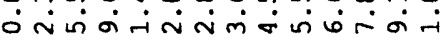

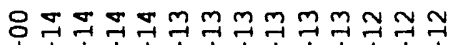

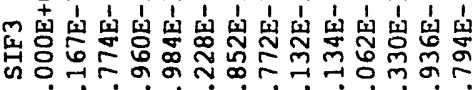

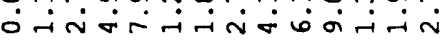

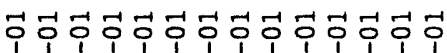

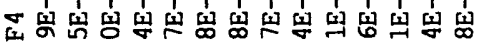

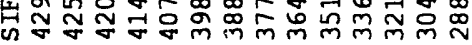

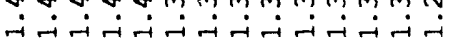

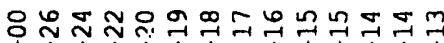

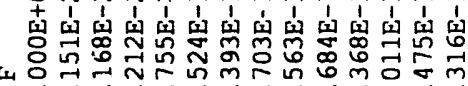

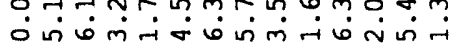

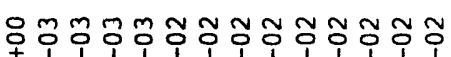

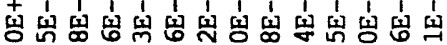

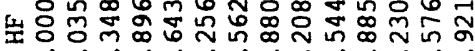

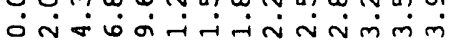

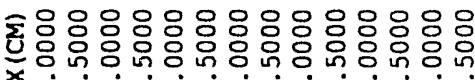
x

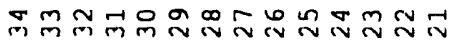




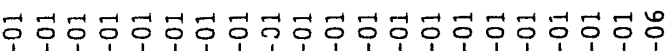

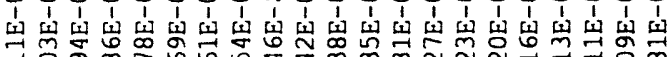

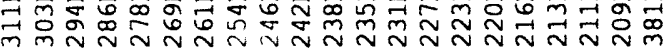

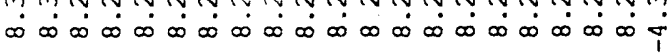

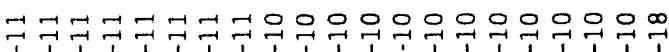

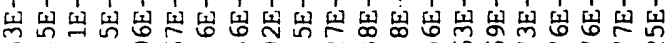

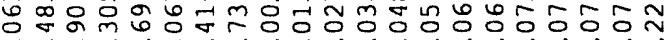

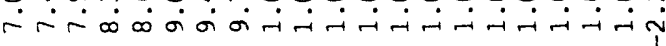

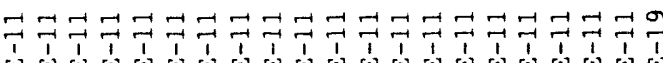

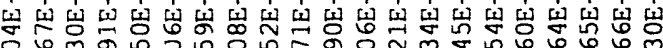

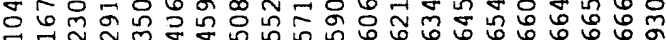

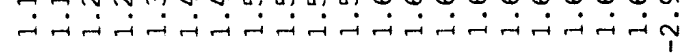

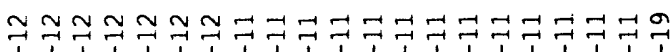

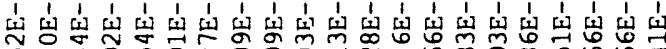

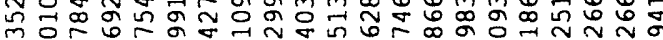

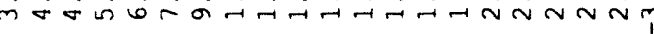

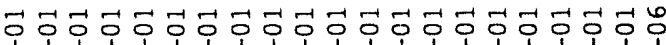

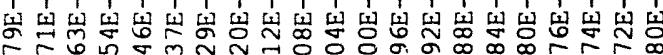

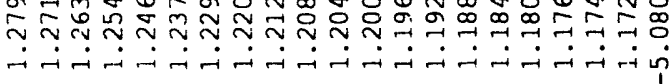

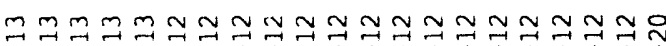

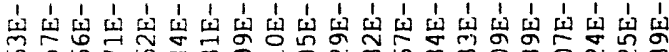
సू

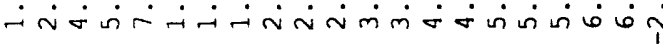

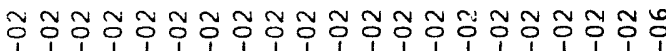

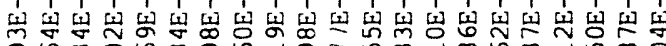

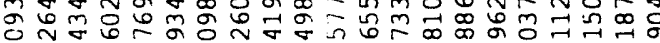

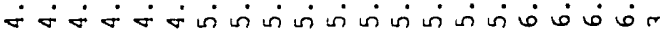

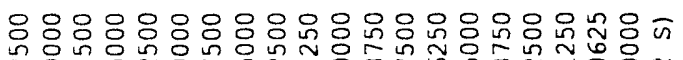

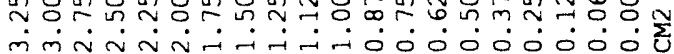

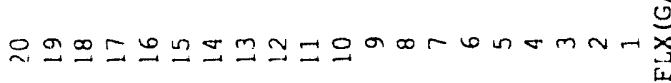

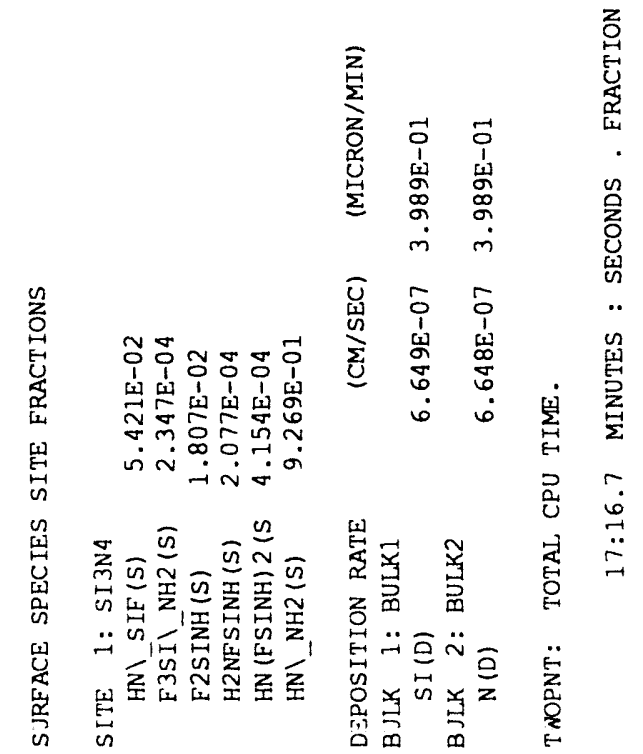




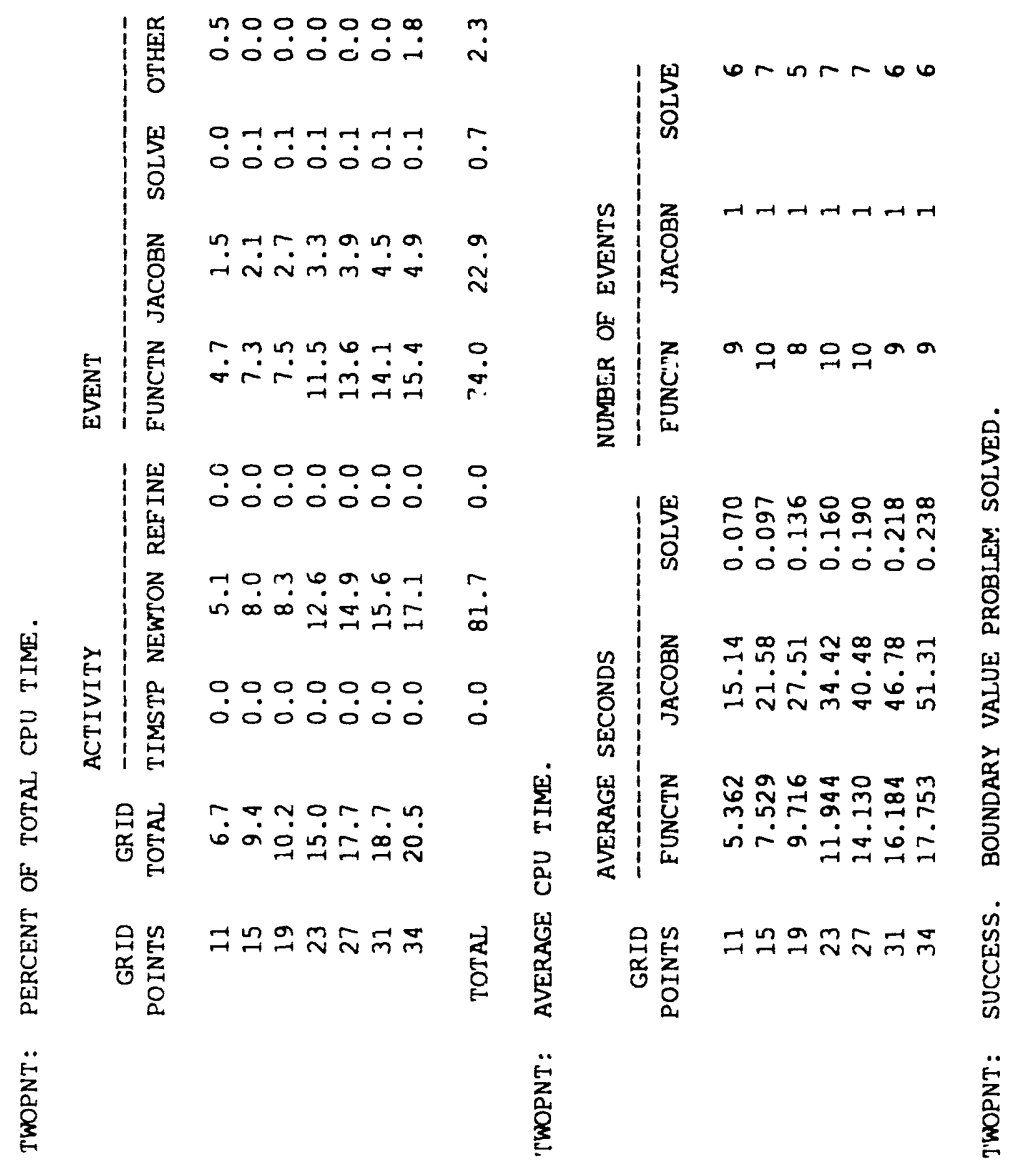




\section{REFERENCES}

1. G. Evans and R. Greif, J. Heat Trans. ASME, 109, 928 (1987).

2. D. R. Olander, I. and E. C. Fund. 6, 178 (1967).

3. D. R. Olander, I. and E. C. Fund. 6, 188 (1967).

4. M. L. Hitchman, B. J. Curtis, H. R. Brunner, and V. Eichenberger, Physicochemical Hydrodynamics, Vol. 2, edited by D. B. Spalding (Advanced Publications, London 1977).

5. R. Pollard, and J. Newman, J. Electrochem. Soc. 127, 744 (1980).

6. T. von Karman, A. Angew. Math. Mech. 1, 233 (1921). Also available as NACA-TM-1092.

7. F. M. White, Viscous Fluid Flow, (McGraw-Hill, New York 1974).

8. H. Schlichting, Boundary Layer Theory, 7th edition (McGraw-Hill, New York 1979).

9. W. G. Cochran, Proc. Cambridge Phil. Soc. 30, 365 (1934).

10. E. M. Sparrow and J. L. Gregg, J. Heat Trans. ASME 82, 294 (1960).

11. G. Evans and R. Greif, Num. Heat Transfer, submitted for publication.

12. M. E. Coltrin, R. J. Kee, and G. H. Evans, J. Electrochem. Soc. 136, 819 (1989).

13. R. J. Kee, F. M. Rupley, and J. A. Miller, "CHEMKIN-II: A Fortran Chemical Kinetics Package for the Analysis of Gas-Phase Chemical Kinetics," Sandia National Laboratories Report SAND89-8009 (1989).

14. R. J. Kee, G. Dixon-Lewis, J. Warnatz, M. E. Coltrin, and J. A. Miller, "A Fortran Computer Code Package for the Evaluation of Gas-Phase Multicomponent Transport Properties," SAND86-8246 (1986).

15. M. E. Coltrin, R. J. Kee, and F. M. Rupley, "SURFACE CHEMKIN (Version 3.7): A Fortran Package for Analyzing Heterogenous Chemical Kinetics at a Solid-Surface-Gas-Phase Interface," SAND90-8003 (1990).

16. J. F. Grcar, "The Twopnt Program for Boundary Value Problems," SAND91-8230 (1991).

17. J. F. Grcar, R. J. Kee, M. D. Smooke, and J. A. Miller, "A Hybrid Newton/Time-Integration Procedure for the Solution of Steady, Laminar, One-Dimensional, Premixed Flames," Twenty-First Symposium (International) on Combustion, The Combustion Institute, Pittsburgh, PA (1986), p. 1773.

18. J. A. Miller, R, J. Kee, and C. K. Westbrook, "Chemical Kinetics and Combustion Modeling," Annu. Rev. Phys. Chem. 41 345-387 (1990).

19. R. J. Kee, J. F. Grcar, M. D. Smooke, and J. A. Miller, "A Fortran Program for Modeling Steady Laminar One-Dimensional Premixed Flames," Sandia National Laboratories Report, SAND85-8240 (1985).

20. S. Paolucci, Sandia National Laboratories Report, SAND82-8257 (1982).

21. J. O. Hirschfelder, C. F. Curtiss, and R. B. Bird, Molecular Theory of Gases and Liquids (John Wiley and Sons, New York, 1954).

22. G. Dixon-Lewis, Proc. Royal Soc. A. 307, 111-135 (1968).

23. J. J. Dongarra, C. B. Moler, J. R. Bunch, and G. W. Stewart, "LINPACK Users' Guide," Society of Industrial and Applied Mathematics, Philadelphia (1979).

24. A. R. Curtis, M. J. Powell, and J. K. Reid, J. Inst. Math. Appl. 13, 117 (197i.1)

25. M. D. Smooke and R. M. M. Mattheij, Appl. Numer. Math. 1, 463-487 (1985).

26. R. A. Brown, L. E. Scriven, and W. J. Silliman, "Computer-Aided Analysis of Nonlinear Problems in Transport Phenomena," in Noniinear Problems in Dynamics, edited by P. Holmes, SIAM, Philadelphia (1980), p. 289.

27. H. Saito and L. E. Scriven, J. Comp. Phys. 42, 53 (1981).

28. W. E. Stewart and J. P. Sørensen "4th International/6th European Symposium on Chemical Reactor Engineering," Dechema, Frankfurt (1976). 
29. T. P. Coffee and J. M. Heimerl, Combust. Sci. and Tech. 34, 31 (1983).

30. T. P. Coffee, and J. M. Heimerl, Comb. and Flame 50, 323 (1983).

31. Y. Rueven, M. D. Smooke, and H. Rabitz, Yale University Mechanical Engineering, ME-104-85 (1985). 


\section{UNLIMITED RELEASE}

\section{INITIAL DISTRIBUTION}

1100

F. L. Vook

Attn: J. B. Gerardo, 1120

1126 W. G. Breiland

1126 M. E. Coltrin (10)

1126 P. Ho

1126 H. K. Moffat

1126 A. W. Johnson

1126 K. P. Killeen

1553 W. L. Hermina

1812 R. J. Buss

8000 J. C. Crawford

Attn: D. L. Crawford, 1900

R. J. Detry, 8200

P. L. Mattern, 8300

8244

8245

R. S. Larson

8245

D. S. Dandy

8245

G. H. Evans

8245

J. F. Grcar

8245

W. G. Houf

8245

R. J. Kee (150)

8245 E. Meeks

8245 F. M. Rupley

$8347 \quad$ W. L. Hsu

8353 C. F. Melius

8353 J. A. Miller

8361 M. D. Allendorf

8535 Publications for OSTI (10)

8535 Publications/Technical Library Processes Division (3)

3141 Technical Library Processes Division (3)

8524-2 Central Technical Files (3) 

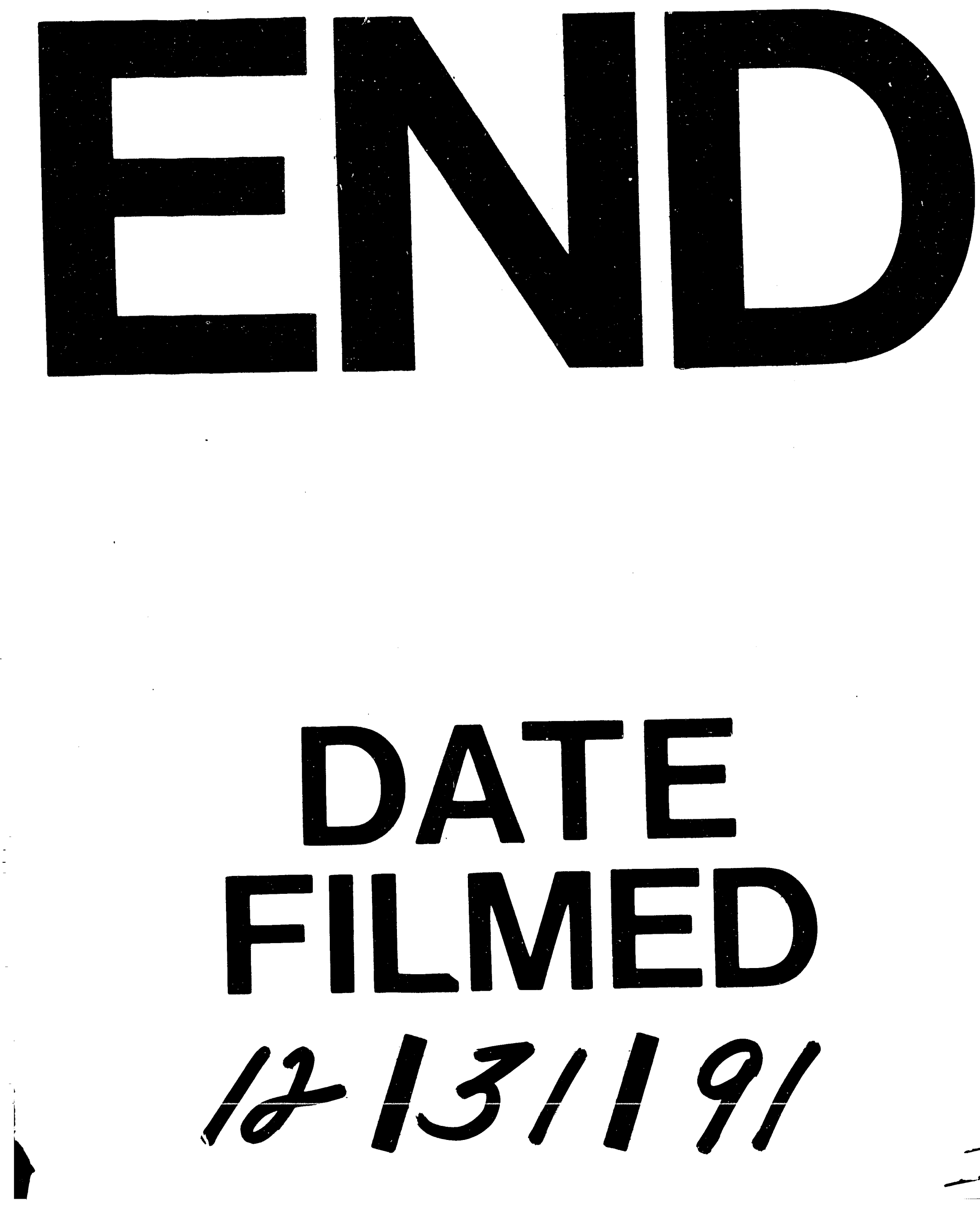
